\title{
THEORIES OF INTRAMOLECULAR VIBRATIONAL ENERGY TRANSFER
}

\section{T. UZER}

School of Physics, Georgia Institute of Technology, Atlanta, Georgia 30332-0430, USA and Bilkent University, Ankara, Turkey

with an appendix by

W.H. MILLER

Department of Chemistry, and Materials and Molecular Research Division of the Lawrence Berkeley Laboratory, University of California, Berkeley, California 94720, USA 


\title{
THEORIES OF INTRAMOLECULAR VIBRATIONAL ENERGY TRANSFER
}

\author{
T. UZER \\ School of Physics, Georgia Institute of Technology, Atlanta, Georgia 30332-0430, USA \\ and Bilkent University, Ankara, Turkey \\ with an appendix by \\ W.H. MILLER \\ Department of Chemistry, and Materials and Molecular Research Division of the Lawrence Berkeley Laboratory, \\ University of California, Berkeley, California 94720, USA \\ Editor: E.W. McDaniel Received January 1990
}

Contents:

1. Brief history and overview

2. Definition and nature of intramolecular relaxation

2.1. General molecular model for intramolecular vibrational energy redistribution (IVR)

2.2. Criteria for IVR regimes

2.3. Dephasing versus relaxation in isolated molecules

3. "Phenomenological" theories of energy sharing; approach of radiationless transitions

3.1. Molecular model for fluorescence

3.2. "Exact" molecular eigenstates

3.3. Long-time experiments

3.4. Short-time experiments

3.5. Quantum beats

4. Vibrational energy transfer in model nonlinear oscillator chains; the Fermi-Pasta-Ulam paradox and the Kolmogorov-Arnol'd-Moser theorem

4.1. The Fermi-Pasta-Ulam (FPU) paradox

4.2. The Kolmogorov-Arnol'd-Moser (KAM) theorem and nonlinear resonances
4.3. The onset of stochasticity

5. Classical microscopic theory of energy sharing; energy transfer through nonlinear resonances

5.1. Nonlinear resonances and classical mechanics of the pendulum

5.2. Vibrational energy transfer and overlapping resonances

5.3. Generalizing the pendulum

5.4. Nonstatistical effects and phase space structures

6. "Mesoscopic" description of intramolecular vibrational energy transfer

6.1. Self-consistent description of quasiharmonic mode relaxation

6.2. Relaxation of individual quasiharmonic modes and its spectral signature

Appendix. On the relation between absorption linewidths, intramolecular vibrational energy redistribution (IVR), and unimolecular decay rates $\quad 124$

References
101

\section{Abstract:}

Intramolecular vibrational energy transfer is a process central to many physical and chemical phenomena in molecules. Here, various theories describing the process are summarized with a special emphasis on nonlinear resonances. A large bibliography supplements the text.

Single orders for this issue

PHYSICS REPORTS (Review Section of Physics Letters) 199, No. 2 (1991) 73-146.

Copies of this issue may be obtained at the price given below. All orders should be sent directly to the Publisher. Orders must be accompanied by check.

Single issue price Dfi. 56.00 , postage included 


\section{Brief history and overview}

The flow of vibrational energy in excited molecules is central to chemical and molecular dynamics. Various new developments in physics, chemistry and technology of the 1970's and 80's have tended to converge in intramolecular energy transfer (also known by the acronym IVR, for intramolecular vibrational energy redistribution): the increasingly powerful and accurate methods of laser excitation and analysis of molecular processes on the experimental side, and the burgeoning interest in the classical-mechanical and quantal behavior of nonlinear oscillator systems on the theoretical side (for extensive reviews, see Chirikov [1979], Zaslavsky [1981, 1985], Sagdeev et al. [1988], Eckhardt [1988]). Since its inception, intramolecular energy transfer has been a field driven by experiment. This is a natural outcome of the usually overwhelming complexity of intramolecular interactions. Even a superficial survey of the theoretical literature of the last thirty or so years reveals a few strands of theoretical thinking on IVR, each of which was suggested to some degree by the experimental achievements at the time.

While the experimental situation has been reviewed many times (e.g., Parmenter [1982, 1983], Smalley [1982], Bondybey [1984]), it seems that the various theoretical views of the process have not been collected in one place before. This is the modest aim of this overview. During the "narration" I will occasionally refer to experiments, but these references are not intended to be comprehensive because of space restrictions. Therefore, some slight to experimental accomplishments is inevitable and I apologize for it at the outset.

Historically the most widely known studies of intramolecular vibrational redistribution are associated with thermal unimolecular reactions [Robinson and Holbrook 1972; Forst 1973; Pritchard 1984]. The currently accepted varieties of unimolecular reaction rate theories arose through the testing of Slater's dynamical theory [Slater 1959] and the statistical Rice-Ramsperger-Kassel-Marcus (RRKM) theories [Marcus 1952]. The key contention in this debate was the very existence of intramolecular energy transfer. Slater's theory pictured the excited molecule as an assembly of harmonic oscillators. Within the Slater framework, vibrational energy sharing between modes is forbidden, and the unimolecular reaction occurs when a reaction progress variable, the so-called "reaction coordinate" reaches a critical extension by the superposition of various harmonic mode displacements. In contrast, the RRKM theory assumes that excitation energy randomizes rapidly compared to the reaction rate, and is distributed statistically among the modes prior to reaction. (A similar formalism, albeit for the decay of excited nuclei, had been introduced by Bohr and Wheeler [1939] independently from the development of the statistical theories in chemical physics). The assumption of randomization, which turns out to be widely valid, was needed very early in the theoretical development because very little was known about energy sharing in molecules at the time.

The statistical approach reduces the (usually very high) dimensionality of the molecular problem in a manner similar to traditional "transition state theory" [Glasstone et al. 1941]. The phase-space motion of the system along the reaction coordinate is assumed to be separable from its motion in all other possible modes, at least in the vicinity of a multidimensional surface in phase space that separates reagents from products. This latter construct is called the "dividing surface" (for reviews, see Hase [1976], Truhlar et al. [1983], Hase [1983]), and the reaction rate calculation is thus reduced to calculating the rate of passage of systems across the dividing surface, a "bottleneck" in one dimension only.

The first demonstration that IVR is a real physical phenomenon (Butler and Kistiakowski [1960]) concerned molecules in the ground electronic state with the high vibrational excitation characteristic of 
reacting species. The kinetic and spectroscopic evidence for intramolecular relaxation processes in polyatomic molecules has been discussed critically by Quack [1983], who concluded that, on the basis of their 0.1-10 ps timescale, such processes are slow compared with vibrational periods but fast compared with reactive and optical processes. This picosecond time scale turns out to be typical (see Kulp et al. [1985], Bagratashvili et al. [1986]). The "chemical timing" experiments of the Parmenter group (for an overview, see Parmenter [1983]) as well as the experiments of Felker and Zewail [1984, 1985a-d] reveal the IVR process by providing striking images of its temporal progress.

Detailed experimental studies performed in the early 1960's (reviewed by Oref and Rabinovitch [1979]) produced results in harmony with the statistical theory. The wide success of the predictions of RRKM theory have been taken as proof of vibrational energy randomization. However, these early experiments are not of a dynamical nature. Direct observations require a dynamical experiment in which a very well specified initial state (or a superposition of states) is prepared. This state decays and a measurement is made at a later time to demonstrate that the system is in a state which differs from the initial one.

The nature of the initial state in many of these early experiments is a statistical superposition because of the collisional preparation of the initial state. Could it be the statistical preparation of the initial state which gives such good agreement with experiments? In fact, the RRKM theory has been reformulated by assuming that the vibrational relaxation is slow on the scales of molecular decomposition [Freed 1979]. These two formulations of the statistical theory take different viewpoints about the meaning of IVR. The randomizing approach considers the vibrational states in terms of a zeroth-order harmonic description, whereas the nonstatistical theory considers the vibrational eigenstates which diagonalize the full molecular Hamiltonian. The question arises, then: what is the proper description of the initial state?

At about the same time as the last reformulation of the statistical theory by Marcus [1952], a calculation was being performed by Fermi, Pasta and Ulam [1955] which was to inspire physicists to rethink their ideas on energy relaxation in coupled oscillator systems. The purpose of this calculation was to see, in detail, the approach to ergodicity in a chain of coupled nonlinear oscillators. The result, which has become known as the Fermi-Pasta-Ulam (FPU) paradox, surprised researchers by showing very little relaxation, thus demonstrating that dynamics of energy sharing even in a simple coupled oscillator system can be far from straightforward and need not be statistical at all. This disturbing lack of ergodicity was explained by Ford [1961] by showing that the FPU system lacked an essential ingredient for energy sharing, namely active nonlinear resonances. Also known as "internal" or "Fermi" resonances, these integer ratios between frequencies are the unifying theme of this overview. The by then extant but little known Kolmogorov-Arnol'd-Moser (KAM) theorem revealed the connection between these resonances and ergodicity (for reviews, see Ford [1975], Berry [1978]).

These twin developments of the fifties had a considerable impact on IVR research. While, on the one hand, statistical theory affirmed the existence of redistribution for real molecules and the conclusions of experiments were in harmony with statistical assumptions, on the other hand paradigmatic, simple coupled oscillator systems were sometimes found to relax nonstatistically, and sometimes not at all. In this context it is worth noting that statistical theories of chemical reactions have always allowed for the presence of nonstatistical effects, by, for instance, excluding certain "inactive" modes from consideration because they are poorly coupled to the rest of the molecule [Robinson and Holbrook 1972; Forst 1973]. Notwithstanding the many couplings and resonances in even small molecules, the possible existence of FPU-like behavior in molecular systems has been an intriguing and persistent question, and one that will be addressed in this overview. 
The plan of this article is as follows. After general remarks about the nature of IVR, the quantum process is described in sections 2 and 3 by making relatively few assumptions about the detailed coupling and energy transfer dynamics. This statistical-mechanical description is based on the theory of radiationless transitions, where the central issue is to connect molecular energy-level structure to the dynamics of energy flow [Hunt et al. 1962; Robinson and Frosch 1962; Byrne et al. 1965; Robinson 1967; Bixon and Jortner 1968, 1969a, b]. The "intermediate" version of this theory [Lahmani et al. 1974; Freed 1976; Freed and Nitzan 1980] turns out to be applicable to common experimental scenarios. We shall refer to this description as the "phenomenological" theory, to indicate its frequent use of averaged (over a large number of states) properties which can be determined from experimental data (e.g., Matsumoto et al. [1983]). Section 2 refers specifically to IVR in a single Born-Oppenheimer electronic state, whereas in section 3 the emphasis is on molecular fluorescence as a well-established diagnostic of IVR.

A contrast to this macroscopic approach shall be demonstrated in section 4 when we discuss the place of the FPU paradox and the KAM theory in the development of the field. This section will bring to the fore the central role nonlinear resonances play in intramolecular energy sharing. Subsequently, in section 5, we focus on energy transfer through isolated and interacting nonlinear resonances [Oxtoby and Rice 1976; Kay 1980; Jaffé and Brumer 1980; Sibert et al. 1982a,b; Sibert et al. 1984a,b], where the individual couplings are examined rather than representative average couplings. In that section, we also summarize the treatment of long-time correlations in intramolecular dynamics by means of "bottlenecks" in phase space [Davis 1985]. In section 6, we review work that connects explicitly nonlinear oscillations to the experimentally accessible manifestations of IVR [Kuzmin et al. 1986a; Stuchebryukhov 1986] using density matrix techniques [Faid and Fox 1987, 1988]. The appendix examines the connection between absorption line widths, IVR and unimolecular decay rates.

There are many fundamental and exciting developments in theoretical physics and chemistry which are intimately connected with the IVR problem. Restrictions of space preclude their thorough discussion and the explicit tracing of the connections. Among these are: mode specific chemistry [Thiele et al. 1980a,b; Bloembergen and Zewail 1984; Miller 1987]; (for recent experimental examples, see Butler et al. [1986a, 1987]); quantum transition state theory [Tromp and Miller 1986], photodissociation [Simons 1984; Brumer and Shapiro 1985]; electronic relaxation [Boeglin et al. 1983; Amirav and Jortner 1987], chemiluminescent processes and ultrafast energy transfer among electronically excited states [Gelbart and Freed 1973; Bogdanov 1980; Gole 1985], and radiationless processes in general [Freed 1976; Ranfagni et al. 1984]; and the vast subject of unimolecular and intramolecular dynamics [Hase 1976; Kay 1976, 1978; Brumer and Shapiro 1980; Heller 1980; Hase 1981; Marcus 1983; Heller 1983; Peres 1984; Stechel and Heller 1984; Marcus 1988; Huber and Heller 1988; Huber et al. 1988; Ito 1988], and its relation to chaos [Kay 1983; Stechel and Heller 1984; Ramachandran and Kay 1985; Farantos and Tennyson 1987; Kay and Ramachandran 1988; Shapiro et al. 1988], as well as localization [Kay 1980; Heller 1987; O'Connor and Heller 1988; Dumont and Pechukas 1988; Parris and Phillips 1988], and the spectral signature of chaos [Dai et al. 1985a,b; Hamilton et al. 1986; Pique et al. 1988; Lombardi et al. 1988; Xie 1988]. For a "super-review" that includes most intramolecular processes, see Jortner and Levine [1981]. I must also leave out energy transfer on surfaces [Tully 1985; Micklavc 1987; Gadzuk 1987, 1988; Zangwill 1988] in spite of significant developments in the microscopic probing of vibrational energy transfer between absorbates and surfaces [Heidberg et al. 1985; Jedrzejek 1985; Heidberg et al. 1987; Heilweil et al. 1988a,b; Harris and Levinos 1989]. 


\section{Definition and nature of intramolecular relaxation}

Collisional relaxation of polyatomic molecules in a thermal environment is a well-defined process (among standard reviews are Quack and Troe [1977], Kneba and Wolfrum [1980]; for some new developments emphasizing the connection to the intramolecular relaxation process, see Rice and Cerjan [1983], Lawrance and Knight [1983], Villalonga and Micha [1983], Nalewajski and Wyatt [1983, 1984], Gilbert [1984], Lim and Gilbert [1986], Orr and Smith [1987], Haub and Orr [1987], Koshi et al. [1987], Kable and Knight [1987], Bruehl and Schatz [1988], D.J. Muller et al. [1988], Kable et al. [1988], Rainbird et al. [1988], Rock et al. [1988], Gordon [1988], Parson [1989, 1990]; for competition between inter- and intramolecular energy transfer, see Straub and Berne [1986]). The same cannot be said about the intramolecular vibrational relaxation of isolated molecules. A process can be considered intramolecular if there is negligible collisional and radiative interaction with the environment during the time scale of interest, which is less than a nanosecond. Vibrational relaxation can be defined as irreversible decay (on the time scale of interest) of a localized vibrational excitation in a molecule [Quack 1983].

Dynamical processes in isolated molecules are governed by the time-dependent Schrödinger equation

$$
H \Psi=\left(H_{0}+W\right) \Psi=\mathrm{i} \hbar \partial \Psi / \partial t
$$

In this section, we will take $\hbar=1$. Neglecting radiative decay (or working in a basis that includes the states of the radiation field) the solutions of (2.1) are oscillatory. Assuming that eq. (2.1) is rewritten in terms of the amplitudes of the eigenstates of $H_{0}$ (the "unperturbed", diagonal part of $H$ ), and that the coupling $W$ is off-diagonal in this basis,

$$
\mathrm{i}^{\prime}=\left(\boldsymbol{H}_{0}+\boldsymbol{W}\right) \boldsymbol{b}
$$

For a time-independent $H$, the solution of (2.2) can be written as

$$
\begin{aligned}
& \boldsymbol{b}(t)=\boldsymbol{U}\left(t, t_{0}\right) \boldsymbol{b}\left(t_{0}\right), \\
& \boldsymbol{U}\left(t, t_{0}\right)=\exp \left[-\mathrm{i} \boldsymbol{H}\left(t-t_{0}\right)\right]
\end{aligned}
$$

The time-evolution matrix solves the Liouville-von Neumann equation for the time-dependent density matrix $\boldsymbol{\sigma}$,

$$
\boldsymbol{\sigma}(t)=\boldsymbol{U}\left(t, t_{0}\right) \boldsymbol{\sigma}\left(t_{0}\right) \boldsymbol{U}^{+}\left(t, t_{0}\right)
$$

as well as the Heisenberg equation of motion for the representations of any observable, $Q$ (in particular the coordinates and momenta of the atoms),

$$
Q(t)=U^{+}\left(t, t_{0}\right) Q\left(t_{0}\right) U\left(t, t_{0}\right)
$$

When $H$ is known, eqs. (2.3)-(2.6) constitute the most general solution of a well-defined mathematical formulation of the physical problem of intramolecular motion. While the solution is straightforward in principle, both the size of the matrices and the possible intricacies of the couplings make the 
emergence of a relaxation from these equations somewhat subtle. The very large size of these coupled equations have naturally invited many ingenious approximation schemes (like those of Bixon and Jortner [1968], Lahmani et al. [1974], Kay [1974, 1976, 1978], Kay et al. [1981], Milonni et al. [1983], and Nadler and Marcus [1987]). By using appropriate reduced Hamiltonians, the number of equations crucial for the time development of nonstationary states can be decreased (see Mukamel [1981], and also the "adiabatically reduced equations" method due to Voth and Marcus [1986], Klippenstein et al. [1986], and Voth [1987]). The use of artificial intelligence methods for IVR problems has been advocated by Lederman et al. [1988], and Lederman and Marcus [1988]. For other procedures, see Heller [1981a,b], Scheck and Wyatt [1987] and Lopez et al. [1988]. Grad et al. [1987] have used a semiclassical reduction procedure. Classical and semiclassical aspects of the intramolecular relaxation process have been examined by Jaffé and Brumer [1984, 1985], Parson and Heller [1986a,b] and Parson [1988].

An interesting way of seeing the emergence of relaxation in a system that contains only infinitely long-lived eigenstates is to consider "coarse-grained", i.e., averaged or reduced quantities [Zwanzig 1960; Mori 1965; Kay 1974, 1976, 1978; Nordholm and Zwanzig 1975; Garcia-Colin and del Rio 1977; Ramaswamy et al. 1978; Alhassid and Levine 1979; Kay et al. 1981; Quack 1978; 1981a, 1983; Lupo and Quack 1987]. These are quantities that do not contain full mathematical information about the system; usually simpler mathematical structures arise from coarse-grained quantities. In what follows, we will use the argument and notation of Quack [1981a, 1983] who concentrates on coarse-grained level populations $p_{K}$ for approximately isoenergetic states characterized by the same quantum number $K$ in a particular local vibration (i.e., separable in $H_{0}$ ) but different quantum numbers for other degrees of freedom,

$$
p_{K}=\sum_{k}^{\prime} p_{k(K)}=\sum_{k=x+1}^{x+N} b_{k(K)} b_{k(K)}^{*} .
$$

Here $\Sigma^{\prime}$ indicates sums over the states in one level, capital letters being reserved for levels, and $x$ denotes states which are not included in the summation. Using eqs. (2.3) and (2.4), one obtains

$$
p_{K}(t)=\sum_{k}^{\prime} \sum_{J} \sum_{j}^{\prime}\left|U_{k j}\right|^{2}\left|b_{j}(0)\right|^{2}+\sum_{k} \sum_{l} \sum_{j \neq l} U_{k j} U_{k l}^{*} b_{j}(0) b_{l}^{*}(0)
$$

The second sum vanishes if the terms in it have uncorrelated phases, leaving the coarse-grained level population as

$$
p_{K}(t)=\sum_{J} \sum_{j}^{\prime} \sum_{k}^{\prime}\left|U_{k j}\right|^{2} p_{j}(0)
$$

If only the coarse-grained level populations $P_{J}=\Sigma_{j}^{\prime} P_{j}$ are measurable, the same "random phase" argument as before allows

$$
P_{K}(t)=\sum_{J} P_{J}(0) Y_{K J}(t), \quad Y_{K J}(t)=N_{J}^{-1} \sum_{j}^{\prime} \sum_{k}^{\prime}\left|U_{k j}\right|^{2}
$$

where $N_{J}$ is the number of states in one level. This matrix $\boldsymbol{Y}$, under fairly general conditions, can be expressed [Quack 1979a] as an exponential involving a time-independent matrix $\boldsymbol{K}$ [Quack 1978],

$$
\boldsymbol{Y}(t)=\exp \left[\boldsymbol{K}\left(t-t_{0}\right)\right]
$$


and $\boldsymbol{p}(t)=\boldsymbol{Y}(t) \boldsymbol{p}(0)$. This can be reduced [Quack 1978, 1981a] to the differential form of the Pauli master equation [Pauli 1928],

$$
\dot{P}=K p
$$

In contrast to the oscillatory solutions of eqs. (2.1)-(2.4), these equations, (2.11) and (2.12), have decaying solutions, denoting relaxation. For sufficiently short times the exponential function can be expanded and one obtains [Quack 1978]

$$
\boldsymbol{Y}(t)=\boldsymbol{1}+\boldsymbol{K}\left(t-t_{0}\right)+\cdots, \quad \boldsymbol{K}_{K J}=2 \pi\left|\boldsymbol{W}_{K J}\right|^{2} / \Delta_{K},
$$

where $\left|\boldsymbol{W}_{K J}\right|^{2}$ stands for the average square coupling element between the states in levels $K$ and $J$, and $\Delta_{K}$ is the average frequency separation of states in level $K\left(1 / \Delta_{K}\right.$ needs to be replaced by the density $\rho_{k}$ in the case of the continuum). The preceding development makes clear the factors that need to be considered carefully when studying intramolecular relaxation: the definition of what is being observed (e.g., what is relaxing into what), the time scale over which the observation is taking place, which Hamiltonian and which basis set is being used for the description. Moreover, the preceding development is valuable in connecting Golden-Rule type of first-order perturbation theory prescriptions, which are widely used in IVR, to a fundamental view of the process [Voth 1987].

Of course, intramolecular relaxation is a more general phenomenon than the somewhat restricted Pauli master equation would suggest, i.e., there are selections of course-graining which make oscillatory behavior possible [Quack 1981a], which are, however, the exception rather than the rule. We will see examples of such exponential behavior as well as coherences in the following sections. The Pauli master equation is merely one (and the earliest) attempt to describe relaxation phenomena in a quantummechanical setting [Van Hove 1962]; it proceeds by reducing the full density-matrix equation to a system of coupled equations for the diagonal density-matrix elements alone. The derivation, as well as the set of companion equations for the off-diagonal matrix elements, the "coherences", have been critically studied by Fox [1978, 1989]. The effect of these coherences proves valuable, e.g., in the theory of spectral lineshapes [Faid and Fox 1988].

\subsection{General molecular model for intramolecular vibrational energy redistribution (IVR)}

Figure 1 shows schematically the vibrational energy levels in a polyatomic molecule relevant to intramolecular energy transfer, as described lucidly by Freed [1981], whose notation will be adopted. The entire level in this figure belongs to either an excited electronic state (experiments reviewed by Smalley [1982], Parmenter [1982, 1983]) or the ground electronic state (e.g., Kim et al. [1987], and overtone experiments reviewed by Crim [1984, 1987]). Here, $\phi_{0}$ represents a vibrational level that carries dipole oscillator strength to some excited zero-order vibrational level $\phi_{s}$. These zero-order levels may be local modes, or normal modes chosen for reasons of convenience, or a combination thereof (e.g., Quack [1981b], Lederman et al. [1983], Child and Halonen [1984], Quack [1985], Persch et al. [1988]). The photophysical experiment begins with the molecule being in some thermally accessible vibrational-rotational level.

Isoenergetic with the low-lying levels of $\phi_{s}$ is a dense manifold of vibrational levels which do not carry oscillator strength from the ground vibrational level $\phi_{s}$ or any of the other thermally accessible vibrational levels: dipole-allowed transitions between thermally accessible ground-state vibrational 


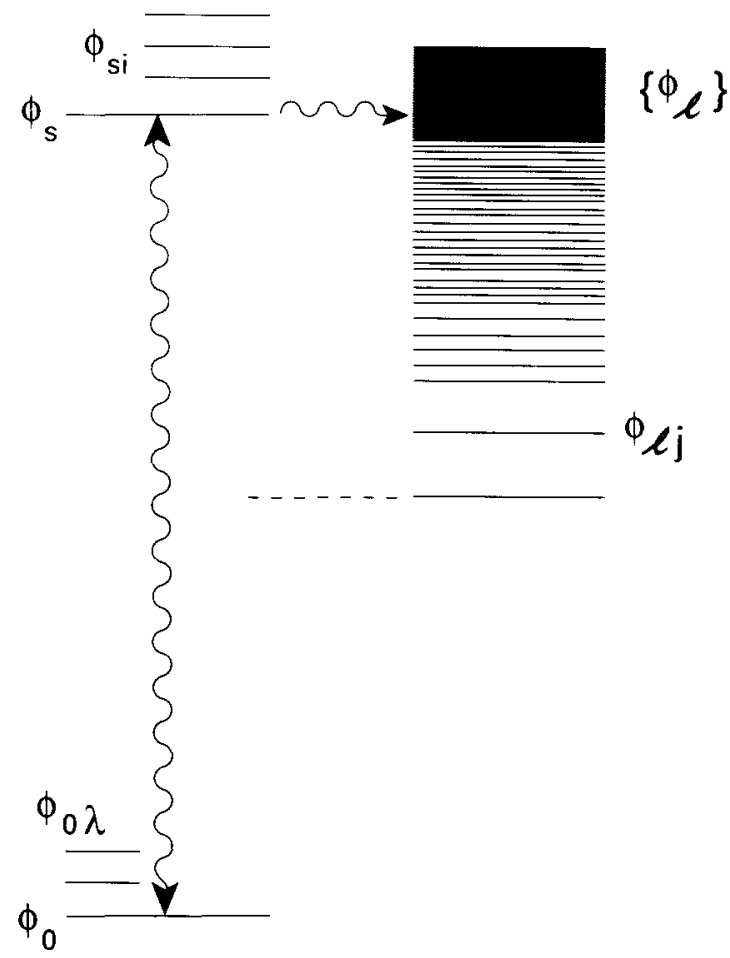

Fig. 1. Molecular energy level diagram used to discuss radiationless processes in polyatomic molecules. $\phi_{0}$ is the ground electronic state, and $\phi_{0 \lambda}$ denotes a thermally accessible component of this state. Transitions from $\phi_{0 \lambda}$ to the state $\phi_{s}$ are allowed while those to $\left\{\phi_{l}\right\}$ are forbidden. $\phi_{s i}$ designates a component of $\phi_{s}$, and $\phi_{l j}$ is a component of $\phi_{l}$. (Adapted from Freed [1981]).

levels, $\phi_{0 \lambda}$, and these high-lying states $\left\{\phi_{l}\right\}$ are not possible due to either unfavorable Franck-Condon factors or because of symmetry considerations. The zeroth-order functions $\left\{\phi_{s}, \phi_{l}\right\}$ are not exact eigenfunctions of the molecular vibrational Hamiltonian because of the presence of anharmonicities and Coriolis couplings in the normal-mode description, or, if a local mode description is used, because of local mode-local mode couplings and anharmonicities.

While the zero-order eigenstates based on the separability of vibrations leads one to expect a sharp absorption spectrum peaked at the energies of $\phi_{s}$, the presence of $\left\{\phi_{l}\right\}$ alters this situation. The alterations depend partly on the density of levels in this manifold, $\rho_{l}$. When the manifold $\left\{\phi_{l}\right\}$ is sparse (as might be expected in small molecules), perturbations in the spectra may arise due to the presence of the manifold. This small-molecule limit of low densities is an ideal scenario for the assignments and analysis of spectral lines.

When, on the other hand, the manifold $\left\{\phi_{l}\right\}$ is dense, it can behave like a continuum on the timescale of the experiment, and produce irreversible relaxation from $\phi_{s}$ to $\left\{\phi_{l}\right\}$. Between these extremes lies an intermediate case which displays some characteristics of both the small and large molecule limits because of either strong variations in the $\phi_{s}-\left\{\phi_{l}\right\}$ couplings (due to symmetry considerations, say) or when the density $\rho_{l}$ is too high to allow the resolution or the assignment of individual levels but still not high enough to lead to irreversible relaxation. The intermediate case has been termed the "too-many-level small-molecule case" [Freed 1981] and is widely encountered in current experiments, as we will see in detail in the next section. New insight into the intermediate case of radiationless transitions has been provided by the experiments of Van der Meer et al. [1982b] on 
pyrazine. This work has been reviewed lucidly by Kommandeur et al. [1987]. For more recent work, see Tomer et al. [1988], Konings et al. [1988], Siebrand et al. [1989].

\subsection{Criteria for IVR regimes}

IVR phenomena are governed by the details of energy level density, $\rho_{l}$, by the decay rates $\left(\Gamma_{l}\right)$ due to infrared emission of the zero-order levels $\left\{\phi_{l}\right\}$, and the $s-l$ couplings between manifolds. Another important consideration is the nature of the initially prepared state. For instance, molecules are now commonly prepared with substantial amounts of energy in local modes [Swofford et al. 1976; Bray and Berry 1979], for recent reviews, see Crim [1984], Reisler and Wittig [1986]. Experiments are underway to study the subsequent time evolution, which will, one hopes, lead to unambiguous conclusions about the nature and rate of intramolecular processes from direct time-resolved measurements [Scherer et al. 1986; Scherer and Zewail 1987], rather than on the basis of optical linewidths [Rizzo et al. 1984; Ticich et al. 1986; Butler et al. 1986b; Luo et al. 1988].

On the other hand, if it were technically possible to excite an eigenstate of the molecular Hamiltonian, there should be very little (if any) nontrivial time evolution. To quantify the criteria that characterize the range of possible behavior, Freed [1981] defines the parameter

$$
X_{l}=\hbar \Gamma_{l} / \varepsilon_{l}
$$

where $\hbar \Gamma_{l}$ represents the energy width of a level with a decay rate of $\Gamma_{l}$, and $\varepsilon_{l}$ is the average spacing between the $\left\{\phi_{l}\right\}$ levels. The condition for the small-molecule limit is

$$
X_{1} \ll 1,
$$

whereas for the large molecule it is

$$
X_{l} \gg 1
$$

In the small-molecule limit, the situation corresponds to the spectroscopist's description of perturbations in the spectra of small molecules. The molecular Hamiltonian is represented in the basis set of zero-order vibrational eigenfunctions $\left\{\phi_{s}, \phi_{l}\right\}$. Coupling among only a few levels needs to be considered, namely, those which are nearly resonant and which have appreciable coupling matrix elements. These are levels for which

$$
\left|v_{s l}\right| /\left|E_{s}-E_{l}\right| \geq 1
$$

where $E_{s}$ and $E_{l}$ are the zero-order vibrational energies of $\phi_{s}$ and $\phi_{l}$, and $v_{s l}$ are the coupling matrix elements

$$
v_{s l}=\left\langle\phi_{s}|H| \phi_{l}\right\rangle
$$

This is the action of the Fermi resonance familiar to spectroscopists [Fermi 1931]. The partial, "local" diagonalization of the molecular Hamiltonian within the resonantly coupled states leads to the molecular eigenstates $\left\{\psi_{n}\right\}$, which are linear superpositions of the basis states, viz. 


$$
\psi_{n}=a_{s n} \phi_{s}+\sum_{j=1}^{N} a_{l_{j} n} \phi_{l_{j}},
$$

where $j$ counts the $\phi_{l}$ states ( $N$ in number) included in the superposition. Assuming that $\phi_{s}$ and $\phi_{l}$ do not radiate to any set of common levels, it is possible to evaluate the radiative decay rates of the molecular eigenstates $\left\{\psi_{n}\right\}$ as

$$
\Gamma_{n}=\left|a_{s n}\right|^{2} \Gamma_{s}+\sum_{j=1}^{N}\left|a_{l j n}\right|^{2} \Gamma_{l} .
$$

For many cases of interest the zeroth-order radiative decay rate of $\phi_{s}$ is much greater than that of the $\left\{\phi_{l}\right\}$. So, under the condition that

$$
\Gamma_{s} \gg \Gamma_{l}
$$

the radiative decay rates of the molecular eigenstates are equal to

$$
\Gamma_{n} \simeq\left|a_{s n}\right|^{2} \Gamma_{s}
$$

The condition that the original zeroth order states, $\phi_{s}$, be distributed amongst the molecular eigenstates implies the normalization condition

$$
\sum_{n=0}\left|a_{s n}\right|^{2}=1
$$

It follows from this that if more than one of the $a_{s n}$ are nonzero, we must have

$$
\left|a_{s n}\right|^{2}<1
$$

Equations (2.22) and (2.24) yield the result

$$
\Gamma_{n}<\Gamma_{s} \text { for } \Gamma_{s} \gg \Gamma_{l},
$$

which implies that the radiative decay rates of the mixed, molecular eigenstates $\left\{\psi_{n}\right\}$ are less than the radiative decay rate of the parent zeroth-order state $\phi_{s}$ carrying all of the original oscillator strength.

When the spacing between molecular eigenstates is large compared with the uncertainty widths of these levels, i.e.,

$$
\min \left|E_{n}-E_{n^{\prime}}\right| \gg \frac{1}{2} \hbar\left(\Gamma_{n}+\Gamma_{n^{\prime}}\right)
$$

monochromatic excitation can only lead to the preparation of individual molecular eigenstates. Pulsed excitation, on the other hand, can lead to the coherent excitation of a superposition of a number of nearly molecular eigenstates. However, when the pulse duration $\tau$ satisfies in addition to (2.26) the inequality

$$
\min \left|E_{n}-E_{n^{\prime}}\right|>\hbar / \tau,
$$


this pulsed excitation cannot excite more than one of the individual molecular eigenstates. If the pulse is sufficiently short or the spacing between molecular eigenstates is sufficiently small that the inequality (2.27) and/or (2.26) is violated, then a coherent superposition of these nearby molecular eigenstates is possible (for the subsequent dynamics, see, e.g., Taylor and Brumer [1983]).

The observation of irreversibility for the large-molecule case, eq. (2.16), has been explained by Freed [1981] from the radiationless transitions point of view. If such a molecule begins in state $\phi_{s}$ and crosses over to the $\left\{\phi_{l}\right\}$ manifold, these final states decay with rates $\Gamma_{l}$ before the molecule has a chance to cross back to the original state $\phi_{s}$. Thus it is the decay of the final manifold of levels $\left\{\phi_{1}\right\}$ that drives the irreversible relaxation from $\phi_{s}$ to $\left\{\phi_{l}\right\}$. There are many situations in which the inequality (2.21), generally true for condition (2.16), might seem inapplicable to such systems. However, in these cases one is primarily interested in whether or not the radiationless transition from $\phi_{s}$ to $\left\{\phi_{l}\right\}$ appears irreversible on the timescale of the experiment [Freed 1976]. When the decay properties of a system on a particular time scale, $\tau_{l}$, for an experiment are considered, the parameter (2.14) can be modified to read

$$
X_{l}^{\prime}=\hbar\left(\Gamma_{l}+\tau_{l}^{-1}\right) / \varepsilon_{l}
$$

and thus characterizes the decay characteristics of a molecule on the experimental timescale. When

$$
X_{l}^{\prime} \ll 1 \text {, }
$$

small-molecule behavior is manifest on the experimental timescale. Conversely if

$$
X_{l}^{\prime} \gg 1
$$

is obeyed, the decay characteristics of the molecule correspond to the statistical limit.

\subsection{Dephasing versus relaxation in isolated molecules}

It is always possible to view the time evolution of nonstationary states of a molecule as a dephasing process in the eigenstate representation, since these states are made up of a superposition of stationary states, each of which evolves differently in time - any phase relation established initially tends to be destroyed. On the other hand, this description of most processes as dephasing is neither particularly useful nor informative when we concentrate on what happens to a particular mode or a group of modes. In such a case, the terms "pure dephasing" (also called $T_{2}$ dephasing process) and "energy relaxation" ( $T_{1}$ process) are used to describe distinguishable appearances of the general dephasing process. In pure dephasing, the energy of a mode (or a collection of modes) does not change (e.g., Kay [1981], Stone et al. [1981], Budimir and Skinner [1987]). This process is caused by energy exchange among other modes in the molecule, which in turn causes the effective frequency of our chosen modes to fluctuate [Mukamel 1978; Makarov and Tyakht 1982; and Stuchebrukhov et al. 1989]. This terminology originated in solid state physics, where one is frequently interested in phenomena in a subsystem; and misleadingly and inaccurately, often dephasing is used to mean pure dephasing. On the other hand, in an energy relaxation process, the energy of our subsystem does change (see, e.g., Bloembergen and Zewail [1984], Stuchebrukhov et al. [1989]).

For concreteness, let us return to the "too-many-level small-molecule" case. As mentioned before, 
broadband excitation can produce an initially prepared nonstationary state of the molecule which closely approximates the state $\phi_{s}$ at $t=0$. The subsequent time evolution of the system is governed by the time evolution of the molecular eigenstates. The state of the system at time $t$ in this limit can be shown to be (in the notation of Freed [1981])

$$
\psi(t)=\sum_{n} a_{n s} \exp \left(-\mathrm{i} E_{n} t / \hbar-\frac{1}{2} \Gamma_{n} t\right) \psi_{n} .
$$

The probability of observing the decay characteristics of the state $\phi_{s}$ at time $t$ is proportional to the probability of finding the molecule in the zeroth-order state $\phi_{s}$ at $t$, which is given by (using the notation of Freed and Nitzan [1980])

$$
P_{s}(t) \equiv\left|\left\langle\phi_{s} \mid \psi(t)\right\rangle\right|^{2}=\left.\left.\left|\sum_{n}\right| a_{n s}\right|^{2} \exp \left(-\mathrm{i} E_{n} t / \hbar-\frac{1}{2} \Gamma_{n} t\right)\right|^{2} .
$$

Note that the probability is a complicated sum over molecular eigenstates with both oscillatory and damped time dependences. The general behavior of (2.32) could, in general, be very involved. However the large number of contributing terms provides a simplification. Again assume the validity of (2.21) and assume that $\Gamma_{l}$ is very small. However, on a short enough timescale, the modified condition (2.30) may be satisfied for time scales on the order of or less than the radiative decay rates, $\Gamma_{n}$, of the individual molecular eigenstates. Then, on the experimental timescale $\tau_{l}$ the molecule appears as if it conforms to the large-molecule statistical limit (2.16). Thus, as shown by Lahmani et al. [1974], $P_{s}(t)$ of (2.32) displays an exponential decay with a decay rate given by

$$
\Gamma_{s}^{\mathrm{tot}}\left(\tau_{l}\right)=\Gamma_{s}+\Delta_{s}
$$

where

$$
\Delta_{s}=\frac{2 \pi}{\hbar} \sum_{j}\left|v_{s l}\right|^{2} \rho_{l j}
$$

is the rate at which the molecule appears to undergo decay from $\phi_{s}$ to $\left\{\phi_{l}\right\}$ on the time scale $\tau_{l}$.

This short-time apparent decay is an intramolecular "dephasing" process, which is caused by the individual molecular eigenstates in (2.32) all having slightly different energies $\left\{E_{n}\right\}$ (recently, Makarov and Tyakht [1987] have studied purely phase relaxation and its effect on vibrational spectra. For a classical-mechanical "dephasing" study, see Farantos and Flytzanis [1987]). When the molecule is initially prepared in the nonstationary state $\phi_{s}$, a coherent superposition of all molecular eigenstates with fixed relative phases is constructed. However, because of the energy differences, these phases evolve at different time rates.

The intramolecular dephasing of the different molecular eigenstates is what leads to the apparent exponential decay for short times governed by (2.30), making it look as if the initial state $\phi_{s}$ is decaying into the $\left\{\phi_{l}\right\}$. To put it more picturesquely, under these conditions, this intermediate-case molecule has not had enough time to realize that there are only a finite number of levels $\left\{\phi_{l}\right\}$; it cannot resolve energy differences smaller than $1 / \tau_{\mathrm{e}}$. Therefore, because of time-energy uncertainty, it appears that $\left\{\phi_{l}\right\}$ is a continuum, and exponential decay ensues. This is an intramolecular dephasing process which, on this short timescale, is equivalent to IVR observed in the statistical limit (Carmeli et al. [1980], Gelbart et al. [1975]). At longer times, when (2.29) holds, the molecule has had time to find out that 
the manifold $\left\{\phi_{l}\right\}$ is discrete, and it reverts to small-molecule behavior (Heller [1981a,b] has reformulated the time-domain basis of spectroscopy in this fashion). Therefore, the initial exponential decay (known as "intramolecular dephasing") is not a truly irreversible relaxation because in principle, a multiple pulse sequence could be applied to the system to reconstitute the state $\phi_{s}$. The presence of a finite number of levels makes the dephasing reversible. In the statistical limit, the condition (2.16) implies the participation of an effectively infinite number of levels leading to irreversible relaxation.

On longer timescales where (2.30) is violated, the system returns to the small-molecule limit and the complicated expression (2.32) (for an experimental study of dephasing in an intermediate-case molecule, see Smith et al. [1983]). Ordinarily, the long-time behavior of such molecules is found to be adequately represented by a simple exponential decay with some average molecular eigenstate decay rate, $\left\langle\Gamma_{n}\right\rangle$. Between the short-time exponential decay of (2.33) and the long-time average molecular eigenstate exponential decay of $\left\langle\Gamma_{n}\right\rangle$, in principle, the decay given by (2.32) could be very complicated, including, e.g., quantum interference effects (see next section). The decay properties of these intermediate-case molecules in the too-many-level, small-molecule limit, are well represented by a biexponential decay involving the short-time apparent radiationless transition and the long-time decay of the individual molecular eigenstates. Muhlbach and Huber [1986] have reported an experimental observation of such biexponential decay recently. For an experimental discussion of biexponential decay, see Kommandeur et al. [1987].

\section{3. "Phenomenological" theories of energy sharing; approach of radiationless transitions}

There always has been a need for direct, dynamical IVR experiments [Mukamel and Smalley 1980; Moore et al. 1983; Imre et al. 1984; Sundberg and Heller 1984; Felker and Zewail 1984, 1985a-d; Dai et al. 1985a,b; Tannor et al. 1985; Heppener et al. 1985; Zewail 1985; Hamilton et al. 1986; Makarov 1987; Graner 1988] so that the sometimes puzzling outcomes of indirect experiments could be avoided. One class of experimental studies aims at detecting the time evolution of molecular fluorescence as a direct observation of IVR [Parmenter 1983; Coveleskie et al. 1985a,b; Dolson et al. 1985; Holtzclaw and Parmenter 1986; Knight 1988a,b]. These experiments observe the gradual filling-in of an initial sparse fluorescence spectrum because initially unexcited states are being populated by IVR. This time evolution has been observed in the "chemical timing" experiments of the Parmenter group, and provide an approximate picosecond timescale for IVR.

Briefly, a mode of a large molecule is excited and the fluorescence is detected. The experimental apparatus contains some quencher gas, which removes the excitation energy by means of collisional energy transfer. For very large pressures of quencher gas, the fluorescence spectrum is sparse because the excitation energy is removed before other modes can be populated by intramolecular energy flow. On the other hand, for very low gas pressures energy flow can proceed for a longer time, and the fluorescence spectrum looks much richer. Without quenching gas, a smooth spectrum is obtained, indicating that all coupled states are populated. One of the drawbacks of these procedures is that the range of observation times is limited to the fluorescence timescale.

The following theoretical description will specifically refer to relaxation in an electronically excited state, and how the progress of IVR affects the fluorescence spectrum We chose to restate the theory, which was explained by Freed and Nitzan [1980, 1983] for fluorescence, for two reasons: firstly, it is applicable to most classic manifestations of IVR; and secondly, while this theory was formulated, as has been traditional and useful, for fluorescence developing from IVR, it has some relevance for novel 
processes like vibrationally mediated photodissociation [Ticich et al. 1987; Sinha et al. 1987] which probe the interplay of IVR with photodissociation [Simons 1984].

The state densities in these molecules put them into an area where a theoretical framework analogous to the intermediate case in the theory of electronic relaxation processes should be applicable [Freed 1976, Tric 1976]. As stated before, in this intermediate case the density of final vibrational levels is insufficient to drive purely irreversible behavior on the timescale of the experiment (which is of the order of the fluorescence lifetime). In the literature of electronic relaxation, the intermediate case is clearly distinguished from the statistical case [Nitzan et al. 1972; Frad et al. 1974; Freed 1976; Mukamel and Jortner 1977]. But in the case of IVR there is a qualitative difference between these two scenarios: for instance, using the intermediate theory, it is possible to determine the average number of strongly coupled levels and the average coupling between the zeroth-order level and these strongly coupled levels. The difference between the intermediate theory, as formulated for electronic relaxation, and the version of the theory needed for IVR, is that in the electronically excited case, the dense manifold of levels which is strongly coupled to the initially excited one, is either nonemitting or is emitting on a timescale much longer than the experimental one. The current theory is characterized by a manifold of "final" levels whose emission is monitored by the experiment.

These generalizations have been clearly delineated in the work of Freed and Nitzan [1980], and can be used to distinguish between the intermediate and statistical behavior, and thus determine the threshold for irreversible vibrational relaxation. While most applications of this theory have been made to IVR taking place in excited electronic states, the experiments on relaxation in the ground electronic state (e.g., Minton and McDonald [1988]) indicate that there is very little, if any, difference between the scenarios (Parmenter [1983]), when there are no electronic curve-crossings.

The reverse procedure of deconvoluting spectra has recently been addressed by Lawrance and Knight [1988] using a Green function approach. This and similar work has been reviewed by Knight [1988a,b]. For a model Hamiltonian treatment in the spirit of this section, see Kommandeur et al. [1988].

\subsection{Molecular model for fluorescence}

The adiabatic Born-Oppenheimer approximation assumes that the wavefunctions of the system can be written in terms of a product of electronic, vibrational, rotational, and spin wavefunctions. If this approximation were exact, excitation and relaxation processes would be straightforward to describe. However, such a wavefunction is not an eigenfunction of the full molecular Hamiltonian, and numerous interactions, which are ignored by the Born-Oppenheimer approximation, can couple these states, leading to the removal of degeneracies and the occurrence of radiationless, e.g., transition processes. In what follows, we have adopted the reasoning and notation of Freed and Nitzan [1980]. For a recent exposition, with detailed references to current experiments, see the reviews by Knight [1988a,b].

The ideal experimental scenario finds the molecule initially in the ground vibrational-rotational level of the ground electronic state. Such ideal conditions can be approximated in supersonic jet experiments. For definiteness, suppose that the excitation is taking place into the molecular vibronic levels of the first excited molecular singlet, and ignore electronic radiationless relaxation. Conventional spectroscopical practice assigns the spectrum in terms of a basis set of zero-order levels based on the populations of a set of zero-order modes (which may be normal modes or symmetrized local modes, to cite two common examples).

The zero-order states may be divided into two groups, which are denoted by a for active, and by $b$ for bath. The a modes are those whose potential energy surfaces in the ground $(\mathrm{g})$ and electronic 
excited (e) states may differ appreciably from each other. The b modes do not change during this electronic transition. The a modes are optically active, "bright" states and thus correspond to the different progressions in the molecular absorption spectrum. The zero-order molecular vibronic levels are denoted $\left|\mathrm{g}, n_{\mathrm{ga}}, n_{\mathrm{gb}}\right\rangle$, and $\left|\mathrm{e}, n_{\mathrm{ea}}, n_{\mathrm{eb}}\right\rangle$, where $n_{i \mathrm{a}, i \mathrm{~b}}, i=\mathrm{g}$, e denote the populations of the a and $\mathrm{b}$ modes in the $\mathrm{g}$ and $\mathrm{e}$ electronic states. It should be noted that these $n$ stand for the collection of all modes $\mathrm{a}$ and $\mathrm{b}$ in this system. In this notation, the initial state can be abbreviated as $|\mathrm{g}, 0,0\rangle$.

In monitoring molecular fluorescence, one in fact monitors the populations of the a modes. The proper molecular model (fig. 2) is composed of manifolds of b states seated on the different states of the modes. All these states are the eigenstates of the zero-order molecular Hamiltonian $H_{0}^{\mathrm{M}}$. The total Hamiltonian governing the time evolution is

$$
H=H_{0}+\mu+W, \quad H_{0}=H_{0}^{\mathrm{M}}+H_{0}^{\mathrm{R}},
$$

where $H_{0}^{\mathrm{R}}$ is the Hamiltonian of the free radiation field, $\mu$ is the molecule-radiation field interaction which couples the e and $g$ states and $W$ is the intramolecular vibrational interaction which couples the different $a$ and $b$ states. IVR then is viewed as transitions between the manifolds shown in fig. 2 , induced by the off-diagonal coupling $W$. Sometimes it may be necessary to have two varieties of $W$, one that couples a and b modes, and the other the b modes among themselves. Obviously, the dynamics is especially sensitive to the first variety of coupling.

\section{2. "Exact" molecular eigenstates}

It is convenient (though not widely customary) to analyze the basics of the IVR process not in terms of "zero-order" states, i.e., the eigenstates of $H_{0}$, but in terms of the exact eigenstates, i.e., the

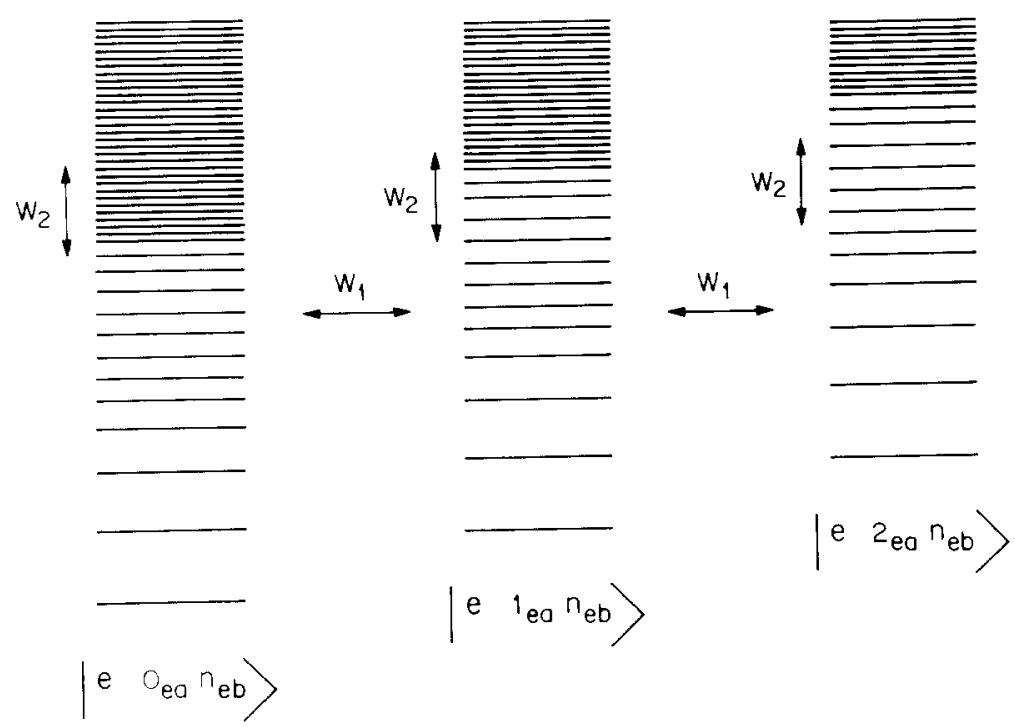

Fig. 2. The molecular model used in the discussion of intramolecular vibrational relaxation (IVR) in an excited electronic state (|e $\rangle$ ). Each manifold corresponds to a particular state of the optically active (a) modes and is composed of levels associated with different states of all other (bath, b) modes. $W_{1}$ denotes an intramolecular coupling between the a modes and the $\mathrm{b}$ modes (which leads to processes which change the populations of the a modes). $W_{2}$ denotes the coupling between the b modes. (From Freed and Nitzan [1980]). 
eigenstates of $H_{0}^{\mathrm{M}}+W+H_{0}^{\mathrm{R}}$. These states can be written as combinations of the zero-order states as

$$
\left|\psi_{\mathrm{e} j}\right\rangle=\sum_{n_{\mathrm{a}}, n_{\mathrm{b}}} C_{n_{\mathrm{a}}, n_{\mathrm{b}}}\left|\mathrm{e}, n_{\mathrm{a}}, n_{\mathrm{b}}\right\rangle \equiv|\mathrm{e}, j\rangle
$$

(here, we have suppressed the states of the radiation field $H_{0}^{\mathrm{R}}$ ). A narrow pulse which induces a transition from the ground state $|\mathrm{g}, 0,0\rangle$ to another particular zero-order level $\left|\mathrm{e}, n_{\mathrm{a}}, 0\right\rangle$ encompasses, in the exact molecular eigenstates picture, those molecular eigenstates $|\mathrm{e}, j\rangle$ which contain contributions from $\left|\mathrm{e}, n_{\mathrm{a}}, 0\right\rangle$,

$$
|\mathrm{e}, j\rangle=A_{j ; n_{\mathrm{a}}, 0}\left|\mathrm{e}, n_{\mathrm{a}}, 0\right\rangle+\sum_{n_{\mathrm{a}^{\prime}, n_{\mathrm{b}}^{\prime}}} B_{j, n_{\mathrm{a}}^{\prime}, n_{\mathrm{b}}^{\prime}}\left|\mathrm{e}, n_{\mathrm{a}}^{\prime}, n_{\mathrm{b}}^{\prime}\right\rangle \text {. }
$$

Assuming that the intramolecular coupling $W$ is much smaller than the energy spacing between the primary zero-order states $\left|\mathrm{e}, n_{\mathrm{a}}, 0\right\rangle$ leads to the following picture. Each exact $|\mathrm{e}, j\rangle$ state corresponds to a single primary $\left|\mathrm{e}, n_{\mathrm{a}}, 0\right\rangle$ state and a group of quasidegenerate $\left|\mathrm{e}, n_{\mathrm{a}}^{\prime}, n_{\mathrm{b}}^{\prime}\right\rangle$ states with $n_{\mathrm{a}}^{\prime}<n_{\mathrm{a}}$. The number of zero-order states contributing to the right-hand side of eq. (3.3) is of the order of $(|W| \rho)^{2}$, where $\rho$ is the density of levels in the $\left\{\left|\mathrm{e}, n_{\mathrm{a}}^{\prime}, n_{\mathrm{b}}^{\prime}\right\rangle\right\}$ manifold.

In the Condon approximation, the total radiative lifetimes of the different rovibronic states $\left|\mathrm{e}, n_{\mathrm{a}}, n_{\mathrm{b}}\right\rangle$ are the same for all levels. Therefore, the same is also true for the levels $|\mathrm{e}, j\rangle$ which diagonalize the molecular Hamiltonian. The total width of a zero-order molecular level $|\mathrm{e}, n\rangle$, where $n=\left(n_{\mathrm{a}}, n_{\mathrm{b}}\right)$, is

$$
\gamma_{n}=\gamma^{\mathrm{R}}+\gamma_{n}^{\mathrm{NR}}
$$

where $\gamma^{\mathrm{R}}$ is the radiative width and $\gamma_{n}^{\mathrm{NR}}$ is the nonradiative width associated with radiationless electronic transitions.

Let us begin by discussing the IVR problem using a much simplified scenario. If the molecule is initially cold, i.e., in the state $|\mathrm{g}, 0,0\rangle$, an incident pulse which is extremely narrow in energy may induce transitions to a single $\left|\mathrm{e}, n_{\mathrm{a}}, 0\right\rangle$ level. This assumption is valid if the spacing between $\left|\mathrm{e}, n_{\mathrm{a}}, 0\right\rangle$ levels is larger than the bandwidth of the radiation. Under these conditions, the excited zero-order states which participate in the absorption-emission process are the state $\left|\mathrm{e}, n_{\mathrm{a}}, 0\right\rangle$ and a group of states $\left|\mathrm{e}, n_{\mathrm{a}}^{\prime}, n_{\mathrm{b}}^{\prime}\right\rangle$ which are degenerate with it to within an energy range of order $W$. The latter are radiatively coupled to the ground levels (other than $\left|\mathrm{g}, n_{\mathrm{a}}^{\prime \prime}, 0\right\rangle$, which is coupled to $\left|\mathrm{e}, n_{\mathrm{a}}, 0\right\rangle$ ), and relaxed fluorescence arises from this coupling. Denote

$$
\left|\mathrm{e}, n_{\mathrm{a}}, 0\right\rangle \equiv|s\rangle, \quad\left\{\left|\mathrm{e}, n_{\mathrm{a}}^{\prime}, n_{\mathrm{b}}^{\prime}\right\rangle\right\} \equiv\{|\mathrm{e}\rangle\}
$$

Ground-state levels to which $|s\rangle$ and $|l\rangle$ are coupled radiatively are denoted by $\{|m\rangle\}$. We sometimes use $\left|m_{s}\right\rangle$ to denote a level (like $|\mathrm{g}, 0,0\rangle$ ) which is coupled to $|s\rangle$ but not to the levels $\{|l\rangle\}$. The model is shown in fig. 3a. In the corresponding exact molecular states picture, fig. 3b, the group of levels $|s\rangle$, $\{|l\rangle\}$ is replaced by the group $\{|j\rangle\}$ which are obtained as a linear combination of $|s\rangle$ and $\{|l\rangle\}$ states which diagonalize the hamiltonian $H_{0}^{\mathrm{M}}+W$.

The most significant difference between the model discussed here and similar models used before for the theory of electronic radiationless transitions is that the underlying continua (fig. 3) which provide the "bath" for the decay of the initially prepared level, are optically active. The emission from these 
(0)

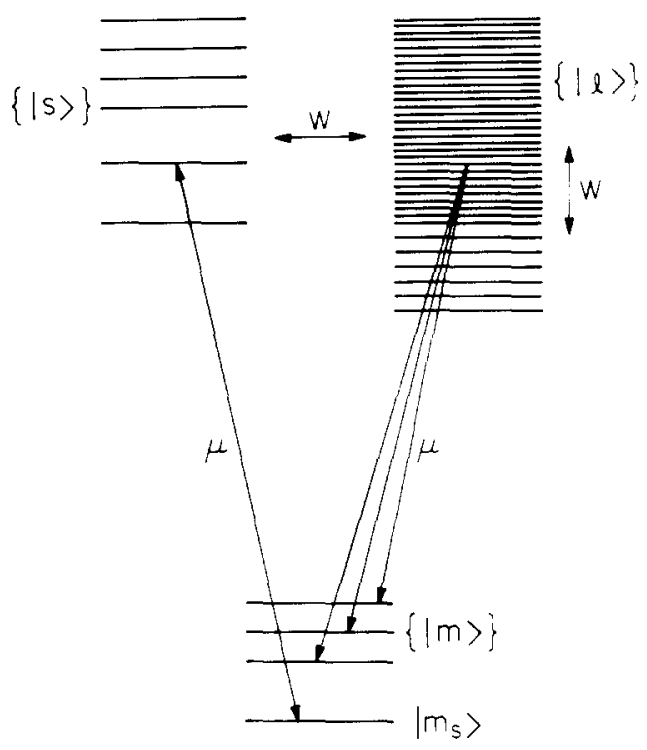

b!

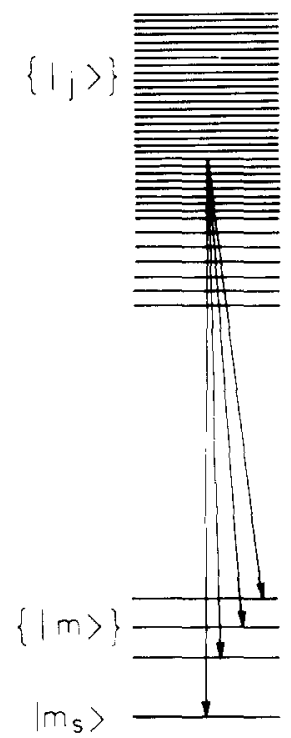

Fig. 3. A simplified molecular model. a. $|s\rangle$ are zero-order levels belonging to the excited electronic state which are directly accessible from the initial ground state level $\left|m_{s}\right\rangle$. The intramolecular zero-order bath levels are $\{|l\rangle\}$. b. The corresponding exact molecular state picture. (From Freed and Nitzan [1980]).

states constitutes the relaxed part of the fluorescence as discussed above. Now we explore the implications of this emission process on the line shape and time evolution of molecular fluorescence spectra.

\subsection{Long-time experiments}

In the intermediate level structure case, the decay widths $\gamma_{j}$ (eq. 3.4) of the individual exact molecular levels are smaller than their averaged spacing

$$
\gamma_{j}<(\hbar \rho)^{-1},
$$

with $\rho$ being the density of states. This implies that in a continuous excitation experiment or using a pulse long enough to resolve the individual levels the resulting fluorescence arises from an individual level $|\mathrm{e}, j\rangle$ (eq. 3.3). Therefore:

(1) In a long-time pulse experiment the fluorescence decays exponentially with lifetime $\tau_{j}=\hbar / \gamma_{j}$.

(2) The relative intensities of different absorption or fluorescence excitation lines (corresponding to different $|\mathrm{e}, j\rangle$ levels) are given by [cf. eq. (3.4)] $\left|A_{j ; n_{\mathrm{a}}, 0}\right|^{2}$.

(3) The fluorescence spectrum does not evolve in time.

In practical experimental scenarios, these conclusions (which apply to an ideal case) apply with modifications. For one thing, even in the coldest supersonic beam experiments, the initial state is not a single molecular eigenstate, but includes at least a few rotational states. Hence, the fluorescence spectrum contains contributions from a number of initial rotational levels, and this superposition tends to wash out some of the described structure. Note that IVR does not lead to broadening of individually 
resolved molecular transitions in the intermediate case, whereas there is broadening in the extreme statistical limit. As the density of states increases, the energy spacing becomes smaller than the widths $\hbar \gamma_{j}$ and so it becomes impossible to excite individual molecular eigenstates even with an ideally monochromatic radiation.

In the statistical limit (see below), the manifolds $\left|\mathrm{e}, n_{\mathrm{a}}^{\prime}, n_{\mathrm{b}}^{\prime}\right\rangle$ become dissipative continua for intramolecular decay of the initially excited level $\left|\mathrm{e}, n_{\mathrm{a}}, 0\right\rangle$. The decay rate is then given by the Golden Rule formula

$$
\Gamma_{n}=2 \pi \sum_{n_{\mathrm{a}}^{\prime}<n_{\mathrm{a}}}\left[\sum_{n_{\mathrm{b}}^{\prime}}\left|\left\langle\mathrm{e}, n|W| \mathrm{e}, n^{\prime}\right\rangle\right|^{2} \delta\left(\sum_{\alpha} \omega_{\mathrm{e} \alpha} n_{\mathrm{a}}^{\alpha^{\prime}}-\sum_{\beta} \omega_{\mathrm{e} \beta} n_{\mathrm{b}}^{\beta^{\prime}}\right)\right],
$$

where $n$ and $n^{\prime}$ are short-hand for $\left(n_{\mathrm{a}}, 0\right)$ and $\left(n_{\mathrm{a}}^{\prime}, n_{\mathrm{b}}^{\prime}\right)$. The absorption lineshape to the resonance centred around the zero-order state $|\mathrm{e}, n, 0\rangle$ becomes, in the extreme statistical limit, approximately a Lorentzian with a width $\Gamma_{n}+\gamma_{n}$ consisting of contributions corresponding to vibrational, radiationless electronic, and radiative relaxation. The relative yields of the direct versus relaxed fluorescence can be calculated by using a "kinetic" approach [Nitzan et al. 1971; Mukamel and Nitzan 1977], which is justified in this case because the random nature of the coupling matrix elements ensures the absence of interference effects [Freed and Nitzan 1980].

\subsection{Short-time experiments}

Now we consider the case where the excitation pulse is short enough in time, and broad enough in energy so that a zero-order nonstationary state $\left|\mathrm{e}, n_{\mathrm{a}}, 0\right\rangle$ is prepared. In terms of exact molecular eigenstates $|\mathrm{e}, j\rangle$ the initial state is [cf. eq. (3.3)]

$$
|\psi(0)\rangle=\left|\mathrm{e}, n_{\mathrm{a}}, 0\right\rangle=\sum_{j} A_{j ; n_{\mathrm{a}}, 0}^{*}|\mathrm{e}, j\rangle .
$$

To achieve this initial excitation, the pulse time has to be much shorter than the inverse energy span of the states $|\mathrm{e}, j\rangle$ with $A_{j: n_{\mathrm{a}}, 0}$ different from zero (fig. 4). The pulse bandwidth has to be smaller than the energy spacing between $\left|\mathrm{e}, n_{\mathrm{a}}, 0\right\rangle$ levels. Assuming that these conditions hold, we can analyze the molecular fluorescence in terms of the simplified model defined in section 3.2 and depicted in fig. 3. In terms of this model, the zero-order level structure, the corresponding exact-states manifold and a characteristic absorption spectrum corresponding to the sparse intermediate (of level spacings larger than level widths) and the dense intermediate (level spacings much smaller than level widths) cases are shown in fig. 5 . Note that by level widths we mean the sum of radiative and electronic nonradiative widths rather than widths associated with IVR.

To appreciate the significance of the emission spectra displayed in fig. 5, consider first the two emission lines which correspond to two different final (ground state) levels $\left|m_{s}\right\rangle$ and $\left|m_{r}\right\rangle$. Suppose that in the zero-order representation, $\left|m_{s}\right\rangle$ is exclusively coupled (via $\mu$, the molecule-radiation field interaction) to $|s\rangle$, while $\left|m_{r}\right\rangle$ is coupled to another zero-order level $|r\rangle$ which belongs to the $\{|l\rangle\}$ manifold, i.e., if the molecule is initially in $\left|m_{s}\right\rangle$, a broad-band pulse will prepare it in state $|s\rangle$. If the initial state is $\left|m_{r}\right\rangle$, similar excitation will yield the state $|r\rangle$. Figure 6 shows this situation in the dense intermediate case together with the schematic description of the emission lineshape for transitions that end in the $\left|m_{s}\right\rangle$ and $\left|m_{r}\right\rangle$ levels. Note that by definition, the $|s\rangle \rightarrow\left|m_{s}\right\rangle$ transition constitutes a line in the direct (unrelaxed) fluorescence spectrum, whereas the $|r\rangle \rightarrow\left|m_{s}\right\rangle$ emission constitutes one (of many 


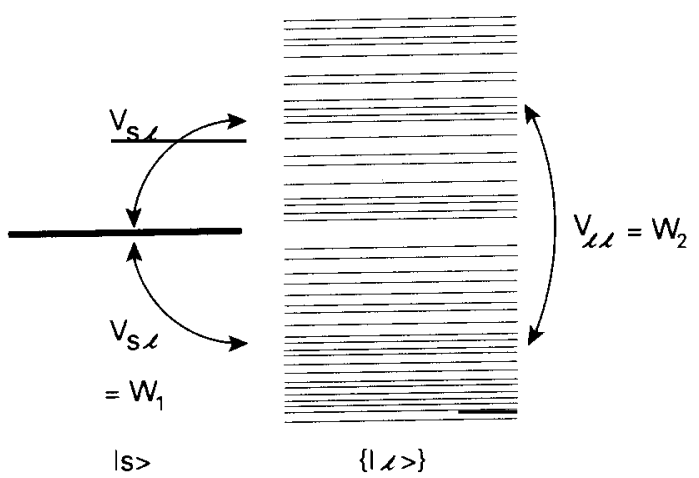

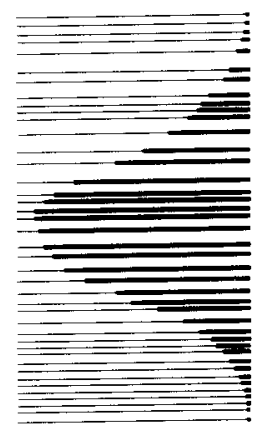

$\{\mid j>\}$

Fig. 4. Schematic diagram of the mixing of zero-order vibrational levels (or rovibrational levels) within a single electronic state. The average coupling matrix elements $V_{s l}$ and $V_{l l}$ are expected to be equal. Within the $\mathrm{S}_{1}$ state, the state $|s\rangle$ has a large Franck-Condon factor so that it is optically accessible from the $\mathrm{S}_{0}$ zero-point level. The bath levels $\{|l\rangle\}$ are "dark" on account of small Franck-Condon factors. The $|j\rangle$ are the exact molecular eigenstates $|\mathrm{e}, j\rangle$; the figure on the right shows the initial excited state in terms of a superposition of molecular eigenstates. This is a pictorial representation of the state that evolves according to (2.32) or (3.13). (Adapted from Parmenter [1983]).

overlapping) contributions to the relaxed emission (see also Mukamel [1985]). $|s\rangle$ and $|r\rangle$ are, however, linear combinations of the exact energy eigenstates $|j\rangle$. Therefore, in the intermediate regime, each of these lines could, in principle, be resolved to the different $|j\rangle$ levels, as shown in fig. 6.

At any time following the initial preparation of the excited molecule, the integrated intensity of the emission associated with the transition into the ground level $\left|m_{p}\right\rangle$ is proportional to the population in level $|p\rangle$, i.e. $|\langle p \mid \psi(t)\rangle|^{2}$, where $|\psi(t)\rangle$ is the molecular state at time $t$, and $|p\rangle$ is the excited zero-order state which carries all the oscillator strength for transitions from $\left|m_{p}\right\rangle$ in the spectral region of interest [Mukamel and Jortner 1977]. Note that $|p\rangle$ can be the initially excited level $|s\rangle$ or any other
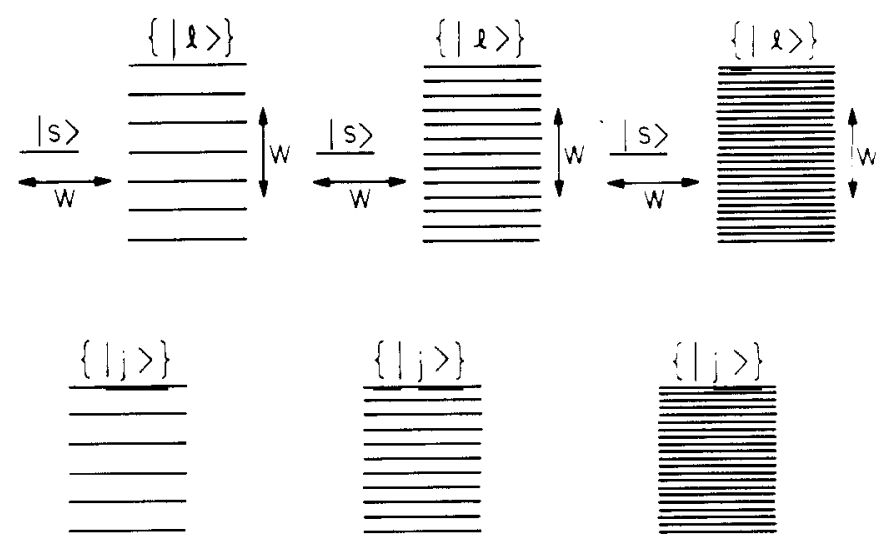

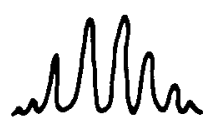

SPARSE

INTERMEDIATE

CASE

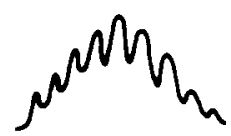

DENSE INTERMEDIATE CASE

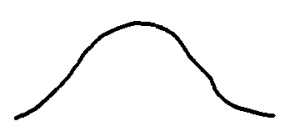

STATISTIEAL

LINIT

Fig. 5. Zero-order level structure (upper), exact molecular level structure (middle), and the corresponding absorption spectrum associated with the sparse intermediate case (left), the dense intermediate case (middle), and the statistical limit (right). (From Freed and Nitzan [1980]). 

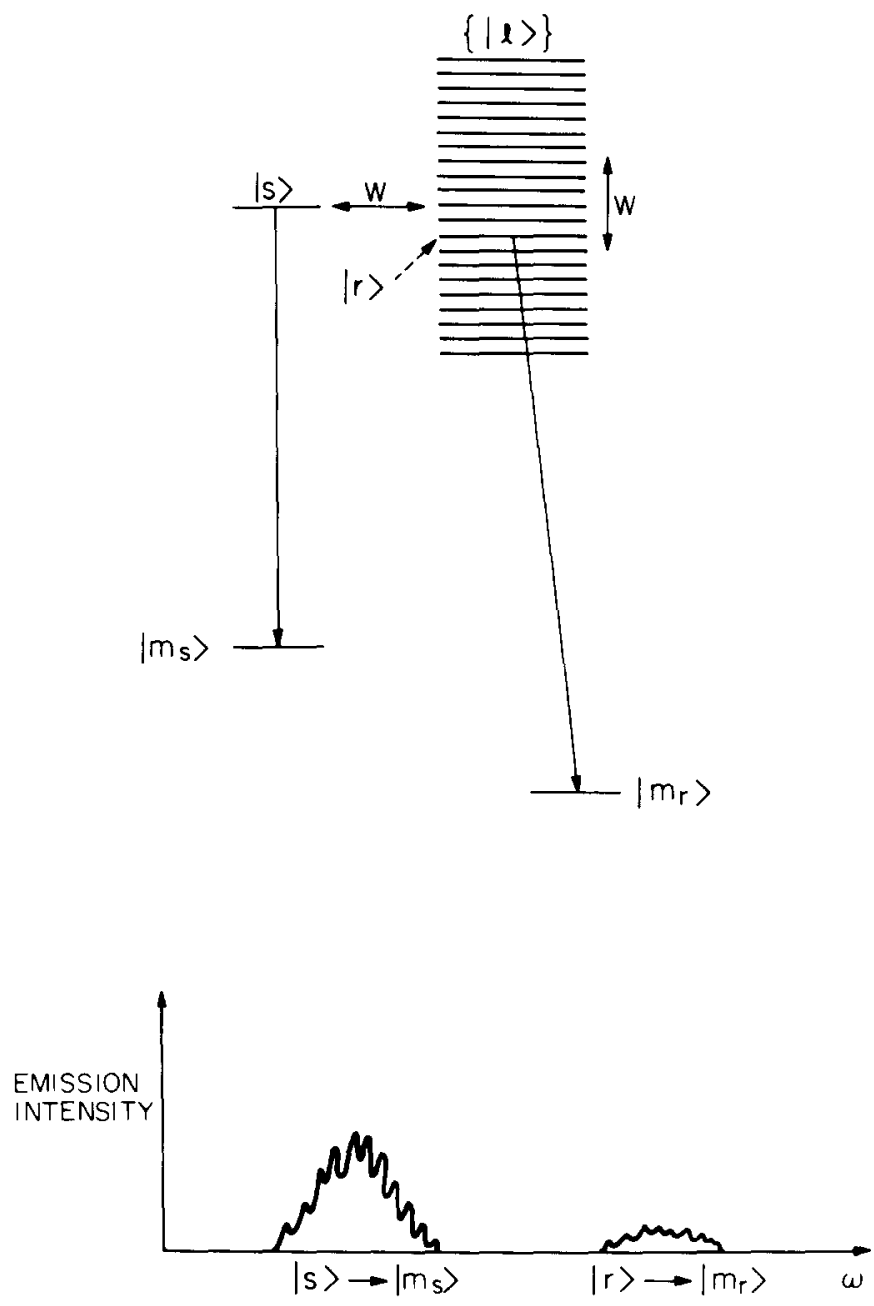

Fig. 6. Direct (unrelaxed, $|s\rangle \rightarrow\left|m_{s}\right\rangle$ ) and indirect (relaxed, $|r\rangle \rightarrow\left|m_{r}\right\rangle$ ) emissions in the simplified molecular model. Note that the $|r\rangle \rightarrow\left|m_{r}\right\rangle$ emission is only one component contributing to the total relaxed fluorescence. (From Freed and Nitzan [1980]).

zero-order level belonging to the manifold $\{|l\rangle\}$. In the rest of this section we assume that the condition under which the populations of the zero-order levels determine the instantaneous emission spectra is satisfied. The intensity of the direct (unrelaxed) emission at time $t$ is proportional to the population

$$
P_{s}(t)=|\langle s|\exp (-\mathrm{i} H t)| s\rangle|^{2}
$$

(henceforth we set $\hbar=1$ ), and the spectrally integrated intensity of the relaxed emission at a time $t$ is proportional to

$$
P_{L}(t) \equiv \sum_{l} P_{l}(t)=\sum_{l}|\langle l|\exp (-\mathrm{i} H t)| s\rangle|^{2}
$$

The evolution within the $|s\rangle-\{|l\rangle\}$ manifold in the model described in fig. 6 has been intensively studied in connection with the theory of intramolecular electronic relaxation. In the intermediate case 
the population $P_{s}(t)$ evolves differently at short and long times. Immediately following the excitation, i.e., after $|\psi(t=0)\rangle=|s\rangle$ and during a time short compared to the inverse level spacing in the $\{|l\rangle\}$ manifold, the $\{|l\rangle\}$ manifold behaves as a continuum and the evolution of $P_{s}(t)$ proceeds as in the statistical limit (see below). The direct emission decays with the total rate $\gamma_{s}+\Gamma$, where $\gamma_{s}$ is the sum of the radiative and electronic radiationless widths of the levels $|s\rangle$ while $\Gamma$ is given by

$$
\Gamma=2 \pi \sum\left|W_{s l}\right|^{2} \frac{1}{\pi} \frac{\frac{1}{2}\left(\gamma_{l}+\varepsilon_{0}\right)}{\left(E_{s}-E_{l}\right)^{2}+\frac{1}{4}\left(\gamma_{l}+\varepsilon_{0}\right)^{2}} \simeq 2 \pi\left\langle\left|W_{s l}\right|^{2} \rho_{l}\right\rangle
$$

where $\gamma_{l}$ is the sum of the radiative and electronic nonradiative widths of the level $|l\rangle, \rho_{l}$ is the density of levels in the $\{|l\rangle\}$ manifold and $\varepsilon_{0}$ is the uncertainty width (of the order $\tau_{0}^{-1}$ ) associated with the short observation time $\tau_{0}$ [Freed 1970].

At long times ( $t$ greater than $\hbar \rho_{l}$ ), dephasing between the different levels due to their slightly different energies has taken place (however, in principle, this time may also be long enough for recurrences to take place). As in the previous section, the situation is best described in terms of the exact molecular eigenstates $|j\rangle$. The initial state is

$$
|\psi(t=0)\rangle=|s\rangle=\sum_{j} a_{s j}|j\rangle
$$

and at a time $t$ it evolves to

$$
|\psi(t)\rangle=\sum_{j} a_{s j} \exp \left(-\mathrm{i} E_{j} t-\gamma_{j} t\right)|j\rangle
$$

Equation (3.13) is based on the assumption that the damping matrix associated with the exact molecular eigenstates $|j\rangle$ is diagonal. This may be justified for the radiationless part of $\gamma_{j}$ invoking the random-like nature of intramolecular coupling between vibrational levels. It is also true for the radiative part of $\gamma_{j}$ because the total radiative lifetime is the same for all rovibronic levels corresponding to a given electronic state. Equation (3.13) leads to

$$
P_{s}(t)=|\langle s \mid \psi(t)\rangle|^{2}=\sum_{j}\left|a_{s j}\right|^{4} \exp \left(-\gamma_{j} t\right)+2 \sum_{j \neq j^{\prime}}\left|a_{s j}\right|^{2}\left|a_{s j^{\prime}}\right|^{2} \exp \left[-\frac{1}{2}\left(\gamma_{j}+\gamma_{j^{\prime}}\right) t\right] \cos \left(\omega_{j j^{\prime}} t\right)
$$

where $\hbar \omega_{i j^{\prime}}=E_{j}-E_{j}$. The second term in the right-hand side of eq. (3.14) describes quantum beats in the fluorescence. However, if more than just a few levels contribute, the sum of oscillating terms averages for $t>\hbar \rho_{j}$ to a vanishingly small contribution. This subject is further discussed in the next section. When these oscillations may be disregarded, we obtain (for long enough time)

$$
P_{s}(t)=\sum_{j}\left|a_{s j}\right|^{4} \exp \left(-\gamma_{j} t\right)
$$

If there are approximately $N>2$ strongly coupled $s$ and $l$ levels participating in this process, we may invoke the simplest statistical model of egalitarian mixing so that

$$
\left|a_{s j}\right|^{2}=N^{-1}
$$


We then have

$$
P_{s}(t)= \begin{cases}\exp \left[-\left(\gamma_{s}+\Gamma\right) t\right] & \text { (short time) } \\ (1 / N) \exp \left(-\gamma_{j} t\right) & \text { (long time) }\end{cases}
$$

The number $N$ of effectively coupled levels may be estimated from

$$
N \cong \Gamma \rho=2 \pi|W|^{2} \rho^{2}
$$

The only difference between the present situation and that encountered in the theory of electronic relaxation lies in the fact that in most electronic relaxation problems the real-life counterpart of the manifold $\{|l\rangle\}$ does not carry significant oscillator strength for radiative emission. The observed radiative lifetime (that is, the radiative part in $\gamma_{j}$ ) is then of the order $\gamma_{s}^{\mathrm{R}} / N$. In the current treatment, $\gamma_{s}^{\mathrm{R}}, \gamma_{l}^{\mathrm{R}}$ and $\gamma_{j}^{\mathrm{R}}$ are all equal.

The level widths

$$
\gamma_{s}=\gamma_{s}^{\mathrm{R}}+\gamma_{s}^{\mathrm{NR}}
$$

and

$$
\gamma_{l}=\gamma_{l}^{\mathrm{R}}+\gamma_{l}^{\mathrm{NR}}
$$

have approximately the same order of magnitude. If we make the simplifying assumption that they are the same (which is rigorously true if variations in the nonradiative width are neglected), we can obtain also the time evolution of the integrated relaxed fluorescence. The individual $P_{l}(t)$ are fluctuations within the egalitarian model, having zero average. Their sum, however, is readily evaluated by using the sum rule

$$
P_{s}(t)+\sum_{l} P_{l}(t)=\exp (-\gamma t)
$$

where $\gamma=\gamma_{s}=\gamma_{l}$, to obtain

$$
\begin{array}{ll}
\sum_{l} P_{l}(t)=\exp (-\gamma t)[1-\exp (-\Gamma t)] & \text { (short time) } \\
\sum_{l} P_{l}(t)=\frac{N-1}{N} \exp (-\gamma t) & \text { (long time). }
\end{array}
$$

Equations (3.17), (3.18) and (3.23) lead to the following important conclusion. When quantum beats in the fluorescence are absent, the time evolution of the molecular fluorescence spectrum (i.e., transfer of intensity from direct to relaxed emission bands) takes place only during the initial short dephasing period. This may also be concluded using the exact molecular states representation. Following the initial dephasing, each level $|j\rangle$ evolves in time essentially independently of other levels and contributes to the emission spectrum direct and relaxed components as discussed in section 3.3. These components decay with lifetime $\gamma_{j}$ and any evolution in the structure of the emission spectrum in the post-dephasing period is due to the accidental differences between different lifetimes $\gamma_{j}^{-1}$ of the different $j$ levels. 


\subsubsection{The statistical limit}

As seen from eqs. (3.17) and (3.18), as the number $N$ of effectively coupled levels within the $|s\rangle-\{|l\rangle\}$ manifold becomes very large, the long-time component in the evolution of $P_{s}(t)$ becomes negligibly small, $P_{s}(t)$ decays exponentially practically to zero with the rate $\gamma_{s}+\Gamma$. In most cases of electronic radiationless relaxation, fluorescence is observed to decay on this timescale. In the present case eq. (3.23) indicates that the total population of the $\{|l\rangle\}$ states, which determine the intensity of the relaxed fluorescence, decay on the long-time scale with a rate $\gamma=\langle\gamma\rangle$ following an initial rapid rise. The situation is very similar to that discussed by Nitzan et al. [1972] in connection with consecutive electronic relaxation. It is important to note that as long as quantum beats are not observed and as long as no attempt is made to resolve the intermediate level structure in the emission, the statistical limit and the intermediate case differ only by the magnitude of the number $N$ which enters into eqs. (3.17), (3.18) and (3.23).

An interesting question that has not been addressed before concerns the population of different levels $|l\rangle$ in the $\{|l\rangle\}$ manifold during the vibrational relaxation. Consider again the model shown in fig. 6 , and focus attention on the statistical limit (or the short-time evolution in intermediate cases). Usually, the time evolution within the $|s\rangle-\{|l\rangle\}$ manifold is determined by disregarding the anharmonic coupling $W$ between the $|l\rangle$ levels, denoted by $W_{2}$ in fig. 2 . The usual argument given is that as long as we are interested only in $P_{s}(t)$ we can perform a partial diagonalization of the Hamiltonian matrix and obtain a set of $\{|l\rangle\}$ states which are not coupled to each other. The results for the populations $P_{s}(t)$ and $P_{l}(t)$ for this case are well-known [Goldberger and Watson 1969],

$$
\begin{aligned}
& P_{s}(t)=\exp [-(\gamma+\Gamma) t] \\
& P_{l}(t)=\frac{\left|W_{l s}\right|^{2} \exp (-\gamma t)}{E_{l s}^{2}+\left(\frac{1}{2} \Gamma\right)^{2}}\left[1+\exp (-\Gamma t)-2 \exp \left(-\frac{1}{2} \Gamma t\right) \cos \left(E_{l s} t\right)\right], \\
& E_{l s}=E_{l}-E_{s}, \quad \Gamma=2 \pi\left\langle W_{s l}^{2} \rho_{l}\right\rangle .
\end{aligned}
$$

In the present case we are interested not only in $P_{s}(t)$, but also in $P_{l}(t)$. The sum $\Sigma_{l} P_{l}(t)$ which determines the integrated relaxed fluorescence is given by eq. (3.23). Individual populations $P_{l}(t)$ may be useful for sorting out different contributions to the relaxed spectrum. For this purpose we would like to keep the original character and symmetry of the $|l\rangle$ states, and in particular the selection rules associated with their symmetry. We therefore need a solution to the problem where the coupling between the $|l\rangle$ states is not disregarded.

Such a solution can be obtained in the statistical limit within the framework of the random coupling model [Kay 1974; Heller and Rice 1974; Gelbart et al. 1975; Druger 1977a,b; Carmeli and Nitzan 1980a,b; Carmeli et al. 1980]. In this model, the coupling matrix elements between any two states in the $|s\rangle-\{|l\rangle\}$ manifolds are regarded as random functions of the state index. This picture is justified for matrix elements involving excited vibrational wavefunctions because of the highly oscillatory nature of these functions. We are interested in $P_{s}(t)$ and $P_{r}(t)$, given that $P_{s}(t=0)=1$, where the levels $|s\rangle$ and $|r\rangle$ are particular members of a dense set of coupled states. The level $|s\rangle$ is unique only because it is the only one accessible from the ground state and may therefore be prepared initially. The results are [Freed and Nitzan 1980]

$$
P_{s}(t)=\exp [-(\gamma+\Gamma) t]
$$




$$
P_{r}(t)=\frac{2\left\langle W^{2}\right\rangle \exp (-\gamma t)}{E_{r s}^{2}+\Gamma^{2}}\left\{1-\exp (-\Gamma t) \cos \left(E_{r s} t\right)-\left(\Gamma / E_{r s}\right) \exp (-\Gamma t) \sin \left(E_{r s} t\right)\right\}
$$

As expected, the time evolution of the initially populated state is the same in this model as for the simplified model which leads to eq. (3.24). The time dependence of $P_{r}(t)$ (for $r$ different from $s$ ) is different, but the qualitative behavior is similar. The most significant difference between eqs. (3.24b) and (3.26) lies in the fact that the latter predicts that the population spread over zero-order levels in a (zero-order) energy range is twice as large as that obtained in eq. (3.24b). This occurs because in the model which leads to (3.26), each level has an anharmonic width $\Gamma$, while in the model without coupling between the $\{|l\rangle\}$ levels only the initially excited level is assigned such a width.

\subsection{Quantum beats}

In bulk room temperature experiments involving relatively large numbers of molecules, quantum beats in the fluorescence are not observed. The initial thermal distribution of rotational states is carried over into the excited state and any oscillations in the fluorescence originating in these incoherently excited rotational levels are averaged out. With low beam temperatures the rotational structure can be eliminated and beats are observed (e.g., Laubereau et al. [1976], Chaiken et al. [1979], Van der Meer et al. [1982a], Felker et al. [1982], Okajima et al. [1982], Felker and Zewail [1984, 1985a], Rosker et al. [1986], Ha et al. [1986]).

The observability of quantum beats in molecular fluorescence depends on another factor related to the theorem mentioned in section 3.4. To understand the implications of this theorem, consider the three-level model in fig. 7. The ground state $|\mathrm{g}\rangle$ is radiatively coupled to zero-order level $|s\rangle$ but not to $|r\rangle,|s\rangle$ and $|r\rangle$ are coupled by the intramolecular coupling $W$. Partial diagonalization of the

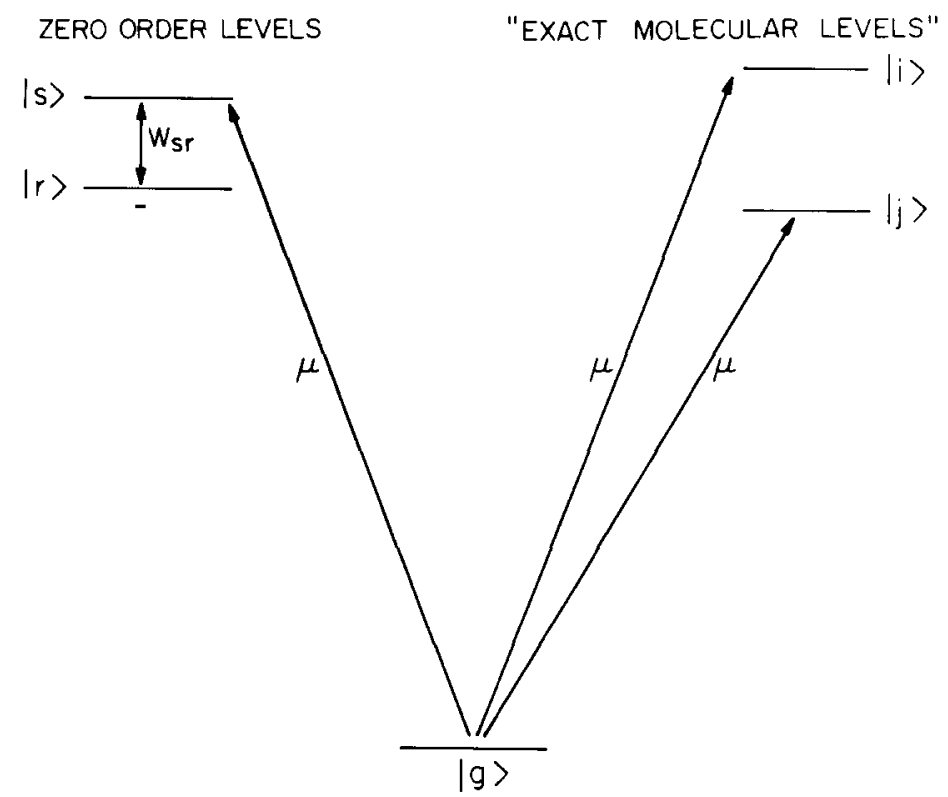

Fig. 7. A three-level model for quantum beats. (From Freed and Nitzan [1980]). 
Hamiltonian leads to exact molecular eigenlevels $|i\rangle$ and $|j\rangle$ which are linear combinations of the states $|s\rangle$ and $|r\rangle$ which diagonalize the Hamiltonian $H_{0}^{\mathrm{M}}+W$. Both $|i\rangle$ and $|j\rangle$ contain contributions from $|s\rangle$ and both are therefore coupled radiatively to $|\mathrm{g}\rangle$. We assume that the energy spacing $E_{i j}=E_{i}-E_{j}$ is large relative to the level widths so that the levels $|i\rangle$ and $|j\rangle$ can be selectively excited. A broad band, short-time pulse excitation of the system which is in $|\mathrm{g}\rangle$ initially leads to a coherent linear combination of the $|i\rangle$ and $|j\rangle$ states,

$$
|\psi(t=0)\rangle=|s\rangle=a_{s i}|i\rangle+a_{s j}|j\rangle
$$

which evolve in time according to

$$
|\psi(t)\rangle=a_{s i} \exp \left(-\mathrm{i} E_{i} t-\frac{1}{2} \gamma_{i} t\right)|i\rangle+a_{s j} \exp \left(-\mathrm{i} E_{j} t-\frac{1}{2} \gamma_{j} t\right)|j\rangle
$$

where $\gamma_{i}$ and $\gamma_{j}$ are the radiative widths of these two levels. For times longer than the inverse energy spacing $1 / E_{i j}$ the emission spectrum can be resolved; the intensity of the line at energy $E_{j g}$ is

$$
\gamma_{j} P_{j}(t)=\gamma_{j}\left|a_{s j}\right|^{2} \exp \left(-\gamma_{j} t\right)
$$

As long as these two emission components are resolved, no quantum beats are expected in the corresponding intensities.

Consider now the total integrated intensity. We have shown that this is proportional to the population of the zero-order $|s\rangle$ level, obtained by taking $n=2$ in (3.14), viz.,

$$
P_{s}(t)=|\langle s \mid \psi(t)\rangle|^{2}=\left|a_{s i}\right|^{4} \exp \left(-\gamma_{i} t\right)+\left|a_{s j}\right|^{4} \exp \left(-\gamma_{j} t\right)+2\left|a_{s i}\right|^{2}\left|a_{s j}\right|^{2} \exp \left[-\frac{1}{2}\left(\gamma_{i}+\gamma_{j}\right) t\right] \cos \left(E_{i j} t\right)
$$

Quantum beats should be observed in the integrated intensity for

$$
E_{i j}>\frac{1}{2}\left(\gamma_{i}+\gamma_{j}\right)
$$

The observation of quantum beats in fluorescence spectra depends on the mode of detection. Beats are not expected in the resolved components but, when some other conditions are fulfilled, should be observed in the total intensity of the emission which corresponds to a particular zero-order level. These beats can be suppressed by increasing spectral resolution.

\section{Vibrational energy transfer in model nonlinear oscillator chains; the Fermi-Pasta-Ulam paradox and the Kolmogorov-Arnol'd-Moser theorem}

In this and the next section, we review theories which adopt a more microscopic view of vibrational energy transfer processes. These are classical mechanical treatments which, while not strictly applicable to molecular systems away from the correspondence-principle limit, have guided subsequent developments significantly. 


\subsection{The Fermi-Pasta-Ulam (FPU) paradox}

Until the relatively recent exploration of chaotic phenomena, it was generally believed that when a large number of linear harmonic oscillators are suitably coupled by nonlinear forces, an approach to equilibrium would ensue. This belief was based on the work of Poincare $[1890,1957]$, further supported by Whittaker [1944], and Fermi's theorem [Fermi 1923; Ter Haar 1954], which stated that weakly coupled nonlinear systems will exhibit the ergodic behavior necessary for an approach to equilibrium. For a finite number of oscillators, deviations from equilibrium are expected [Mazur and Montroll 1960] and the system will return to its initial conditions because of Poincaré recurrences. However, when the number of oscillators is large, the Poincare period is presumed large [McLennan 1959] and deviations from equilibrium rare [Mazur and Montroll 1960].

Fermi, Pasta and Ulam [1955] tried to illustrate the expected approach to equilibrium by observing the equipartition of energy among normal modes of a system of one-dimensional oscillators obeying equations of the type

$$
\mathrm{d}^{2} y_{i} / \mathrm{d} t^{2}=\left(y_{i+1}-2 y_{i}+y_{i-1}\right)+\alpha\left[\left(y_{i+1}-y_{i}\right)^{2}-\left(y_{i}-y_{i-1}\right)^{2}\right],
$$

where $i$ runs over the positive integers from unity to as high as 64 . Here $\alpha$ is chosen to be sufficiently small that the nonlinear terms can be treated as a small perturbation. Using the then-new MANIAC-I computer at Los Alamos, they solved eq. (4.1) numerically and calculated the energy in each normal mode as a function of time. These calculations revealed no tendency towards equipartition of energy among the normal modes. Various initial conditions were used and various couplings were considered without changing the outcome significantly. Energy was shared by only a few modes in a periodic fashion reminiscent of the energy sharing between two vertical pendulums hanging from the same horizontal string. These results were in contradiction to the previously widely held notions concerning the approach to equilibrium.

The unexpected result of the FPU calculation was qualitatively explained by Ford [1961]: a system of harmonic oscillators (which, in this case, are the normal modes of the chain system) weakly coupled by nonlinear couplings will not achieve equipartition of energy as long as the frequencies $\omega_{k}$ are linearly independent on the integers, i.e., as long as there is no collection of integers $\left\{n_{k}\right\}$ for which

$$
\sum_{k=1}^{N-1} n_{k} \omega_{k} \cong 0
$$

other than $\left\{n_{k}\right\}=0$. These frequency matchings are known as "internal resonances" in the terminology of Kryloff and Bogoliuboff [1947], and play a crucial role in the proof of Poincarés theorem mentioned above [Poincaré 1890, 1957], as well as in Whittaker's construction [Whittaker 1944] of the constants of motion called for in Poincaré's theorem. Physically, the linear independence of uncoupled frequencies means that none of the interacting oscillators drives another at its resonance frequency, and this lack of internal resonance precludes appreciable energy sharing in the limit as the coupling tends to zero. Ford showed that for the system selected by FPU there were no such active resonances. Further (and quantitative) treatments of the FPU paradox were performed by Jackson [1963a,b] and Zabusky and Kruskal [1965]. The first explanation in terms of "overlapping resonances" appeared in Izrailev and Chirikov [1966]. 
Ford's perturbation-theoretical reasoning was further extended by Jackson [1963a,b] who was able to provide a quantitative explanation of the FPU results. It was also Jackson who pointed out that Fermi's theorem [Fermi 1924; Ter Haar 1954] was valid far less often than it was presumed at the time. Concurrently, Ford and Waters [1963] provided a computer study of energy sharing and ergodicity in coupled nonlinear oscillator systems, in which they underscored the importance of the internal resonance conditions, eq. (4.2), by building resonances into their coupled system and observing their effect on the energy sharing. The recurrences observed in the linear chain systems were analyzed and explained by Zabusky and Kruskal [1965] with their introduction of solitons [Collins 1981]. As seen before [Jackson 1963a,b] these researchers also found that integrable approximations would reproduce the FPU results, and the system was not chaotic.

Indeed, by this time, the earlier results by Kolmogorov [1954], Arnol'd [1963a,b] and Moser [1962] had begun to be appreciated. Contrary to the expectation of the appearance of statistical properties in weakly nonlinear systems, these results (subsequently known as the KAM theorem, see below) indicated that for sufficiently small perturbations, the motion of a nonlinear system retains its quasiperiodic nature. It also indicated that there would be a critical perturbation when stochasticity sets in. Such an estimate was provided by Chirikov [1960], and Izrailev and Chirikov [1966] by means of the so-called "resonance overlap" criterion. These issues will be discussed more fully by J. Ford in a review in Physics Reports.

\subsection{The Kolmogorov-Arnol'd-Moser (KAM) theorem and nonlinear resonances}

In attempting to determine the behavior of nonlinear oscillator systems governed by the Hamiltonians of the form

$$
H=H_{0}+V
$$

where $H_{0}$ represents an integrable system of oscillators and where $V$ represents a weak nonlinear and nonintegrable perturbation, most investigators had proceeded along two divergent paths. One approach assumes that the weak perturbation $V$ changes the unperturbed motion only to the extent of slightly shifting the frequencies of the motion and introducing small nonlinear harmonics. This approach is used most often when the number of oscillators is small and is exemplified in certain perturbation expansions due to Poincaré [1957], Kryloff and Bogoliuboff [1947], and Birkhoff [1927]. The second approach assumes that $V$, even though it is weak, has a profound and pathological effect on the unperturbed motion, converting it into ergodic motion. This latter approach uses the methods of statistical mechanics (presumed valid when the number of oscillators is large) and is exemplified in the work of Fermi [1923] and Peierls [1929].

A brief article by Kolmogorov [1954] enunciated a theorem which provided the cornerstone for linking these two divergent views on the effects of the weak perturbation $V$. Kolmogorov did not present a detailed proof of his theorem; the missing proof, which is quite long and mathematically sophisticated, was given almost a decade later by Arnol'd [1963a] and independently by Moser [1962]. The theorem was made known and accessible to many scientists by, among others, the work of Walker and Ford [1969] on coupled two-degree of freedom systems. And it is on these low-dimensional systems that we would like to illustrate the theorem. When action-angle variables $\left(J_{i}, \phi_{i}\right)$ are introduced for a two-oscillator system, the Hamiltonian (4.3) can be rewritten

$$
H=H_{0}\left(J_{1}, J_{2}\right)+V\left(J_{1}, J_{2}, \phi_{1}, \phi_{2}\right) .
$$


If we set $V=0$, then Hamiltonian (4.4) generates motion for which the actions $J$ are constant, and $\phi_{i}=\omega_{i}\left(J_{1}, J_{2}\right) t+\phi_{i 0}$, where the unperturbed frequencies $\omega_{i}$ are given by

$$
\omega_{i} \equiv \partial H_{0} / \partial J_{i}
$$

Following Kolmogorov, we view the unperturbed motion in phase space as lying on two-dimensional tori where $\left(\phi_{1}, \phi_{2}\right)$ are the angle coordinates on the torus and $\left(J_{1}, J_{2}\right)$ are the radii of the tori. By assuming that $V$ is sufficiently small and by assuming that the Jacobian of the frequencies

$$
\partial\left(\omega_{1}, \omega_{2}\right) / \partial\left(J_{1}, J_{2}\right) \neq 0
$$

Kolmogorov, Arnol'd and Moser (KAM) were able to show that most of the unperturbed tori bearing conditionally periodic motion with incommensurate frequencies continue to exist, being only slightly distorted by the perturbation. On the other hand, the tori bearing periodic motion, or very nearly periodic motion, with commensurate frequencies, or with incommensurate frequencies whose ratio is approximated very well by $(r / s)$ where $r$ and $s$ are relatively small integers, are grossly deformed by the perturbation and remain no longer close to the unperturbed tori. Since the unperturbed tori with commensurate frequencies which are destroyed by the perturbation are dense everywhere, it is remarkable that KAM are able to show that the majority (in the sense of measure theory) of initial conditions for Hamiltonian (4.3) lie on the preserved tori bearing conditionally periodic motion when $V$ is sufficiently small.

Thus for small $V$, the KAM theorem says that for most initial conditions Hamiltonian (4.3) generates nonergodic motion. This justifies the view that the perturbation $V$ largely serves only to change the frequencies slightly and to introduce small nonlinear harmonics into the motion. Nevertheless, the relatively small set of initial conditions leading to motion not on preserved tori are, from a physical point of view, pathologically interspersed between the preserved tori. Thus, if Hamiltonian (4.4) is ever to provide generally ergodic motion best described in terms of statistical mechanics, the source of such behavior must lie in the reasons for the very existence of this relatively small set of destroyed tori.

\subsection{The onset of stochasticity}

As far as intramolecular energy transfer is concerned, it would be very desirable to have practical guidelines for the onset of instability in coupled oscillator systems. With this practical aim in mind, as well as preparation for later applications, we will follow the lead of Walker and Ford [1969] and concentrate on analyzing the onset of ergodic behavior in a two-degree of freedom oscillator system.

Let us expand the $V$ of Hamiltonian (4.4) in a Fourier series and write

$$
H=H_{0}\left(J_{1}, J_{2}\right)+f_{m n}\left(J_{1}, J_{2}\right) \cos \left(m \phi_{1}+n \phi_{2}\right)+\cdots,
$$

where we have explicitly written only one term in the series. The KAM formalism seeks to eliminate the angle-dependent terms using a convergent sequence of canonical transformations, each of which is close to the identity transformation, thus obtaining a Hamiltonian which is a function of the transformed action variables alone and is close to the initial Hamiltonian (for explicit implementations in the chemical physics literature, see Chapman et al. [1976], Swimm and Delos [1979], Jaffé and Reinhardt [1982]). If this can be accomplished in some general sense, then one immediately finds that the perturbed motions lie, for the most part, on tori close to the unperturbed tori. 
As an example, let us eliminate the explicit angle-dependent term in the Hamiltonian (4.7) by introducing the canonical transformation generated by [Goldstein 1980]

$$
F_{2}=J_{1}^{\prime} \phi_{1}+J_{2}^{\prime} \phi_{2}+B_{m n}\left(J_{1}^{\prime}, J_{2}^{\prime}\right) \sin \left(m \phi_{1}+n \phi_{2}\right)
$$

where $\left(J_{1}^{\prime}, J_{2}^{\prime}\right)$ are transformed action variables to be determined. We note that if $B_{m n}=0$, we have the identity transformation. Introducing the canonical transformation generated by (4.8) into Hamiltonian (4.7), we obtain

$$
\begin{aligned}
& H=H_{0}\left(J_{1}^{\prime}, J_{2}^{\prime}\right)+\left\{\left[m \omega_{1}\left(J_{1}^{\prime}, J_{2}^{\prime}\right)+n \omega_{2}\left(J_{1}^{\prime}, J_{2}^{\prime}\right)\right] B_{m n}\left(J_{1}^{\prime}, J_{2}^{\prime}\right)+f_{m n}\left(J_{1}^{\prime}, J_{2}^{\prime}\right)\right\} \cos \left(m \phi_{1}+n \phi_{2}\right)+\cdots, \\
& \omega_{i}\left(J_{1}^{\prime}, J_{2}^{\prime}\right)=\partial H_{0}\left(J_{1}^{\prime}, J_{2}^{\prime}\right) / \partial J_{i} .
\end{aligned}
$$

We may eliminate the given angle-dependent term by setting

$$
B_{m n}\left(J_{1}^{\prime}, J_{2}^{\prime}\right)=-\frac{f_{m n}\left(J_{1}^{\prime}, J_{2}^{\prime}\right)}{m \omega_{1}\left(J_{1}^{\prime} ; f_{2}^{\prime}\right)+n \omega_{2}\left(J_{1}^{\prime}, J_{2}^{\prime}\right)},
$$

and provide that the denominator in eq. (4.10) is not zero or very small compared with $f_{m n}$. If it is very small, the coefficient $B$ is large, the transformation generated in eq. (4.7) is not close to the identity transformation, and the transformed coordinate motion is not close to the unperturbed motion. As a consequence, if there exists a band of frequencies $\omega_{i}$ for the Hamiltonian (4.4) satisfying

$$
\left|m \omega_{1}\left(J_{1}, J_{2}\right)+n \omega_{2}\left(J_{1}, J_{2}\right)\right| \ll\left|f_{m n}\left(J_{1}, J_{2}\right)\right|,
$$

then the angle-dependent term $\cos \left(m \phi_{1}+n \phi_{2}\right)$ grossly distorts an associated zone of unperturbed tori bearing the frequencies satisfying the inequality (4.11).

Moreover, when a zone of unperturbed tori is grossly distorted by a specified angle-dependent term $\cos \left(m \phi_{1}+n \phi_{2}\right)$, one must in general anticipate that there will be a host of angle-dependent terms $\cos \left(m^{\prime} \phi_{1}+n^{\prime} \phi_{2}\right)$ in Hamiltonian (4.4) whose $m^{\prime} / n^{\prime}$ ratios are sufficiently close to the specified ratio $m / n$ that the analog of inequality (4.11) is satisfied for them also. Hence the zone of unperturbed tori distorted by $\cos \left(m \phi_{1}+n \phi_{2}\right)$ will simultaneously be affected by a large number of other angledependent terms. Physically speaking, inequality (4.11) is a resonance relationship which, if satisfied, asserts that $\cos \left(m \phi_{1}+n \phi_{2}\right)$ resonantly couples the oscillators when their frequencies lie in the designated band. If a number of angle-dependent resonant terms couple the oscillators in this band, then one has the situation envisioned in the quantum-mechanical Golden Rule in which an initial state is resonantly coupled to a density of final states leading to statistically irreversible behavior. In analogy, one would anticipate that the motion generated by Hamiltonian (4.4) in the overlapping resonant zones of destroyed tori is highly complicated and perhaps ergodic.

When $V$ is very small and hence all $f_{m n}$ are small, the inequality (4.11) shows that the resonance zones are very narrow. Moreover, KAM show that the totality of all resonant destroyed zones is small relative to the measure of the allowed phase space [Rosenbluth et al. 1966]. However, as the $V$ and the $f_{m n}$ increase, or equivalently as the total energy increases, one anticipates from inequality (4.11) that the measure of the overlapping resonant zones may increase until most of phase space is filled with highly complicated trajectories moving under the influence of many resonances. In short, the KAM 
theory indicates the existence of an amplitude instability for conservative nonlinear systems which permits a transition from motion which is predominantly conditionally periodic to motion which is predominantly ergodic. The Walker and Ford paper [1969] establishes, in pictorial form, this transition and makes the influence of resonance zone overlap evident [Ford 1978; Berry 1978]; for the terminology, see Schewe and Gollub [1985]. For the transition from classical mechanics to statistical mechanics, see Ford [1975]; also Tabor [1981, 1989].

For systems with three or more degrees of freedom, the approach to stochasticity differs fundamentally from the preceding example [Lichtenberg and Lieberman 1983]. Briefly, resonances between oscillators in two degrees of freedom lead to the formation of resonance layers each of which plays host to stochastic motion. However, energy conservation prevents large excursions of the motion along the layer - only motion across the layer is important. For near-integrable systems with a weak perturbation, the stochastic layers are isolated by KAM surfaces. However, this is not true for resonance layers in three or more degrees of freedom. Instead, they intersect, forming a connected web dense in action space (for an illustration, see Tennyson et al. [1980]). Also, conservation of energy no longer prevents large stochastic motions of the actions along layers over long times. The system can therefore explore all regions of phase space consistent with energy conservation by stepping from layer to layer in this interconnected dense set of layers. The basic mechanism of diffusion along resonance layers is called Arnold diffusion [Arnold 1963b]. While there is no critical perturbation strength for its presence, Arnold diffusion is known to vanish with vanishing perturbation strength.

One of the first numerical demonstrations of an amplitude instability was made in an investigation of unimolecular dissociation by the chemical physicists Thiele and Wilson [1961] and Bunker [1962] (see the review by Hase [1976]). These investigators noted that for small-amplitude motion, the harmonic modes of model triatomic molecules exhibited very little mode-mode energy transfer. As the energy of the molecule was increased, an amplitude stability set in which allowed free and rapid interchange of energy between harmonic modes. Consequently, as the energy was further increased to slightly above that needed to dissociate one atom from the molecule, almost all initial configurations led to dissociation. Thiele and Wilson [1961], who were using coupled Morse oscillators, then used these results to argue that nonlinearity must be given a central role in developing a statistical theory of unimolecular reactions. While these molecular systems were not analyzed in terms of the KAM theorem, Walker and Ford have shown [1969] that the instability observed by Thiele and Wilson [1961] occurs concurrently with large-scale disappearance of preserved tori.

The second major numerical demonstration of amplitude instability occurred in an astronomical system, when Hénon and Heiles [1964] studied the Hamiltonian

$$
H=\frac{1}{2}\left(p_{1}^{2}+q_{1}^{2}+p_{2}^{2}+q_{2}^{2}\right)+q_{1}^{2} q_{2}-\frac{1}{3} q_{2}^{3},
$$

which now bears their name. This Hamiltonian has made frequent appearances in the theory of molecular energy transfer where it describes a molecular potential with a $\mathrm{C}_{3 v}$ symmetry. Another popular system for demonstrating KAM instability is the Hamiltonian

$$
H=\frac{1}{2} \sum_{k}\left(P_{k}^{2}+\omega_{k}^{2} q_{k}^{2}\right)+\alpha \sum_{i, j, k} A_{i j k} q_{i} q_{j} q_{k}+\lambda \sum_{h, i, j, k} B_{h i j k} q_{h} q_{i} q_{j} q_{k}
$$

The studies by FPU [1955], Ford and Waters [1963], Jackson [1963a,b] and Zabusky and Kruskal [1965] were done on Hamiltonians of this form. But Izrailev and Chirikov [1969] were the first to suggest that Hamiltonian (4.13) should exhibit a KAM instability leading to statistical behavior. 
Many criteria have been proposed for the determination of the transition to global stochasticity [Chirikov 1960, 1979; Izrailev and Chirikov 1969; Jaeger and Lichtenberg 1972; Greene 1968, 1979; Escande and Doveil 1981; Percival 1974]. For a summary, see Lichtenberg and Lieberman [1983]; the subject has recently been reviewed by Escande [1985]. The earliest criterion, due to Chirikov [1960], is known as the "resonance overlap" criterion, and constitutes an approximate yet elegant and powerful intuitive device for understanding the physics of this transition. In its simplest form, it postulates that the last KAM surface between two lowest-order resonances is destroyed when the sum of the half-widths of their island separatrices (see section 5.1) formed by them (but calculated independently of each other) equals the distance between the resonances. The distances are measured either in frequency or action space, whichever is more convenient. Because of its highly practical prescription, this criterion has been widely used.

The effect of resonance overlap was exhibited by Ford and Lunsford [1979] on a multiple-oscillator system with very small nonlinear couplings. Among the numerous recent implementations of the "overlap of resonances" criterion are those of Rechester and Stix [1979], and Petrosky [1984]. Resonance overlap can occur for these systems in the limit of arbitrarily small couplings when the frequencies of the system fulfill commensurability conditions which allow the nonlinear couplings to couple all internal degrees of freedom. The stability of the periodic orbits for the Henon-Heiles oscillator was studied by Lunsford and Ford [1972], who were able to demonstrate the existence of a dense or nearly dense set of unstable periodic orbits throughout its stochastic (unstable) regions of phase space. They also showed the intimate relation between the Henon-Heiles system, the FPU system as well as the so-called Toda lattice [Lichtenberg and Lieberman 1983].

Energy transfer processes in nonlinearly coupled chains and, in particular, the approach to statistical equilibrium in such systems is still a subject of interest and research. For example, see Henry and Oitmaa [1983, 1985a,b], Florencio and Lee [1985], Henry and Grindlay [1986, 1987, 1988].

\section{Classical microscopic theory of energy sharing; energy transfer through nonlinear resonances}

In the previous section we have seen the crucial role that nonlinear (or "internal") resonances play in causing instabilities in dynamical systems. The effect of resonances had been appreciated by molecular physicists before the study of intramolecular energy transfer since resonances showed up in the distortion of vibrational spectra. In fact, the splitting of the almost degenerate vibrational energy levels in the carbon dioxide molecule was explained by Fermi [1931] as being due to an almost exact $2: 1$ frequency resonance between the bending and the stretching modes of this molecule (for more recent treatments of the quantization and dynamics, see Heller et al. [1979, 1980], Noid et al. [1979], Sibert et al. [1983], Uzer [1984], Brabham and Perry [1984], Voth et al. [1984], Peyerimhoff et al. [1984], Voth and Marcus [1985], Kato [1985b], Baggott et al. [1985, 1986], Farrelly [1986a], Farrelly and Uzer [1986], Kellman and Lynch [1986a,b, 1988a,b], Martens and Ezra [1987a,b], Green et al. [1987], Wong et al. [1987], Quapp and Heidrich [1988], Quapp [1989], Colbert and Sibert [1989]).

Since these frequency resonances are central to both the dynamical and static properties of molecules, we will present the classical and quantum mechanics of energy transfer through a resonance. In reviewing this field, our task is considerably eased by the existence of several informative and comprehensive reviews and monographs: Ford [1973], Rice [1981], as well as Brumer [1981] and Tabor $[1981,1989]$, where the emphasis is on the onset of statistical behavior. Rather than recounting the material and the numerous references contained in these reviews, we will focus on developments of the intervening decade. 
As an aside, much effort in chemical physics has gone into the related but distinct subject of semiclassical quantization of nonlinear dynamical systems (e.g., Eastes and Marcus [1974], Noid and Marcus [1975], Sorbie and Handy [1976], Chapman et al. [1976], Swimm and Delos [1979], Jaffé and Reinhardt [1982], Farrelly and Smith [1986], Farrelly [1986b], Ratner et al. [1987], Taylor and Grozdanov [1987], Gerber and Ratner [1988], De Leon and Mehta [1988], Kummer and Gompa [1988], Sibert [1988]). Comprehensive reviews on both general and special procedures are available (Noid et al. [1981a,b], Reinhardt [1982], Delos [1986], Bowman [1986], Ezra et al. [1987], Reinhardt and Dana [1987], Skodje and Cary [1988], Reinhardt [1989]).

\subsection{Nonlinear resonances and the classical mechanics of the pendulum}

Like many other applications of nonlinear dynamics in molecular physics, the pendulum or the hindered rotor picture originated in plasma physics research. This paradigmatic picture was recognized and developed [Chirikov 1959; Zaslavskii and Filonenko 1968] to understand the trapping of charged particles. Soon it was realized that this picture could be applied generally to a number of other trapping phenomena, including trapping and leakage in phase space. For an extensive review of the derivation of the pendulum picture the reader is referred to Chirikov [1979], as well as to standard texts [Lichtenberg and Lieberman 1983, Zaslavsky 1985]. For our purposes, the following brief derivation (see section 4.3) will suffice [Brumer 1981].

Suppose one is considering the dynamics of a coupled oscillator system, the zero-order frequencies of which have a resonance, i.e. a commensurability relation, like eq. (4.2). It is convenient and customary to analyze the nonlinear coupling in terms of the action-angle variables of the unperturbed system, $(J, \phi)$,

$$
H(J, \boldsymbol{\phi})=H_{0}(J)+\sum_{\{m\}} V_{\{m\}}(J) \exp (\mathrm{i} \boldsymbol{m} \cdot \boldsymbol{\phi})
$$

where $\boldsymbol{m}$ is a multidimensional vector of integers. In particular, for two coupled oscillators,

$$
H(J, \boldsymbol{\phi})=H_{0}(J)+\sum_{m_{1}, m_{2}} V_{m_{1}, m_{2}}(J) \exp \left[\mathrm{i}\left(m_{1} \phi_{1}+m_{2} \phi_{2}\right)\right]
$$

or concentrating on a single resonance,

$$
H(\boldsymbol{J}, \boldsymbol{\phi}) \simeq H_{0}(J)+V_{m_{1}, m_{2}}(J) \exp \left[\mathrm{i}\left(m_{1} \phi_{1}+m_{2} \phi_{2}\right)\right]
$$

If the system contains a resonance,

$$
m_{1} \omega_{1}(J)+m_{2} \omega_{2}(J)=0
$$

for a particular set of action $\boldsymbol{J}^{\mathrm{r}}$, then terms like $\exp \left[\mathrm{i}\left(m_{1} \phi_{1}+m_{2} \phi_{2}\right)\right]$ are slowly varying in time (cf. the small-denominator problem in the preceding section). Introducing the new independent angle variables,

$$
\psi_{k}=\sum_{i=1}^{2} \mu_{k i} \phi_{i} \quad(k=1,2)
$$

where $\mu_{k i}$ are constants such that $\mu_{11}=m_{1}, \mu_{12}=m_{2}, \mu_{21}=m_{1}, \mu_{22}=m_{2}$. The new actions $I$ are 
obtained by the generating function

$$
F_{2}(\boldsymbol{I}, \boldsymbol{\phi})=\sum_{i=1}^{2}\left(I_{i}^{\mathrm{r}}+\sum_{k=1}^{2} I_{k} \mu_{k i}\right) \phi_{1}
$$

giving

$$
I_{k}=\sum_{i}\left(I_{i}-I_{i}^{\mathrm{r}}\right) \mu_{i k}^{-1}
$$

where $\mu_{i k}^{-1}$ are matrix elements of the matrix $\boldsymbol{\mu}^{-1}$.

The new momenta $I$ measure deviations from $I^{r}$. Since $\psi_{2}$ does not occur in the resonance Hamiltonian (5.3), $I_{2}$ is a constant of the motion, and (5.3) can be rewritten

$$
H_{\mathrm{r}}(\boldsymbol{I}, \boldsymbol{\psi})=\sum_{i, k} I_{k} \mu_{k \iota} \omega_{i}+\sum_{k, l} \frac{I_{k} I_{l}}{2 M_{k l}}+V_{m_{\mathrm{l}}, m_{2}}(I) \cos \psi_{1} .
$$

Here, $M_{k l}=\Sigma_{i, j}^{2} \mu_{k i}\left(\partial \omega_{i} / \partial I_{j}\right) \mu_{l j}$ is a generalized mass.

At the resonance center, (5.4) can be applied, and (5.8) becomes

$$
H_{\mathrm{r}}(\boldsymbol{I}, \boldsymbol{\psi})=\left(1 / 2 M_{11}\right) I_{1}^{2}+V_{m_{1}, m_{2}}(\boldsymbol{I}) \cos \psi_{1}
$$

The approximation to the dynamics has been obtained by averaging over the fast motion (see also Born [1960], Kryloff and Bogoliuboff [1947]), leaving only the slowly varying terms. Therefore, this process has resulted in a lower-dimensional "resonance Hamiltonian" $H_{r}$, eq. (5.9), which can be examined for some of the salient features of the original Hamiltonian.

Special cases arise when the anharmonicity disappears, i.e., the system becomes "intrinsically degenerate" [Izrailev 1980; Martens and Ezra 1987b] as opposed to accidentally degenerate, which is the case we treat. Often one can approximate the Fourier coefficients at the center of the resonance, i.e., when condition (5.4) is fulfilled.

While it is not necessary to make this approximation for subsequent analysis, it simplifies the mathematics and sometimes is accurate (for semiclassical evalution of matrix elements, see Noid et al. [1977], Koszykowski et al. [1982a], Wardlaw et al. [1984], Ozorio de Almeida [1984a], Voth and Marcus [1985], Voth [1986], Noid and Gray [1988]). Examples of allowing the Fourier coefficients to vary are to be found in Gray and Child [1984], Shirts [1987a], Sahm and Uzer [1989a], Sahm et al. [1989].

The resulting approximate Hamiltonian (the subscripts are not needed),

$$
H_{\mathrm{r}}(I, \psi)=(1 / 2 M) I^{2}+V\left(I^{\mathrm{r}}\right) \cos \psi=E_{\mathrm{r}},
$$

is that of a pendulum, or a hindered rotor (for an application of this procedure to two coupled Morse oscillators, see Jaffé and Brumer [1980]). It is clearly an integrable system, and it has three types of motion. When the energy is less than the potential barrier,

$$
E_{\mathrm{r}}<V\left(I^{\mathrm{r}}\right)
$$

the motion is that of "libration". When the energy is above the barrier, it is "rotation" [Noid and 
Marcus 1977]. These terms originate from the motion of a physical pendulum, but are commonly used when the pendulum variables are abstract quantities, as well. The third type of motion is the motion on the separatrix, i.e., when the energy is precisely equal to the barrier height. The phase space profile of these three motions is shown in fig. 8. The pendulum is such a useful system that we quote the results of its analysis in full. Its action-angle variables are given by Zaslavskii and Filonenko [1968], Rechester and Stix [1979], Lieberman and Lichtenberg [1984],

$$
\begin{aligned}
& J=(8 / \pi)(V M)^{1 / 2} \times \begin{cases}E(\kappa)-\left(1-\kappa^{2}\right) K(\kappa), & \kappa<1, \\
\frac{1}{2} \kappa E\left(\kappa^{-1}\right), & \kappa>1,\end{cases} \\
& \theta=\frac{1}{2} \pi \times \begin{cases}{[K(\kappa)]^{-1} F(\eta, \kappa),} & \kappa<1, \\
2\left[K\left(\kappa^{-1}\right)\right]^{-1} F\left(\psi / 2, \kappa^{-1}\right), & \kappa>1,\end{cases} \\
& 2 k^{2}=1+E_{\mathrm{r}} / V,
\end{aligned}
$$

where $K, E$ are complete elliptic integrals of the first and second kinds, $F$ is an incomplete elliptic integral of the first kind, and

$$
\kappa \sin \eta=\sin (\psi / 2) .
$$

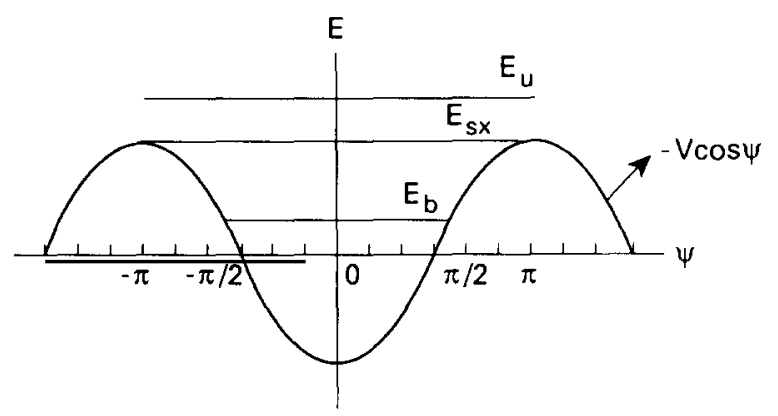

(a)

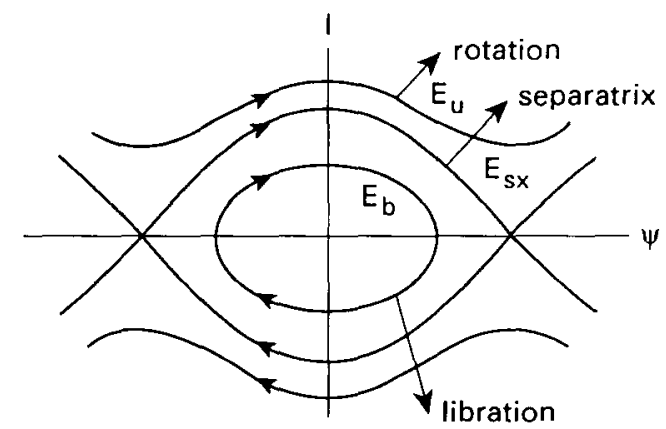

(b)

Fig. 8. Correspondence between a, an energy diagram, and $b$, a phase space diagram for the pendulum Hamiltonian. (From Lichtenberg and Lieberman [1983]). 
The approximate width of the resonance in action and angle, $(\Delta I)^{\mathrm{r}}$ and $(\Delta W)^{\mathrm{r}}$, respectively, is given by Zaslavskii and Chirikov [1972], Shuryak [1976], Brumer [1981] [in terms of the original variables of eq. (5.9)],

$$
(\Delta J)^{\mathrm{r}}=2 \boldsymbol{m}\left(M_{11} V_{m_{1}, m_{2}}\right)^{1 / 2}, \quad(\Delta W)^{\mathrm{r}}=\frac{2}{|\boldsymbol{m}|}\left(V_{m_{1}, m_{2}} / M_{11}\right)^{1 / 2},
$$

where $\boldsymbol{m}=\left(m_{1}, m_{2}\right)$. The explicit time dependence of the variables, on the other hand is [Zaslavskii and Filonenko 1968]

$$
I(t)=2 \omega_{0} \kappa \operatorname{cn}\left(\omega_{0} t\right), \quad \kappa<1 ; \quad I(t)=2 \omega_{0} \kappa \operatorname{dn}\left(\omega_{0} t\right), \quad \kappa>1,
$$

where $\omega_{0}=(V / M)^{1 / 2}$ and the frequency of the pendulum is

$$
\omega(\kappa) / \omega_{0}=\frac{1}{2} \pi[K(\kappa)]^{-1}, \quad \kappa<1 ; \quad \omega(\kappa) / \omega_{0}=\pi \kappa / K\left(\kappa^{-1}\right), \quad \kappa>1 .
$$

Near the separatrix

$$
\lim _{\kappa \rightarrow 1}\left(\omega / \omega_{0}\right)= \begin{cases}\frac{1}{2} \pi / \ln \left[4\left(1-\kappa^{2}\right)^{-1 / 2}\right], & \kappa<1, \\ \pi / \ln \left[4\left(\kappa^{2}-1\right)^{-1 / 2}\right], & \kappa>1 .\end{cases}
$$

The behavior of $\omega / \omega_{0}$ with varying $\kappa$ is plotted in Lichtenberg and Lieberman [1963], fig. 2.2.

\subsection{Vibrational energy transfer and overlapping resonances}

In section 4.3 , we have seen a classical-mechanical demonstration of the appearance of a dynamical instability due to overlap of nonlinear resonance zones (the quantal interaction of nonlinear resonances can be found, among others, in Shuryak [1976], and Berman et al. [1981]). The appreciation of the effect of nonlinear resonances in intramolecular energy transfer processes can be said to have been phrased in these terms first by Oxtoby and Rice [1976], who computed regions of resonance overlap in phase space for several coupled oscillator systems in order to correlate the extent of intramolecular energy transfer with the fraction of phase space displaying overlapping resonances. Nonlinear resonances, the pendulum picture, and the overlap of resonances criterion was also used in the early work of Kay [1980]. Later applications were very much accelerated by the study of local modes (for a review, see Child and Halonen [1984]), where it could be assumed that the initial excitation was indeed localized in one identifiable oscillator in the molecule, usually consisting of a hydrogen atom bound to a carbon or oxygen atom, which is too heavy to participate extensively in the (highly anharmonic, i.e., nonlinear) vibrations of its light partner. It is well known that the vibrations of local modes can be approximated by Morse oscillators (Child and Halonen [1984]), and therefore, most of the early theoretical applications have been done on coupled Morse oscillators [Kay 1980; Jaffé and Brumer 1980], the simplest such system being the nonrotating water molecule consisting of two $\mathrm{OH}$ stretching bending modes and a frozen bending angle [Sibert et al. 1982a,b]. For an overview of calculations concerning localization of energy, see Reinhardt and Duneczky [1988].

Jaffé and Brumer [1980], and Sibert et al. [1982a,b] studied the classical and quantal dynamics of local modes in the water molecule by means of the pendulum picture, and provided a pictorial explanation of local-mode dynamics. They realized that the energy transfer dynamics could be 
approximated very compactly by the time dynamics of the pendulum variables; indeed, the canonical transformation (5.6) in this case reduces the original Hamiltonian to one for the variable action

$$
I=I_{1}-I_{2}
$$

which, when multiplied by a representative, average frequency, approximates the energy difference between the two stretching motions. In this picture, the action of the nonlinear resonance as a self-regulating energy transfer mechanism is particularly obvious. A frequency matching is required to drive the mode-mode energy transfer; on the other hand, when energy is transferred, the frequencies change and detune from each other, causing the energy flow to stop. The pendulum picture also provides a dynamical view of the local versus normal mode classification in molecules. Local modes correspond to the rotating solution (5.12b), and normal modes to the librating solution (5.12a). The original analysis was performed in local-mode actions, and the alternative, normal-mode based description was provided by Kellman [1986].

Sibert et al. [1982b] used a one-dimensional integrable approximation to the original Hamiltonian to find the quantum-mechanical local-mode energy levels. When the action variables are replaced by $I \rightarrow(\hbar / \mathrm{i}) \mathrm{d} / \mathrm{d} \phi$, in accordance with the traditional recipe of obtaining quantum-mechanical operators from classical-mechanical Hamiltonians (see e.g., Augustin and Rabitz [1979]), a Mathieu differential equation [McLachlan 1964] results, the eigenvalues of which approximate the locations of the quantized energy levels of the water molecule rather well. Moreover, the periods found by Sibert et al. [1982b] for the energy transfer functions turned out to be related (through the uncertainty relation) to the splittings between adjacent quantized local modes, as would be expected [Lawton and Child 1979, 1981].

The quantization of a nonlinear resonance in nonautonomous systems had also led to the Mathieu equation [Berman and Zaslavskii 1977], and uniform quantization schemes [Uzer et al. 1983; Ozorio de Almeida 1984b; Uzer and Marcus-1984; Voth and Marcus 1985; Farrelly and Uzer 1986; Voth 1986; Graffi et al. 1987, 1988] have also been used to understand avoided crossings in nonlinear systems in terms of signatures of resonances [Noid et al. 1980, 1983; Ramaswamy and Marcus 1981]. For experimental observation of quantal state structure near a resonance, see Holtzclaw and Pratt [1986] and Frederick et al. [1988].

This microscopic formulation was soon extended to a more complicated system, namely the transfer of energy out of local $\mathrm{CH}$ oscillators into the vibrations of frame of the benzene molecule [Sibert et al. 1983, 1984a,b]. In this case, a simple analysis shows that the relaxation should be most effective when there is a Fermi (i.e., 2:1) resonance between the $\mathrm{CH}$ stretching and HCC bending (so-called "wagging") motions [Sibert et al. 1983]. After reaching the wagging motion, vibrational energy disperses into the numerous vibrations of the frame principally through $1: 1$ resonances. These conclusions were first reached by means of classical trajectory calculations [Sibert et al. 1984a] by analyzing the widths of resonances at each step of the relaxation process. For an experimental analysis of $\mathrm{CH}$ fundamental relaxation, see Stewart and McDonald [1983].

Classical (or quasiclassical) trajectory calculations, which are reviewed, among others, by Hase [1976], and Raff and Thompson [1985], are the most widely used procedure for studying the classical mechanical energy flow in molecular systems. Their applications to realistic potential energy surfaces, and the degree to which they can simulate the true dynamics faithfully have been discussed [Hase 1982; Hase and Buckowski 1982; Hase 1986]. The basis for the validity of classical trajectory calculations in the chaotic regime is related to the so-called $\beta$-shadow theorem (e.g., Hammel et al. [1987]). One of the persistent practical difficulties in simulating vibrations has been the contribution of zero-point 
energy to the energy flow and reactivity. The amount of zero-point energy contained in the numerous modes of a polyatomic molecule can be considerable. Recent research [Bowman et al. 1989; Miller et al. 1989] addresses this problem with schemes to prevent classical oscillator energies from falling below their zero-point energy.

In their quantum-mechanical work on benzene, Sibert et al. [1984b] used "tiers" of coupled states in very much the same way that individual quantum states were used in the fluorescent calculation in the first section of this review (IVR processes in benzene have been reviewed by Riedle et al. [1984]). The time-dependent probability flow between these states was computed and the times for coarse-grained probability transfer were then converted to lifetimes and linewidths for given initial excitations. The most striking conclusion of these calculations was an explanation of the unexpected decrease in linewidth of benzene overtone vibrations as the energy is increased (recent experiments are Perry and Zewail [1984], Page et al. [1987, 1988]; recent theory by Lu and Hase [1988]). Since the density of states increases with increasing energy, one would expect the widths to increase. On the other hand, by the time one reaches those high overtones, the system has moved out of the widths of these essential resonances, thereby slowing down energy transfer considerably. Here is a case where the density of states, which is commonly such a paramount factor in driving energy relaxation, is secondary to what could be called the microscopic detail, namely the coupling and the resonances between modes (for alternative treatments, see Stone et al. [1981], Prasad [1981], Buch et al. [1984], Mukamel and Islampour [1984], Shi and Miller [1985], Dietz and Fischer [1987], and Voth [1988]).

The work of Sibert et al. [1984a,b] on benzene and its derivatives has been followed by a considerable number of other classical and semiclassical calculations employing increasingly refined models - sometimes in the spirit of "reduced dimensionality" models (reviewed by Bowman [1985]). Refinements introduced by Lu et al. [1986], and Garcia-Ayllon et al. [1988], include, for instance, the attenuation of the bending force constant with the stretching vibrations [Swamy and Hase 1986; Wolf et al. [1986] and quasiclassical prescriptions for calculating the lineshape and width [Lu and Hase 1988]. The calculations of Bintz et al. [1986a,b; 1987] as well as Lu and Hase [1988], Clarke and Collins [1987a,b] and Garcia-Ayllon et al. [1988] have tended to affirm the mechanism initially proposed by Sibert et al. [1984a,b].

Parallel calculations on vibrational energy redistribution in other hydrocarbons [Hutchinson et al. 1983, 1984, 1986] (experiment done by Nesbitt and Leone [1982]), concluded that the energy transfer was driven mainly by $1: 1$ resonances, or combination resonances [Hofmann et al. 1988], whereas some researchers have reported that a similar resonance picture is only loosely applicable [Sumpter and Thompson 1987b] or inapplicable in their modeling of energy transfer in alkanes [Duneczky and Reinhardt 1990]. The tier-by-tier energy flow picture has been applied to IVR in highly excited acetylene and its isotopic variants, and the connection between energy flow pathways and resulting "clump" spectra has been explored by Holme and Levine [1988a,b; 1989]. For recent experiments on energy flow in alkanes following $\mathrm{CH}$ overtone excitation, see McIlroy and Nesbitt [1989]. For other chains, see, e.g., Shin [1989].

While in the preceding calculations the energy exchange among modes proceeds through potential and/or kinetic couplings, some recent work has examined the interactions of vibrations with rotations [Frederick and McClelland 1986a,b; Ezra 1986; Shirts 1986, 1987b; Lawrance and Knight 1988; Knight 1988a,b] (see also the monograph by Ezra [1982]) and internal rotations [Sumpter et al. 1988], vibration-rotation energy flow [Frederick et al. 1985; Ezra 1986; McClelland et al. 1988] and intramolecular vibration mixing induced by rotational (Coriolis) couplings [Clodius and Shirts 1984; Uzer et al. 1985a; Burleigh et al. 1988; Lin 1988; Gray and Davis 1989; Sibert 1989; Sahm and Uzer 
1989a,b; Sahm et al. 1989; Parson 1989a,b]. For recent experimental studies, see Garland et al. [1983], Watanabe et al. [1983], Nathanson and McClelland [1984], Dai et al. [1985a,b], Felker and Zewail [1985c], Amirav and Jortner [1986], Apel and Lee [1986], Garland and Lee [1986], Bain et al. [1986], Ohta et al. [1987], De Souza et al. [1988], Neusser et al. [1988], Lawrance and Knight [1988], Frye et al. [1988].

Both rotation-induced vibration mixing and vibration-induced rotation mixing have been examined in a two-mode model of the formaldehyde molecule [Burleigh et al. 1988; Sibert 1989; Gray and Davis 1989] and its deuterated analog [Parson 1989, 1990]. These investigations show the consequences of the collective operation of these effects. They also suggest that in regions of experimental interest, the intramolecular dynamics is not significantly influenced by resonances. An examination of vibrational energy flow under the influence of both Coriolis and potential couplings [Fischer and Dietz 1987; Hiroike and Fujimura 1988; Burleigh et al. 1988; Sahm and Uzer 1989a,b] has led to the development of a picture of the joint action of rotational and potential couplings [Sahm and Uzer 1989a,b; Sahm et al. 1989], see also next section. Vibrations and rotations interact through both Coriolis and centrifugal couplings. The relative roles of these mechanisms have been discussed in Lawrance and Knight [1988], and reviewed in Knight [1988a,b]. For a recent rovibronic IVR model calculation, see Dietz and Fischer [1987].

Classical and/or quantal propagation methods have been used to track energy flow through potential energy blockers [Uzer and Hynes 1986], for example, a heavy metal atom [Lopez and Marcus 1982; Swamy and Hase 1985; Lederman et al. 1986; Lopez et al. 1986; Uzer and Hynes 1987; Lederman et al. 1989; Uzer and Hynes 1989]. Experimentally the possibility seems to exist [Rogers et al. 1982, 1983] that energy may be localized in one reactive portion of an energized molecule by a heavy metal atom; RRKM behavior involving rapid redistribution and reaction remote from the bond excitation site is thwarted, though statistical behavior has been reported by Wrigley et al. [1983, 1984] in some molecules with heavy metal "blockers". The possibility of these blockers inducing nonstatistical reactive behavior is discussed by Uzer and Hynes [1986, 1989].

The preceding calculations are primarly (but not exclusively) concerned with vibrational energy redistribution leading to relaxation. There are other calculations in which the redistribution of energy can lead to unimolecular reaction (e.g. Rai and Kay [1984], Uzer [1988]). While such calculations, starting with Thiele and Wilson [1961] and the extensive work of Chapman, Bunker, Hase, Thompson and colleagues (for a review of their work, see Hase [1976, 1981], Raff and Thompson [1985], Hase [1986]), have commonly simulated reagents that develop from collisions (e.g., Viswanathan et al. [1984, 1985], NoorBatcha et al. [1986], Rice et al. [1986]) or chemical activation (Wolf and Hase [1980a], Hase et al. [1983, 1984]), more recent examples have probed the consequences of modespecific excitation [Sloane and Hase 1977, Marcus et al. 1984, Hase 1985]. Among these are investigations of overtone-induced unimolecular dissociation (for an overview, see Uzer and Hynes [1987]), and isomerization reactions [Hutchinson 1989]; i.e., processes in which the reaction energy is supplied by exciting the overtones of a $\mathrm{OH}$ or $\mathrm{CH}$ local mode (for overviews of the experimental situation in isomerization, see Jasinski et al. [1983, 1984], Segall and Zare [1988]; and in bond fission, Crim [1987]) or NH modes [Foy et al. 1989] (for recent theoretical work, see Hase [1985], Holme and Hutchinson [1986], Hutchinson [1989], Marshall and Hutchinson [1987], Hutchinson and Marshall [1988]; for general treatments of the dynamics of localized states see, e.g., Moiseyev [1983], Davis and Heller [1984]).

Since the reaction energy reaches the reaction coordinate by vibrational energy redistribution, these processes form an interface between IVR and reaction dynamics. The role of nonlinear resonances in 
model isomerization reactions were investigated by Uzer and Hynes [1985], and research on the isomerization of hydrogen cyanide [Smith et al. 1987] has assigned a role to combination resonances in changing the rate of reaction [Holme and Hutchinson 1985]. The energy transfer processes leading to isomerization in HONO have been examined by Guan et al. [1987]. Other studies of the interface between isomerization and IVR are Sumpter and Thompson [1987c], Hutchinson [1987], Spears et al. [1988a,b], R.P. Muller et al. [1988], Raff [1989].

The overtone-induced dissociation of hydrogen peroxide has become a prototypical unimolecular fission reaction where many of the ideas concerning intramolecular vibrational relaxation, reactivity, and the interpretation of linewidths have tended to come together. Calculations of the energy transfer leading to reaction [Sumpter and Thompson 1985, Uzer et al. 1985a, 1986, Sumpter and Thompson 1987a, 1988, Uzer et al. 1988, Getino et al. 1989], have, using different potential energy surfaces, tended to confirm a slow, sequential, two-step energy transfer mechanism [Uzer et al. 1985a, 1986]. The energy migrates from the $\mathrm{OH}$ stretching mode to the $\mathrm{OOH}$ wagging mode, which, in turn, channels it into the rest of the molecule, including the reactive $O O$ stretching motion. This transfer takes place on a timescale slow compared with molecular vibrations, and leaves the initially unexcited $\mathrm{OH}$ stretching mode as an inactive spectator because its high frequency precludes effective resonances with the rest of the molecule. While the detailed results are sensitive to the choice of force field [Getino et al. 1989], the robustness of the energy transfer mechanism suggests that hydrogen peroxide has some potential for mode-specific dissociation [Scherer et al. 1986, Scherer and Zewail 1987, Uzer et al. 1988]. Indeed, Chuang et al. [1983] and Gutow et al. [1988] report mode-specificity in the overtone-induced dissociation of the related system, t-butyl hydroperoxide. Dynamical experiments which, instead of deducing lifetimes indirectly from linewidths [Rizzo et al. 1984, Ticich et al. 1986, Butler et al. 1986b, Luo et al. 1988], use real-time investigations of the dissociation process [Scherer et al. 1986, Scherer and Zewail 1987] are expected to help resolve such issues. Among reactions, predissociation, especially in Van der Waals molecules [Miller 1986], is a process where the role of IVR has been probed extensively (e.g., Gray et al. [1986a], Ewing [1987], Ewing et al. [1987], Jucks and Miller [1987], Evard et al. [1988], Wozny and Gray [1988]). For the intricacies involved in connecting unimolecular decay rates from linewidths, the reader is referred to the appendix.

\subsection{Generalizing the pendulum}

It was mentioned earlier that during the reduction of the full nonlinear Hamiltonian into a pendulum [eqs. (5.9), (5.10)], it was necessary to approximate the Fourier coefficients by constants - by, for example, evaluating them at the resonance center. This approximation is only good when the coupling between the degrees of freedom is weak. For strong couplings, the variation of the Fourier coefficients with the momentum needs to be taken into account. An example of this procedure is provided by the Hamiltonian

$$
H_{\mathrm{V}}=\frac{1}{2} \omega\left(p_{1}^{2}+q_{1}^{2}+p_{2}^{2}+q_{2}^{2}\right)+k\left(q_{1}^{4}+q_{2}^{4}\right)+k_{12}\left(q_{1} q_{2}\right)^{2} .
$$

A single-resonance approximation to (5.17), when expressed in the action-angle variables of the unperturbed Hamiltonian, reads [Sahm and Uzer 1989a; Sahm et al. 1989]

$$
H=\frac{1}{4}\left(3 k-k_{12}\right) \Delta^{2}+\frac{1}{8} k_{12}\left(I^{2}-\Delta^{2}\right) \cos 4 \phi_{\Delta} .
$$

This Hamiltonian is similar to that of an asymmetric rotor in torque-free space. The relation between this and the pendulum Hamiltonian can be made more transparent by transforming the rotor 
Hamiltonian into conjugate variables $(K, \chi)$, where $K$ is the $z$-component of the angular momentum on the body-fixed axes. This transformation [Deprit 1967; Augustin and Miller 1974; Augustin and Rabitz 1979; Ezra 1986],

$$
J_{x}=-\left(J^{2}-K^{2}\right)^{1 / 2} \sin \chi, \quad J_{y}=-\left(J^{2}-K^{2}\right)^{1 / 2} \cos \chi, \quad J_{z}=K,
$$

converts the asymmetric top Hamiltonian

$$
H_{\mathrm{AR}}=C J_{x}^{2}+B J_{y}^{2}+A J_{z}^{2}
$$

into

$$
H_{\mathrm{AR}}=\frac{1}{2}(B+C) J^{2}+[A-(B+C) / 2] K^{2}+\frac{1}{2}(B-C)\left(J^{2}-K^{2}\right) \cos 2 \chi,
$$

which approximates the pendulum Hamiltonian (5.10) for small variations of $K$.

The replacement of the pendulum by an asymmetric top has been applied to vibrational energy transfer due to rotations, or Coriolis-induced vibrational mixing [Clodius and Shirts 1984; Uzer et al. 1985a; Sahm and Uzer 1989a; Sahm et al. 1989]. The transformations, when applied to the model vibration-rotation Hamiltonian

$$
H=H_{\mathrm{V}}+B \pi_{z}^{2}-2 B L \pi_{z}, \quad \pi_{z}=\zeta\left(p_{2} q_{1}-p_{1} q_{2}\right)
$$

with $B, \zeta, L$ constants, show that the energy-transfer picture between the two modes can be determined by considering the intersection of two solid figures

$$
H=C J_{x}^{2}+B J_{y}^{2}+A J_{z}^{2}+c J_{x}, \quad J^{2}=J_{x}^{2}+J_{y}^{2}+J_{z}^{2},
$$

in the space spanned by the vibrational variables $\left(J_{x}, J_{y}, J_{z}\right)$ [Sahm et al. 1989].

The semiaxes of the ellipsoid (5.23a), which is a "constant energy surface" [Harter and Patterson 1984; Harter 1984, 1988], are determined by the vibrational constants, and the radius of the sphere by the initial excitations. The angular momentum of the molecule determines the distance between the centers of the two solids. The same picture has also been applied to vibration-induced rotation mixing ("K-mixing") by Sahm and Uzer [1989b].

The basis for these developments is the homomorphism between the $\mathrm{SU}(2)$ and $\mathrm{R}(3)$ symmetry groups [Harter 1986]; these have some generality for resonances other than $1: 1$ because an arbitrary $m: n$ resonance can be transformed into a $1: 1$ resonance, i.e., can be made to show $\mathrm{SU}(2)$ symmetry [Farrelly 1986a]. Group-theoretical ideas have been very fruitful in understanding and classifying interactions between vibrations [Kellman 1982, 1985, 1986; Lehmann 1983, Benjamin and Levine 1983; Van Roosmalen et al. 1983, 1984; Levine and Kinsey 1986; Kellman and Lynch 1986a,b; 1988a,b; Jaffé 1988; Xiao and Kellman 1989].

\subsection{Nonstatistical effects and phase space structures}

In the introduction, we mentioned the two key assumptions of RRKM theory: Intramolecular energy relaxation is complete and fast compared with the time scale of unimolecular dissociation (statistical theories have been reviewed by Truhlar et al. [1983], Hase [1983], as well as Wardlaw and Marcus 
[1987]). Notwithstanding the widespread successes of the RRKM theory, even early on a number of experimental hypotheses did not seem to apply; among these "nonrandom decay" cases were some unimolecular reactions involving chemical activation [Forst 1973]. When statistical theory is applied to such reactions without any modifications, the results are at variance with the experiment. A particular severe case is the predissociation of Van der Waals molecules like $\mathrm{HeI}_{2}$, where a molecule with more than enough energy to dissociate lives for a very long time because the dissociation energy is in the "wrong" mode and tends to stay there (see Wozny and Gray [1988] for references).

Together with the realization that energy relaxation might sometimes be incomplete came empirical ways of accounting for such nonstatistical effects in reaction rate calculations. Corrections for nonstatistical relaxation include the exclusion of certain modes from rate calculations, thereby practically treating the molecule as a smaller molecule. For example, a vibrational mode may have much higher frequency than others and cannot couple with them strongly, and is therefore inactive in the energy transfer process [Davis 1985]. A recent example involves reaction rate calculations for hydrogen peroxide [Brouwer et al. 1987]. For other empirical procedures for correcting for slow relaxation, see for example, Marcus et al. [1984].

With the application of classical mechanics to the study of molecular systems (explained in the classical trajectories section) came the opportunity to observe, in microscopic detail, the dynamical processes that are assumed in the statistical theories. An immediate outcome of these studies has been the recognition that phase space is very complex; for realistic systems, it is not homogeneous, but full of structures. Moreover, all of these structures influence the motion of phase points in different ways. It is worth remembering at this point that the inclusion of quantum mechanics alters these phase space structures and their effect on dynamics. For more on the quantum-mechanical structure of phase space, see, for instance, Davis and Heller [1981], Heller and Davis [1981], Heller [1983], DeLeon et al. [1984], Davis and Heller [1984], Davis [1988], and Skodje et al. [1989]. Rather than delving into the impact of modern nonlinear dynamics on the study of molecular systems, let us start with an observation: when the time dependence of mode energies was studied (say in a model of OCS-more of that later), it was found that some systems were riddled with long-time correlations. Mode energies did not decay (or increase) monotonically to their statistical limit, but instead, phase points sometimes appeared to be trapped in certain regions of phase space for many vibrational periods. In other words, these systems failed to relax statistically. Clearly, one can expect the dissociation behavior of such molecular systems to be nonstatistical and nonrandom, also.

It had been known that at high energies, phase space may be divided such that quasiperiodic and chaotic regions coexist [Chirikov 1983]. While quasiperiodic components in the available phase space imply incomplete relaxation, long-time correlations lead to slow relaxation. For applying statistical theories, such "nonrandom" components must be discounted. Thus, DeLeon and Berne [1981] corrected their RRKM calculation for quasiperiodic motion by excluding it from their measure of phase space, much in the spirit of empirical corrections to statistical theories.

The advent of nonlinear dynamics has provided the needed support for a microscopic examination of phase space in general, and the problem of long-time correlations in particular [Contopoulos 1971, Chirikov 1979, DeLeon and Berne 1982a,b; Vivaldi et al. 1983; Meiss et al. 1983; Karney 1983; Casartelli 1983, Kato 1985a]. Some long-time correlations have been interpreted as "vague tori" [Reinhardt and Jaffé 1981; Jaffé and Reinhardt 1982; Shirts and Reinhardt 1982; Reinhardt 1982; Kuzmin et al. 1986a]. In the background to the theory, we discover the common thread that runs through this review, namely the influence of nonlinear resonances. For our purposes, findings on two-degree of freedom systems will be sufficient for making our point (for a discussion of systems with more degrees of freedom, see Lichtenberg and Lieberman [1983], Martens et al. [1987]). 
In the discussion of an isolated resonance, we have seen how resonance zones are bounded by separatrices. Irregular motion appears around separatrices at low energies. At higher energies, even as separatrices fragment, irregular motion may still fail to spread widely because it may be restricted to tori and KAM curves. The last KAM curve to disappear in the Standard Map is a torus that is formed when the ratio of (the two) local frequencies, $\alpha$, is related to the Golden Mean $\gamma$, viz.

$$
\alpha=1+\gamma, \quad \gamma=\left(5^{1 / 2}-1\right) / 2 \text {. }
$$

The break-up of this "Golden Torus" (which is a "cantorus" in the terminology of Percival [1979]) has attracted the attention of researchers searching for a transition to a global stochasticity [Greene 1968; Berry 1978; Greene 1979; Escande and Doveil 1981; Shenker and Kadanoff 1982; MacKay 1983]. Crudely speaking, the Golden Mean is the irrational number which is hardest to approximate by rationals [Berry 1978]. Intuitively, the "Golden Torus" as the last obstacle to unrestricted transport in phase space is appealing, because it is found in phase space regions where the coupled oscillators have the greatest difficulty of driving each other resonantly [Davis 1985].

Based on the cited earlier work on transition to global stochasticity, MacKay et al. [1984] and Bensimon and Kadanoff [1984] developed a quantitative approach to transport in area-preserving maps that gives rates of relaxation for systems of two degrees of freedom, even when long-time correlations, which are such stumbling blocks to traditional statistical relaxation approaches, exist [Meiss and Ott 1986]. While research into the fundamentals of diffusion is outside the scope of this review, the interested reader can consult Lichtenberg and Lieberman [1988], Lichtenberg and Wood [1989a,b] for coverage of some recent research. The application of these methods to intramolecular energy transfer was undertaken by Davis [1984] who used the term "intramolecular bottleneck" to describe phase space structures such as cantori since even the broken remnants of a KAM torus act as bottlenecks to the flow of phase points. This terminology emphasizes the connection of this recent work to earlier statistical theories; indeed, the work of MacKay et al. [1984] and Bensimon and Kadanoff [1984], possesses many of the features of statistical reaction rate theories. It is also reminiscent of periodic-orbit approach to chemical reactions [Pollak et al. 1980; Pollak 1985].

Using this work on phase-space transport, Davis [1985] modeled energy relaxation in highly excited OCS restricted to collinear geometry [Carter and Brumer 1982, 1983; Hamilton et al. 1982], where long-time correlations were previously observed [Davis and Wagner 1984; Davis 1984]. Figures 9a and b illustrate a Poincaré surface of section for a single trajectory. The surface of section is for the normal mode of OCS which is principally CS stretch in character, about $80 \%$ of CS character in the low-energy limit. In addition to the trajectory, which appears as dots, these figures show three curves. The outermost curve illustrates the energy boundary. The middle curve shows a dividing surface which is an approximation to the last KAM curve separating the two primary resonance zones. The innermost curve shows the boundary of a quasiperiodic region. Davis [1984] was able to calculate the flux through the dividing surface and calculate the relaxation rate for systems with such long-time correlations and slow randomization. A comparison of the quantum-mechanical intramolecular dynamics with the classical one for OCS has been performed by Gibson et al. [1987]. These calculations have been extended and the quantum-mechanical nature of states around these phase-space structures, especially in the chaotic regime, have been discussed by Davis [1988]. Although outside the scope of this overview, we mention that the role of phase-space bottlenecks in unimolecular reactions has been examined by Davis and Gray [1986], Gray et al. [1986b] as well as Gray and Rice [1987] and Tersigni and Rice [1988]. There are similar studies on bimolecular reactions by Davis [1987], Skodje and Davis [1988], and Skodje [1989]. The quantum-mechanical manifestations of these barriers have recently been 

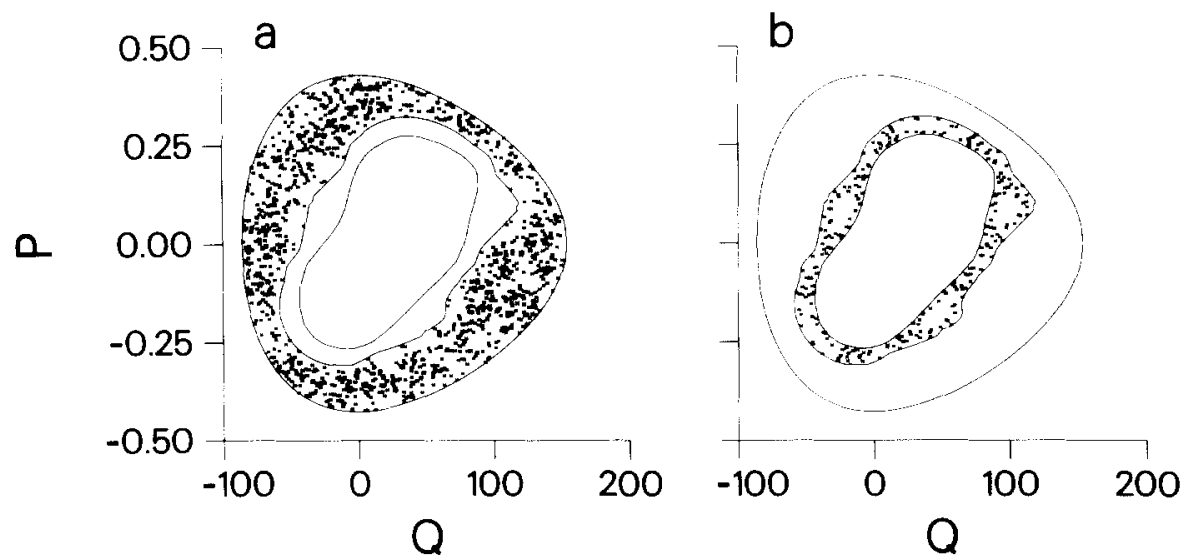

Fig. 9. The effect of a strong bottleneck is shown for collinear OCS at $E=20000 \mathrm{~cm}^{-1}$. a. For the first $24.6 \mathrm{ps}$ of propagation the trajectory moves outside of the dividing surface. $b$. For the next $4.5 \mathrm{ps}$ the trajectory is inside the dividing surface, but cannot enter the inner quasiperiodic region. (From Davis [1985])

uncovered by Brown and Wyatt [1986a,b], Geisel et al. [1986], and this work has been surveyed by Radons et al. [1989].

\section{6. "Mesoscopic" description of intramolecular vibrational energy transfer}

In the preceding four sections, we have seen the various determinants of intramolecular energy transfer. We started with a quantum-mechanical description, and studied energy transfer in a state-bystate fashion with few assumptions about the detailed nature of the couplings. Later, we went on to nonlinear oscillator chains and saw the importance of nonlinear resonances as well as the relevance of the KAM theorem for these scenarios. Finally, we described energy transfer through a single as well as overlapping resonances in great detail by means of classical mechanics. In this section we present a "mesoscopic" combination of the quantum statistical-mechanical view with the detail of the nonlineardynamical point of view [Kuzmin and Stuchebryukhov 1985; Kuzmin et al. 1986a; Stuchebrukhov et al. 1986; Stuchebryukhov 1986], which uses quantal Green function methods (see e.g., Couley [1963]) to calculate quantities and trends of interest for IVR when the coupling is explicitly known. The approach reported here is in contrast with the work of Hanson et al. [1985] and Meiss and Ott [1986], on Markov chains and trees in area-preserving maps where a (discrete-time) master equation is derived dynamically.

It will emerge, again explicitly, that under suitable conditions, there can be energy regions where a given excited mode fails to relax (for general references, see Kuzmin et al. [1987a,b]). Obviously, when modes in a highly excited molecule are decoupled in this manner above the dissociation energy, the dissociation of the molecule will not be governed by the ordinary statistical reaction rate theories. Such highly excited systems have been discussed by Noid and Koszykowski [1980], Wolf and Hase [1980b], Waite and Miller [1980, 1981, 1982], Hedges and Reinhardt [1983], Bai et al. [1983], Hose and Taylor [1984], Christoffel and Bowman [1982, 1983], Chuljian et al. [1984], Moiseyev and Bar-Adon [1984], Skodje et al. [1984a,b], Swamy et al. [1986], Chang et al. [1986], and Kuzmin et al. [1986b]. The work in this section has recently been reviewed, and its implication to molecular lineshapes and widths emphasized [Stuchebrukhov et al. 1989]. 


\subsection{Self-consistent description of quasiharmonic mode relaxation}

In a molecule, the frequencies of various modes are energy dependent, as are the relaxation rates of the excited modes. At high excitation levels, the molecule can be described in the self-consistent quasiharmonic approximation [Kuzmin and Stuchebrukhov 1985; Stuchebrukhov 1988] as an ensemble of vibrational quasimodes $\{k\}$ with energy-dependent frequencies $\omega_{k}(E)$. While the mode frequencies vary, each vibrational mode is modeled by a series of equally spaced levels (a brief justification will be given in the next section). Moreover, each mode has a corresponding (exponential) relaxation rate $\gamma_{k}(E)$. At low energies, the frequencies turn into normal-mode frequencies, and the $\gamma_{k}(E)=0$. As the energy of the molecule is raised above a certain critical value of $E_{\mathrm{c}}$, each mode begins to undergo damping at its own rate $\gamma_{k}$, which describes the energy exchange process among the modes. This threshold behavior of transition to a quasidissipative regime has been observed experimentally (e.g., Bagratashvili et al. [1981], Felker and Zewail [1984]) and in numerical modeling [Tennyson and Farantos 1984; Livi et al. 1985]. In this model, the appearance of nonzero $\gamma$ corresponds to a transition to chaotic intramolecular dynamics with exponential damping of the correlations [Koszykowski et al. 1981; Berman and Kolovsky 1983; Zaslavsky 1985].

As an example, consider the Hamiltonian

$$
H=\frac{1}{2} \sum_{i=1}^{S} \omega_{0 i}\left(p_{i}^{2}+q_{i}^{2}\right)+\frac{1}{3 !} \sum_{i, j, k=1}^{s} X_{i j k} q_{i} q_{j} q_{k},
$$

where $q_{i}$ are the normal coordinates and $p_{i}$ are the corresponding momenta. Assume that the nonlinearities $\{X\}$ are much smaller than the unperturbed frequencies, i.e., that this is a "degenerate" system very much like the one discussed by Ford and Lunsford [1970]; if the unperturbed frequencies $\omega_{0 i}$ satisfy certain resonance relations, randomization can occur even at very small molecular energies. But for large and intermediate size molecules, a more typical situation is one where the various resonances does not satisfy exact resonance relations, but approximate ones with characteristic detunings of the order 10-100 wavenumbers, and the randomization begins at finite energies $E_{\mathrm{c}}$. The appearance of relaxation in this model has a threshold character at finite energies, and moreover is in harmony with experimental and simulation studies, as discussed below (the qualitative change in the motion at a critical energy has been analyzed using critical point analysis in Cerjan and Reinhardt [1979]).

The existence of close resonances for several degrees of freedom leads to a situation where the critical energy $E_{\mathrm{c}}$ is much lower than the dissociation energy $D$, in contrast to much smaller systems like the Hénon-Heiles Hamiltonian (Oxtoby and Rice [1976]), where the resonances overlap with respect to one coordinate, and $E_{\mathrm{c}}$ is close to $D$ (for the inconclusive evidence concerning the existence of $E_{\mathrm{c}}$ for more than two degrees of freedom, see Lichtenberg and Lieberman [1983], chapter 6).

Information relevant to IVR, such as correlation functions, the absorption spectrum, and the relaxation characteristics, are contained in the system's linear response function, i.e., the retarded Green function [Couley 1963] which is used to compute the vibrational relaxation rate.

The calculation proceeds by the computation of the response of an ensemble of systems (6.1) to an infinitely weak external monochromatic field and following how this response changes as the energy of the molecule is increased. Molecular collisions are ignored and the only role that the ensemble plays is to ensure the appropriate averaging. The linear response of the system is determined by the correlation properties of the unperturbed motion; therefore, we are actually studying the motion of the isolated system (6.1) with averaged initial data. To simulate experimental conditions the calculation was performed for a thermal distribution. 
The response of the ensemble with the Hamiltonian (6.1) to an external perturbation is given by the retarded Green function

$$
G_{i k}^{\mathrm{R}}=\mathrm{i} \theta(t)\left\langle\left[q_{i}(t), q_{k}(0)\right]\right\rangle,
$$

where the $q_{i}(t)$ are the operators in the Heisenberg representation, $\theta(t)$ is the Heaviside step function, and the averaging is over the thermal distribution with the exact Hamiltonian (6.1)

$$
\langle\cdots\rangle=\operatorname{Tr}\left(\mathrm{e}^{-\beta H} \cdots\right) / \operatorname{Tr} \mathrm{e}^{-\beta H}
$$

The imaginary part of the Fourier transform $G^{\mathrm{R}}(\omega)$ is proportional to the absorption spectrum of the system. Consider the form of $G^{\mathrm{R}}(\omega)$ for a few simple cases. For a molecule in the harmonic approximation, i.e., when $X_{i j k}=0$, the frequency-dependent Green function is

$$
G_{i k}^{\mathrm{R}(0)}(\omega)=\frac{1}{2} \delta_{i k}\left[1 /\left(\omega+\omega_{0 k}\right)-1 /\left(\omega-\omega_{0 k}\right)\right] .
$$

The absorption spectrum consists of a single line at the frequency $\omega_{0 k}$, (the same frequency with the opposite sign gives the stimulated emission spectrum). If each of the harmonic modes were exponentially attenuated at the rate $\gamma_{k}$, as a result of, for example, contact with an infinite energy reservoir, then we have

$$
G_{i k}^{\mathrm{R}(0)}(\omega)=\frac{1}{2} \delta_{i k}\left(\frac{1}{\omega+\omega_{k}+\mathrm{i} \gamma_{k} / 2}-\frac{1}{\omega-\omega_{k}^{-+} \mathrm{i} \gamma_{k} / 2}\right) .
$$

The relaxation of energy in each of the modes to the equilibrium state occurs at a rate $\gamma_{k}$. In the case (6.5), the contribution of the $k$ th mode to the total absorption is given by the Lorentzian

$$
\sigma_{k}\left(\omega_{L}\right) \simeq \frac{1}{\pi} \frac{\gamma_{k} / 2}{\left(\omega_{L}-\omega_{k}\right)^{2}+\left(\gamma_{k} / 2\right)^{2}} .
$$

The details of the Green function calculation are given in the articles of Kuzmin et al. [1986a] and Stuchebryukhov [1986]. The exact expression for the $G_{i k}^{\mathrm{R}}$ contains a very large number of close lines lying in the absorption spectrum about $(\hbar \rho)^{-1}$ apart, but the low-lying lines in the absorption spectrum of the system, or the envelope of the spectrum can be obtained readily. The result is a set of nonlinear equations for the quasimode frequencies $\omega_{k}$ and the relaxation rates $\gamma_{k}$, viz.

$$
\begin{aligned}
\omega_{k}= & \omega_{0 k}-\frac{1}{16} \sum_{i j=1}^{S} X_{i j k}^{2}\left(\frac{\underline{\left(n_{i}+n_{j}+1\right)}\left(\omega_{i}+\underline{\omega}_{j}-\omega_{k}\right)}{\left(\omega_{i}+\omega_{j}-\omega_{k}\right)^{2}+\left(\gamma_{i}+\gamma_{j}+\gamma_{k}\right)^{2} / 4}+\frac{\left(n_{i}+n_{j}+1\right)\left(\omega_{i}+\omega_{j}+\omega_{k}\right)}{\left(\omega_{i}+\omega_{j}+\omega_{k}\right)^{2}+\left(\gamma_{i}+\gamma_{j}+\gamma_{k}\right)^{2} / 4}\right. \\
& +\frac{\left(n_{j}-n_{i}\right)\left(\omega_{i}-\omega_{j}-\omega_{k}\right)}{\left(\omega_{i}-\overline{\left.\omega_{j}-\omega_{k}\right)^{2}+\left(\gamma_{i}+\gamma_{j}+\gamma_{k}\right)^{2} / 4}+\frac{\left(n_{i}-n_{j}\right)\left(\omega_{i}-\omega_{j}-\omega_{k}\right)}{\left(\omega_{i}-\omega_{j}-\omega_{k}\right)^{2}+\left(\gamma_{i}+\gamma_{j}+\gamma_{k}\right)^{2} / 4}\right),} \\
\gamma_{k}= & \frac{1}{16} \sum_{i j=1}^{S} X_{i j k}^{2}\left(\frac{\left(n_{i}+n_{j}+1\right)\left(\gamma_{i}+\gamma_{j}-\gamma_{k}\right)}{\left(\omega_{i}+\omega_{j}-\omega_{k}\right)^{2}+\left(\gamma_{i}+\gamma_{j}+\gamma_{k}\right)^{2} / 4}+\frac{\left(n_{i}+n_{j}+1\right)\left(\gamma_{i}+\gamma_{j}+\gamma_{k}\right)}{\left(\omega_{i}+\omega_{j}+\omega_{k}\right)^{2}+\left(\gamma_{i}+\gamma_{j}+\gamma_{k}\right)^{2} / 4}\right. \\
& +\frac{\left(n_{j}-n_{i}\right)\left(\gamma_{i}-\gamma_{j}-\gamma_{k}\right)}{\left(\omega_{i}-\overline{\left.\left.\omega_{j}-\omega_{k}\right)^{2}+\overline{\gamma_{i}}+\gamma_{j}+\gamma_{k}\right)^{2} / 4}+\frac{\left(n_{i}-n_{j}\right)\left(\gamma_{i}-\gamma_{j}-\gamma_{k}\right)}{\left(\omega_{i}-\omega_{j}-\omega_{k}\right)^{2}+\left(\gamma_{i}+\gamma_{j}+\gamma_{k}\right)^{2} / 4}\right),}
\end{aligned}
$$


which must be solved self-consistently at every internal energy. Here, $n_{i}$ are the Boltzmann populations of the $i$ th mode at a temperature $T$ (for experimental determination of vibrational temperature, see Weitz and Flynn [1981]). In computing these, the unrenormalized frequencies can be used because the frequency shifts are very small compared to the frequencies themselves. The dependence of $\omega_{k}$ and $\gamma_{k}$ arises from their dependence on $n_{i, k}$. Clearly, when the system is harmonic, then the only solutions to the system are the normal modes which do not decay. These solutions survive at high energies but there, there exists another solution with $\gamma$ different from 0 , which becomes the principal solution. For higher temperatures, a number of useful expressions can be derived from (6.8) indicating how the Fermi Golden Rule is reached with the density of three-frequency resonances [Bagratashvili et al. 1985]. The model becomes less valid near the transition energy $E_{\mathrm{c}}$, as is characteristic of other mean-field theories.

When these equations are applied [Kuzmin et al. 1986a] to small polyatomic molecules like $\mathrm{CFCl}_{2} \mathrm{Br}$, the results are found to be in agreement with experiment [Bagratashvili et al. 1981; Felker and Zewail 1984]. In general, the $\gamma$ show a nonmonotonic increase with total molecule energy because of the intricate way many nonlinear resonances act in concert to bring about relaxation. Since the frequencies of the modes are energy dependent, the corresponding resonance detunings can either increase or decrease as the molecule energy increases. Of course, when detunings increase, $\gamma$ will tend to decrease. In particular, occasionally $\gamma$ can vanish at $E>E_{\mathrm{c}}$ (see fig. 10). This implies that there are no resonances favorable in this molecule to drive relaxation. In the same figure, the detuning of the nearest resonances is shown. In order for gamma to be nonzero, at least two resonances must overlap. The intimate connection between relaxation and the overlap of resonances is shown in fig. 11, which shows the dependence of $\gamma_{j}$ for this molecule on the normal-mode frequency $\omega_{01}$. We can clearly see the resonance structure of the dependence of $\gamma_{1}\left(\omega_{01}\right)$ for small anharmonicity constants. For large anharmonicity

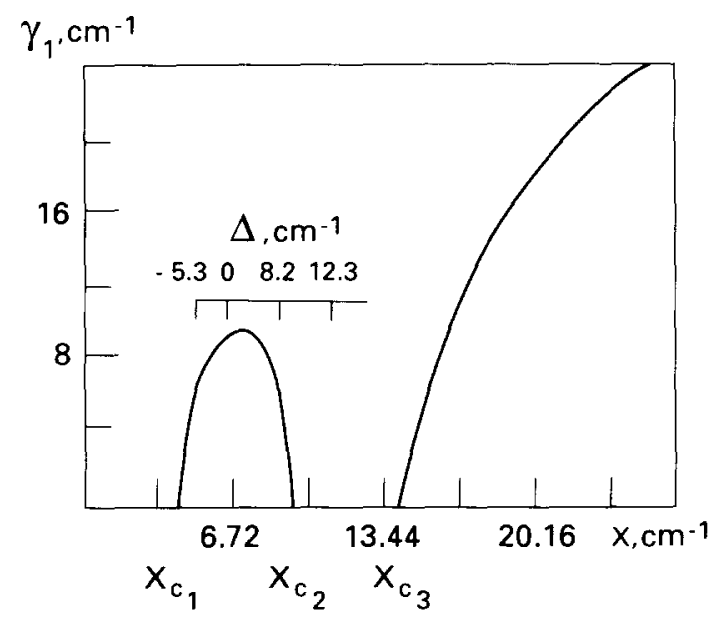

Fig. 10. Dependence of the intramolecular relaxation rate for mode 1 on the anharmonicity constant $X=X_{i j k}$. The system is one of nine oscillators with frequencies $1012,945,844,777,558,527,461,446$ and $380 \mathrm{~cm}^{-1}$. The energy of the system is constant, and equal to $4677 \mathrm{~cm}^{-1}$. The dependence of $\gamma(E)$ has a similar form: $\gamma=0$ in the regions $E<E_{\mathrm{c}}$ and $E_{\mathrm{c}_{2}}<E<E_{\mathrm{c}_{1}}$. (From Kuzmin et al. [1986a]).

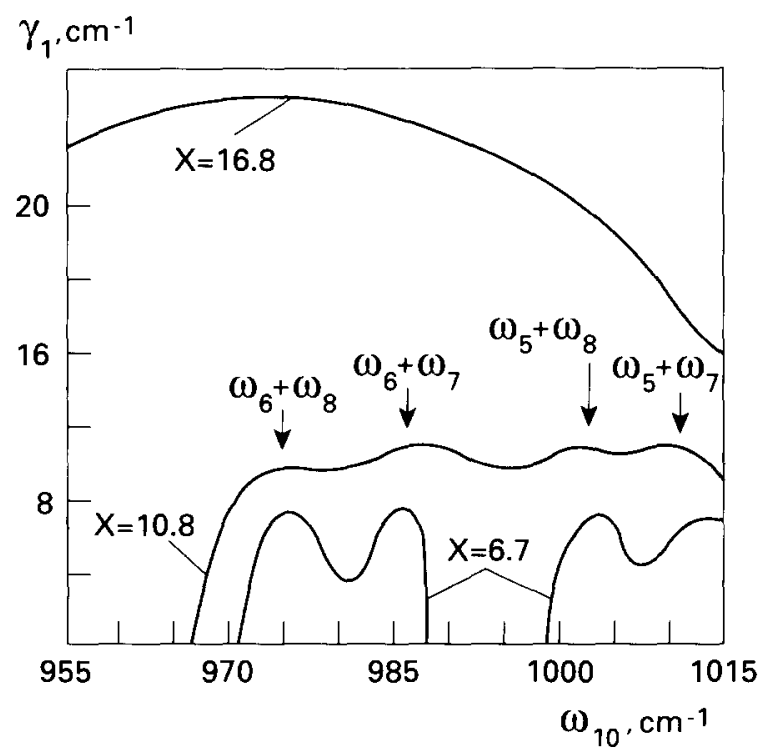

Fig. 11. Dependence of the intramolecular relaxation rate for mode 1 on the normal-mode frequency $\omega_{01}$. The system is the same as for fig. 10; the energy $E=4677 \mathrm{~cm}^{-1}$. (From Kuzmin et al. [1986a]). 


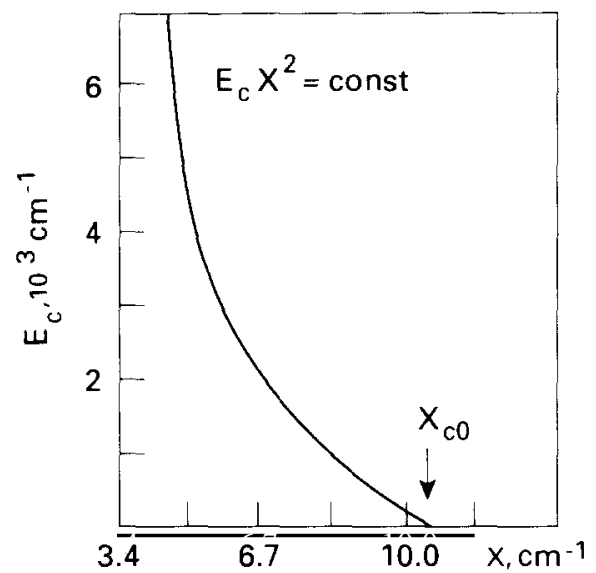

Fig. 12. Dependence of the critical energy $E_{\mathrm{c}}$ for the $\mathrm{CFCl}_{2} \mathrm{Br}$ molecule on the magnitude of the anharmonicity constant $X$. (From Kuzmin et al. [1986a]).

constants $X$, the effective interaction leads to the immediate overlapping of several resonances. Therefore the vanishing of $\gamma$ when $E>E_{\mathrm{c}}$ (fig. 10) can arise only in the case of moderate anharmonicity constants.

As to the dependence of the limit $E_{\mathrm{c}}$ on the system parameters, fig. 12 shows this dependence on the anharmonicity constant $X=X_{i j k}$. As expected, for very large anharmonicities, even zero-point vibrations are not independent of each other. The approximate dependence of $E_{\mathrm{c}}$ on $S$, the number of coupled degrees of freedom is, for three-frequency interactions, $E_{\mathrm{c}} \simeq\left(X^{2} S^{3}\right)^{-1}$, and this relation is accurate to $25 \%$ [Kuzmin et al. 1986a].

\subsection{Relaxation of individual quasiharmonic modes and its spectral signature}

We have seen in the preceding section that the relaxation of modes in a molecule can be described by a general set of nonlinear coupled algebraic equations, one set for the quasimode frequencies $\omega_{k}$, and another for the exponential relaxation rates $\gamma_{k}$.

In this section, we will focus on the relaxation characteristics of a single excited mode [Makri and Miller 1987], since many modern experiments (e.g., those involving overtone excitation, to name one class) approximate this scenario, and will examine the effect IVR has on the IR spectrum [Heller and Mukamel 1979; Kuzmin et al. 1987a,b; Stuchebrukhov and Khromov 1987; Ionov et al. 1988]. For the effect of collisional relaxation on spectral functions, see Koszykowski et al. [1982b]. Characteristic of the subsystem

$$
\gamma_{i}=\Gamma_{i}\left(\left\{\omega_{k}\right\},\left\{\gamma_{k}\right\}\right)
$$

is the fact that if the energy exceeds the threshold energy $E_{\mathrm{c}}$ greatly, the dependence of the right-hand sides $\Gamma_{i}$ on the variables $\left\{\gamma_{i}\right\}$ is very weak, and the correlation between the various solutions $\gamma_{i}$ disappears, and each of them can be found independently of the others by means of "Golden-Rule" type of recipes

$$
\gamma_{i}=2 \pi\left\langle V_{i}(E)\right\rangle^{2} \rho^{\mathrm{res}}\left(\omega_{i}\right)
$$


where $\left\langle V_{i}(E)\right\rangle$ is the effective interaction, which depends on the energy of the molecule, and $\rho^{\text {res }}$ is the density of the Fermi resonances. This implies that for such energies, we can in the treatment of molecular mode relaxation, treat the remaining modes as an energy reservoir, the correlation characteristics of which can be found independently of the state of mode $i$. In this section we will, along with Stuchebryukhov [1986] consider such a situation, and see that such an assumption enables us to investigate conditions in which purely phase relaxation can be separated from energy transfer processes.

The validity of this approach at high energies can be demonstrated [e.g., Zaslavsky 1985] by comparing relaxation times $\gamma_{i}^{-1}$ of the mode of interest, and the damping time $\tau_{c}$ of the correlations of the corresponding variables of the reservoir. For the separation of a selected mode and the dissipative reservoir, it is necessary that the former be much larger than the latter. (For high energies, this condition is valid for molecules, as shown in Bagratashvili et al. [1985], Stuchebryukhov [1986]).

The state of the molecule consisting of an excited mode (one which has been excited by a laser, say) and a reservoir of modes inactive in such absorption is specified by the density matrix $\boldsymbol{\sigma}$ of the excited mode and the vibrational temperature of the reservoir. This is also known as the "pumped mode" approach in multiphoton excitation (e.g., Mukamel [1978], Stephenson et al. [1979], Letokhov and Makarov [1981], Kay [1981], Stone et al. [1981], Lupo and Quack [1987]). For various theoretical descriptions of the multiphoton excitation processes, see Bagratashvili et al. [1985], Von Puttkamer et al. [1983], Lupo and Quack [1987], Gray et al. [1985], Rashev [1985], Rashev and Kancheva [1987], as well as references therein. The PM approach is not as practical as its alternative, many times referred to as the NME (nuclear molecular eigenstate) approach [Scheck and Jortner 1979] since it cannot determine the populations of excited levels directly. But within its framework it is possible to describe in detail the intramolecular relaxation processes (of the $T_{1}$ and $T_{2}$ kinds, e.g., Kay [1981], Stone et al. [1981], Budimir and Skinner [1987]). The procedure advocated by Bagratashvili et al. [1985] is different from a number of these multiphoton excitation (MPE) theories, because in addition to treating the vibrational temperature, the population in various vibrational levels and the spectroscopic anharmonicity constants, it also takes into account the relations between the vibrational frequencies, which turns out to be vital since it is the density of intermode resonances rather than the full level density that determines the efficiency of MPE. The multiphoton excitation dynamics in the laser field is described by a system of closed equations for $\boldsymbol{\sigma}$ and $T$ (these variables are coupled as a result of the possible exchange of energy between the excited mode and the reservoir).

On the other hand, the absorption spectrum, as well as the relaxation of the near equilibrium states can be studied at a fixed reservoir temperature. The interaction of the excited mode with the reservoir as a result of the anharmonic coupling terms gives rise to the processes of dephasing (e.g., Mukamel [1978]) and relaxation of the energy from the excited mode, and this is described by the corresponding relaxation operator $I^{\mathrm{R}}$ in the equation for $\boldsymbol{\sigma}$. Furthermore, the anharmonic interaction leads to a change in the dynamical part of the equation for $\boldsymbol{\sigma}$, a situation which can, in certain cases, be described as a redefinition of the Hamiltonian of the excited mode.

The simplest derivation of the kinetic equation is obtained when: (1) the Markovian approximation is valid, i.e., when the relaxation time $\tau$ of the system is much longer than the correlation time $\tau_{\mathrm{c}}$ for the reservoir; and (2) there is no frequency degeneracy in the system, i.e., if the pairs of levels $m, n$ and $m^{\prime}$, $n^{\prime}$, do not coincide, then

$$
\left|\omega_{m n}-\omega_{m^{\prime} n^{\prime}}\right| \gg \tau^{-1}
$$

where $\tau$ is the relaxation time of the system (this is often called the "secular approximation" in spin 
relaxation). The first condition is necessary for the kinetic equation to be differential rather than an integrodifferential equation, as in the case of a system with memory [Mukamel 1981]. The second condition leads to a situation in which the off-diagonal elements of the system's density matrix attenuate independently of each other, (the random-phase approximation) and the off-diagonal part of the kinetic equation has the form

$$
\mathrm{d} \sigma_{m n} / \mathrm{d} t=-\left(1 / T_{2}\right)_{m n} \sigma_{m n}
$$

where $T_{2}$ is the phase relaxation time, there being such a time for each pair of levels $m, n$. In the case when condition 2 is fulfilled, the off-diagonal elements relax like a set of uncoupled two-level subsystems. In the case that the system has frequency degeneracy, as in the case of the harmonic oscillator [Sazonov and Stuchebryukhov 1981, 1983; Parris and Silbey 1987], to take an extreme example, the relaxation of the off-diagonal elements occurs much more slowly. The various matrix elements are coupled and in the general case, the equation has the form

$$
\frac{\mathrm{d}}{\mathrm{d} t} \sigma_{m n}=\sum R_{m n, k l} \sigma_{k l}
$$

where the $R_{m n, k l}$ are coefficients. Thereby there is a distinctive coherence in the damping of the off-diagonal elements, and the system relaxes as a whole.

Since the relaxation of the off-diagonal elements determines the absorption spectrum of the system, the question of how this relaxation occurs is relevant for the study of the linear absorption spectrum (for sample computations of the spectrum, see Akulin and Karlov [1980], Makarov and Tyakht [1982]). It has been pointed out by Kay [1981] that allowance for the coherence effects discussed above can lead to a significant narrowing of the absorption spectrum. For further discussion of the effect of IVR on the spectrum, see True [1983].

Assume at first that the relaxation time of the system, $\tau$, is much larger than the correlation time $\tau_{\mathrm{c}}$. It is easily argued that condition 2 does not hold for an excited molecule. The quantity $\tau^{-1}$ determines the characteristic width of the absorption spectrum. This quantity is much larger than the anharmonic level shifts $\delta$, and it is indeed this condition that makes multiphoton excitation of polyatomic molecules possible in the quasicontinuum. The quantity $\delta$ also characterizes the remoteness of the levels of a vibrational mode, and lies in the range of 1 to 4 wavenumbers. The spectral width of the excited molecules ranges from values larger than 10-30 wavenumbers and the condition

$$
\tau^{-1} \gg \delta
$$

is fulfilled. But $\delta$ is also characteristic of the frequency difference $\left|\omega_{m n}-\omega_{m^{\prime} n^{\prime}}\right|$ and therefore the second condition above is not fulfilled. Indeed, the opposite is true, which indicates that the nonequidistance of levels is not important for the relaxation, and the vibrational mode can be modeled by a set of equally spaced levels. This is the quasiharmonic model [Bagratashvili et al. 1985] where the mode frequencies depend on the mode energy, but the levels remain equidistant.

In most past work it is implicitly assumed that condition 2 is fulfilled. This may lead to an overestimate of the width of the IR spectrum, which could be as large as an order of magnitude. In contrast to a number of articles where the above-discussed kinetic equation for the density matrix is used, the work of Kay [1981] is careful to take into account the second condition. It is also worth noting that condition 2 becomes even harder to satisfy in the limit $\hbar \rightarrow 0$ [Parson 1988]. 
Based on the preceding argument, many authors [Sazonov and Stuchebryukhov 1981, 1983; Bagratashvili et al. 1985; Kuzmin et al. 1986a; Stuchebrukhov 1988] have opted to use the quasiharmonic approximation rather than the random-phase approximation. The simplest variant of the kinetic equation with quasiharmonic modes was considered in Bagratashvili et al. [1985], where a special form for the nonlinear coupling was assumed, and purely phase relaxation was not considered.

Using the general method of Lax [1964], Stuchebryukhov [1986] derived kinetic equations describing the relaxation of selected vibrational modes of a molecule to the equilibrium state, and the role of various anharmonic terms causing relaxation are analyzed. They discuss the types of interactions that give rise to purely phase relaxation (by a similar, though not phenomenological process as Narducci et al. [1977]), and when their contribution to the shape of the absorption spectrum can be separated from the contributions of the energy relaxation process. Purely phase relaxation and its effect on the spectra of highly excited molecules has been considered recently by Makarov and Tyakht [1987], who, by using a formalism similar to that of Nitzan and Persson [1985] for adsorbates on surfaces (for other treatments, see Persson and Persson [1980], Van Smaalen and George [1987], Volotikin et al. [1986], Lin et al. [1988]), were able to suggest prescriptions for extracting dynamical information from the spectra of highly excited molecules, and to compare this relaxation with energy relaxation.

In the work of Stuchebryukhov [1986], it is shown that when allowance is made for high-order anharmonic interactions, the relaxation occurs nonexponentially (for other examples of nonexponential relaxation, see Dumont and Brumer $[1987,1988])$ and the absorption spectrum of a highly excited molecule is in general not Lorentzian (see, for example Bagratashvili et al. [1987]). Then the dependence of the longitudinal and transverse relaxation rates (in the case that they are separable) on the anharmonicity, the molecular frequencies, and the number of degrees of freedom can be investigated. They show that the effective molecular nonlinearity, which governs the relaxation process, depends on the energy of the molecule. Whereas at low energies the relaxation is governed by a three-frequency interaction, i.e., by nonlinearities of third order, at high energies the higher-order anharmonicities become activated and the relaxation proceeds by many-phonon processes. It is worth noting that at high energies, we can identify the nonlinearity order $m^{*}$ that makes the greatest contribution to the relaxation process. This order depends on the energy the molecule. At low energies it is three, and at energies close to the dissociation threshold, can attain the value of ten.

\section{Acknowledgement}

This article in its current form would have been impossible without the help and advice of many colleagues. I am grateful to J.M. Bowman, F. Budenholzer, M.J. Davis, D. Farrelly, M.R. Flannery, R.F. Fox, B.R. Foy, K.F. Freed, W.L. Hase, K.G. Kay, A.E.W. Knight, V. Lopez, R.A. Marcus, A. Nitzan, D.W. Noid, C.S. Parmenter, M. Quack, W.P. Reinhardt, D.W. Pratt, E.L. Sibert III, D.L. Thompson, G.A. Voth, and A. Zangwill for their contributions. Special thanks go to W.H. Miller for contributing the appendix, to R. Parson and A.A. Stuchebrukhov for many clarifications, as well as to E.W. McDaniel and Audrey D. Ralston, who were instrumental in the writing of this article. I also thank the United States National Science Foundation which supported me (through grant CHE 86-19298 to the Georgia Institute of Technology) during most of the writing of this article. I am grateful to the Atomic Collision Information Center at the Joint Institute for Laboratory Astrophysics, Boulder, Colorado for performing a literature search.

I dedicate this article to my colleague Joe Ford on the occasion of his 65 th birthday. 


\section{Appendix. On the relation between absorption linewidths, intramolecular vibrational energy redistribution (IVR), and unimolecular decay rates}

\section{W.H. Miller}

Here, a simple model (the standard one from the theory of radiationless transitions) is used to illustrate the relation (or lack thereof) between line widths in photoabsorption spectra and the unimolecular decay rate of the excited molecule. Among the relevant experiments are the recent photoabsorption spectra of ethylene dimers in a molecular beam. The point made is that a Lorentzianlike absorption line may indeed be unresolvable, even in principle, and still not be related to the lifetime of the molecule (i.e., the unimolecular decay rate).

With the current intense level of activity in high resolution vibrational spectroscopy and photodissociation of polyatomic molecules, there is considerable discussion as to the interpretation of line widths in the absorption spectrum with regard to rates of "intramolecular vibrational energy redistribution" (IVR) and, in the case of photodissociation, to unimolecular decay rates. The typical situation is that one observes a Lorentzian-like absorption line and wishes to interpret the width of the line as a rate; the question, which is addressed in this paper, is the rate of what? (The discussion throughout this paper relates to isolated molecule behavior; i.e., collisional and other environmental effects are assumed to be unimportant.)

The experiments that one has in mind are the excitation of overtones of $\mathrm{CH}$ stretch modes in polyatomic molecules [Henry 1977; Bray and Berry 1979; Reddy et al. 1982] and, more specifically, the photodissociation of Van der Waals clusters in supersonic molecular beams, [Smalley et al. 1976; Levy 1980; Cassassa et al. 1981; Hoffbauer et al. 1983; Fischer et al. 1983] though the same considerations arise in many other contexts. In the former case there is no dissociation involved, so there is no question of unimolecular decay; the essentially universal point of view [Heller and Mukamel 1979; Freed and Nitzan 1980; Stannard and Gelbart 1981; Sibert et al. 1982a,b] is that the $\sim 100 \mathrm{~cm}^{-1}$ width of the Lorentzian-like line gives the IVR rate of the initially excited local mode state. Under idealized, infinite resolution, of course, this Lorentzian-like "line" is actually a dense bundle of Dirac delta functions, the coefficients of which have a Lorentzian-like distribution, but this does not alter the IVR interpretation.

In the latter case, however-e.g., the photodissociation of ethylene dimers in a molecular beam [Cassassa et al. 1981; Hoffbauer et al. 1983, Fischer et al. 1983] - it is not immediately obvious what the $\sim 10 \mathrm{~cm}^{-1}$ width of the IR absorption line means. The Lorentzian-like line appears to be "homogeneous" by the most rigorous standards; i.e., unlike the case of $\mathrm{CH}$ overtone excitation, it appears that it would remain a single, smooth Lorentzian line even in the limit of infinite resolution. Assuming that this is indeed true, does the width of the line give the unimolecular decay rate (via the standard uncertainty relation)?

The purpose of this appendix is to illustrate the answer to this question by considering a simple model system. The model is borrowed from the standard theory of radiationless transitions, and indeed the absorption spectrum resulting from it is quite standard. The relation of this to the unimolecular decay rate is the somewhat nonstandard point that is the purpose of the appendix.

The model is the standard one in the theory of radiationless transitions [Avouris et al. 1977]: a zeroth-order state $|a\rangle$ is embedded in, and coupled to, a bath of zeroth-order states $|n\rangle$. It is state $|a\rangle$ that carries the oscillator strength from the initial, e.g., ground state of the molecule; i.e., there is no dipole matrix element from the ground state $|\mathrm{g}\rangle$ to any bath state $|n\rangle$. The bath states $|n\rangle$ are 
characterized by complex energies, $E_{n}-\mathrm{i} \Gamma_{n} / 2, E_{n}$ being their energy and $\Gamma_{n}$ their width for unimolecular decay (rate $=\Gamma_{n} / \hbar$ ). The optically allowed state $|\mathrm{a}\rangle$ is assumed to be nondissociative, i.e., characterized by the real energy $E_{\mathrm{a}}$.

The molecular system is thus characterized by the complex Hamiltonian matrix [Heller et al. 1978]

$$
\left(\begin{array}{lll}
E_{\mathrm{a}}, & H_{\mathrm{a}, n} & \rightarrow \\
H_{n, \mathrm{a}}, & E_{n}-\mathrm{i} \Gamma_{n} / 2, & 0 \\
\downarrow & 0 & \searrow
\end{array}\right)
$$

where $H_{\mathrm{a}, n}$ is the matrix element coupling state $|\mathrm{a}\rangle$ to various bath states $|n\rangle$. The complex eigenvalues of this matrix give the energies and lifetimes of the eigen-metastable states of the isolated molecule.

The absorption spectrum of the system, initially in its ground state, is given by

$$
\begin{aligned}
& I(E)=-\pi^{-1} \operatorname{Im}\langle\mathrm{g}|\mu G(E) \mu| \mathrm{g}\rangle, \\
& G(E)=\lim _{\varepsilon \rightarrow 0}(E+\mathrm{i} \varepsilon-H)^{-1}, \quad E=\hbar \omega+E_{\mathrm{g}} .
\end{aligned}
$$

Because of the assumption that $\mu|\mathrm{g}\rangle$ only has components along state $|\mathrm{a}\rangle$, this becomes

$$
I(E)=|\langle\mathrm{a}|\mu| \mathrm{g}\rangle|^{2}\left(-\pi^{-1}\right) \operatorname{Im}\langle\mathrm{a}|G(E)| \mathrm{a}\rangle
$$

Also of interest is the survival probability of the initial state $|a\rangle$

$$
P_{\mathrm{a} \leftarrow-\mathrm{a}}(t) \equiv\left|\left\langle\mathrm{a}\left|\mathrm{e}^{-\mathrm{i} H t / \hbar}\right| \mathrm{a}\right\rangle\right|^{2},
$$

and the survival probability $P(t)$ of the molecule itself,

$$
\begin{aligned}
& P(t) \equiv P_{\mathrm{a} \leftarrow \mathrm{a}}(t)+\sum_{n} P_{n \leftarrow \mathrm{a}}(t), \\
& P_{n \leftarrow \mathrm{a}}(t)=\left|\left\langle n\left|\mathrm{e}^{-\mathrm{i} H t / \hbar}\right| \mathrm{a}\right\rangle\right|^{2} .
\end{aligned}
$$

The survival probability of state $|\mathrm{a}\rangle, P_{\mathrm{a} \leftarrow \mathrm{a}}(t)$, is the probability that at time $t$ the system is still in the initially excited state $|\mathrm{a}\rangle$, while the survival probability of the molecule, $P(t)$ is the probability that by the time $t$ the molecule has not decomposed. If the Hamiltonian matrix in eq. (A.1) were Hermitian (i.e., $\left.\Gamma_{n}=0\right)$, then the propagator $\mathrm{e}^{-\mathrm{i} H t / \hbar}$ would be unitary and the total probability $P(t)$ conserved. This is the situation usually considered for radiationless transitions, and for example applies to the $\mathrm{CH}$ overtone excitation discussed in the introduction: $P(t)=1$ for all $t$, but is still the case that $P_{\mathrm{a} \leftarrow \mathrm{a}}(t)$ decays.

Matrix elements of the propagator in eqs. (A.5), (A.7) are most easily obtained by Fourier transforming the Green's function,

$$
\mathrm{e}^{-\mathrm{i} H t / \hbar}=-(2 \pi \mathrm{i})^{-1} \int_{-\infty}^{\infty} \mathrm{d} E \mathrm{e}^{-\mathrm{i} E t / \hbar} G(E),
$$


so that

$$
\begin{aligned}
& P_{\mathrm{a} \leftarrow \mathrm{a}}(t)=\left|-(2 \pi \mathrm{i})^{-1} \int_{-\infty}^{\infty} \mathrm{d} E \mathrm{e}^{-\mathrm{i} E t / \hbar}\langle\mathrm{a}|G(E)| \mathrm{a}\rangle\right|^{2} \\
& P_{n \leftarrow \mathrm{a}}(t)=\left|-(2 \pi \mathrm{i})^{-1} \int_{-\infty}^{\infty} \mathrm{d} E \mathrm{e}^{-\mathrm{i} E t / \hbar}\langle n|G(E)| \mathrm{a}\rangle\right|^{2} .
\end{aligned}
$$

Therefore, to determine the absorption spectrum, eq. (A.4), and the survival probabilities [Eqs. (A.5)-(A.7)], it is necessary only to have the a-a and a- $n$ matrix elements of the Green's function. For the model Hamiltonian of eq. (A.1) one readily obtains

$$
\begin{aligned}
& \langle\mathrm{a}|G(E)| \mathrm{a}\rangle=\left(E-E_{\mathrm{a}}-\sum_{n} \frac{\left|H_{n, \mathrm{a}}\right|^{2}}{E-E_{n}+\mathrm{i} \Gamma_{n} / 2}\right)^{-1}, \\
& \langle n|G(E)| \mathrm{a}\rangle=H_{n, \mathrm{a}}\left(E-E_{n}+\mathrm{i} \Gamma_{n} / 2\right)^{-1}\langle\mathrm{a}|G(E)| \mathrm{a}\rangle .
\end{aligned}
$$

To proceed further it is necessary to have the energies $E_{\mathrm{a}},\left\{E_{n}\right\}$, the lifetimes $\left\{\Gamma_{n}\right\}$, and matrix elements $H_{n, \mathrm{a}}$, which would require a very detailed molecular model (at best $a b$ initio quantum chemistry calculations). For illustrative processes we consider here the simplified version of the model first used by Bixon and Jortner [1968]: the bath energies $\left\{E_{n}\right\}$ are assumed to be equally spaced and the coupling elements $H_{n, \text { a }}$ and decay widths $\Gamma_{n}$ constant,

$$
E_{n}=n \varepsilon, \quad n=0, \pm 1, \pm 2, \ldots ; \quad \Gamma_{n}=\Gamma_{\mathrm{D}} ; \quad H_{n, \mathrm{a}}=V
$$

The sum over bath states in eq. (A.10) can then be evaluated, and one obtains

$$
\begin{aligned}
& \langle\mathrm{a}|G(E)| \mathrm{a}\rangle=\left[E-E_{\mathrm{a}}-\frac{1}{2} \Gamma_{\mathrm{I}} \cot \left(\pi E / \varepsilon+\mathrm{i} \pi \Gamma_{\mathrm{D}} / 2 \varepsilon\right)\right]^{-1}, \\
& \langle n|G(E)| \mathrm{a}\rangle=\left(\varepsilon \Gamma_{\mathrm{I}} / 2 \pi\right)^{1 / 2}\left(E-n \varepsilon+\frac{1}{2} \mathrm{i} \Gamma_{\mathrm{D}}\right)^{-1}\langle\mathrm{a}|G(E)| \mathrm{a}\rangle, \\
& \Gamma_{\mathrm{I}}=2 \pi V^{2} / \varepsilon .
\end{aligned}
$$

The model is thus characterized by the unimolecular decay width $\Gamma_{\mathrm{D}}$ and the width (i.e., rate) for IVR, $\Gamma_{\mathrm{I}}$, which is seen to be the Golden Rule width for the decay of state $|\mathrm{a}\rangle$. For the remainder of the appendix energy is measured in units of the bath level spacing $\varepsilon-$ i.e., one sets $\varepsilon=1-$ and also $E_{\mathrm{a}}$ is set to zero. (Taking $E_{\mathrm{a}}$ different from zero has only minor effects that are of no concern for the present considerations.)

Utilizing eq. (A.12) (with $\varepsilon=1, E_{\mathrm{a}}=0$ ), eq. (A.4) for the absorption spectrum becomes

$$
\begin{aligned}
& I(E)=|\langle\mathrm{a}|\mu| \mathrm{g}\rangle|^{2} \pi^{-1}(\Gamma / 2) /\left[(E-\Delta)^{2}+(\Gamma / 2)^{2}\right], \\
& \Gamma=\Gamma_{1} \frac{T\left(1+t^{2}\right)}{T^{2}+t^{2}}, \quad \Delta=\frac{\Gamma_{1}}{2} \frac{t\left(1-T^{2}\right)}{T^{2}+t^{2}}
\end{aligned}
$$




$$
t=\tan (\pi E), \quad T=\tanh \left(\frac{1}{2} \pi \Gamma_{\mathrm{D}}\right) .
$$

Examples of the spectrum will be shown in the next section, but here it is useful to note that for $\Gamma_{\mathrm{D}} \gg 1$ (which in practice means that $\Gamma_{\mathrm{D}} \geqslant 2$ ) one has $T \rightarrow 1$, so that the quantities in eq. (A.15) become

$$
\Gamma \rightarrow \Gamma_{\mathrm{I}}, \quad \Delta \rightarrow 0
$$

i.e., when the unimolecular decay width $\Gamma_{\mathrm{D}}$ is larger than the level spacing, $I(E)$ becomes a simple Lorentzian whose width is the IVR width $\Gamma_{\mathrm{I}}$.

It is somewhat more difficult to determine the survival probabilities $P_{\mathrm{a} \leftarrow \mathrm{a}}(t)$ and $P(t)$ in simple

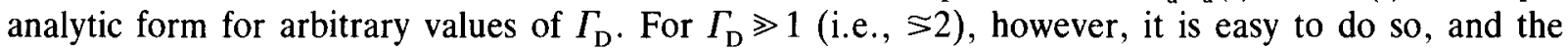
results are

$$
P_{\mathrm{a} \leftarrow \mathrm{a}}(t)=\mathrm{e}^{-\Gamma_{\mathrm{I}} t / \hbar}, \quad P(t)=\left(\Gamma_{\mathrm{I}} \mathrm{e}^{-\Gamma_{\mathrm{D}} t / \hbar}-\Gamma_{\mathrm{D}} \mathrm{e}^{-\Gamma_{\mathrm{I}} t / \hbar}\right) /\left(\Gamma_{\mathrm{I}}-\Gamma_{\mathrm{D}}\right)
$$

First, consider the absorption spectrum; this is very standard fare. Figures 13, 14 and 15 show $I(E)$, from eqs. (A.14), (A.15) for $\Gamma_{\mathrm{D}}=0.5,1$ and 2, respectively. $\Gamma_{\mathrm{I}}=10$ for all cases. Recall that the energy spacing of the bath, $\varepsilon$, is unity.

Figure 13 shows that one has a resolved spectrum which "sees" individual molecular eigenstates when the unimolecular decay width $\Gamma_{\mathrm{D}}$ is less than the energy level spacing of the bath. Figure 14 shows the intermediate case $\Gamma_{\mathrm{D}}=1$, and fig. 15 the case that $\Gamma_{\mathrm{D}}=2$ (twice the energy level spacing). If $\Gamma_{\mathrm{D}}$ is increased to any value $>2$, (even $\Gamma_{\mathrm{D}} \rightarrow \infty$ ), the spectrum $I(E)$ is imperceptibly changed from that in fig. 15.

Next consider the survival probabilities. From eq. (A.18a) one sees that in all cases the survival probability of state $|\mathrm{a}\rangle, P_{\mathrm{a} \rightarrow \mathrm{a}}(t)$ decays with the IVR rate $\Gamma_{\mathrm{I}} / \hbar$. The unimolecular decay rate that one infers from eq. (A.18b) is

$$
k_{\text {uni }}=\min \left(\Gamma_{\mathrm{D}}, \Gamma_{\mathrm{l}}\right) / \hbar
$$

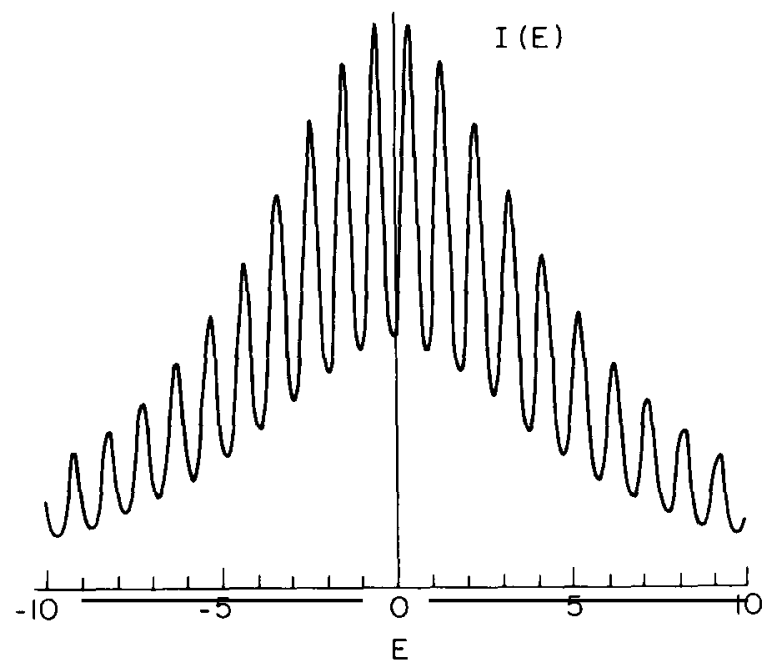

Fig. 13. Absorption spectrum (in arbitrary units) given by eqs. (A.14)-(A.16), for the case $\Gamma_{\mathrm{D}}=0.5 .\left(\Gamma_{\mathrm{I}}=10\right.$ for figs. 13-15, and the bath level spacing $\varepsilon=1$.) 


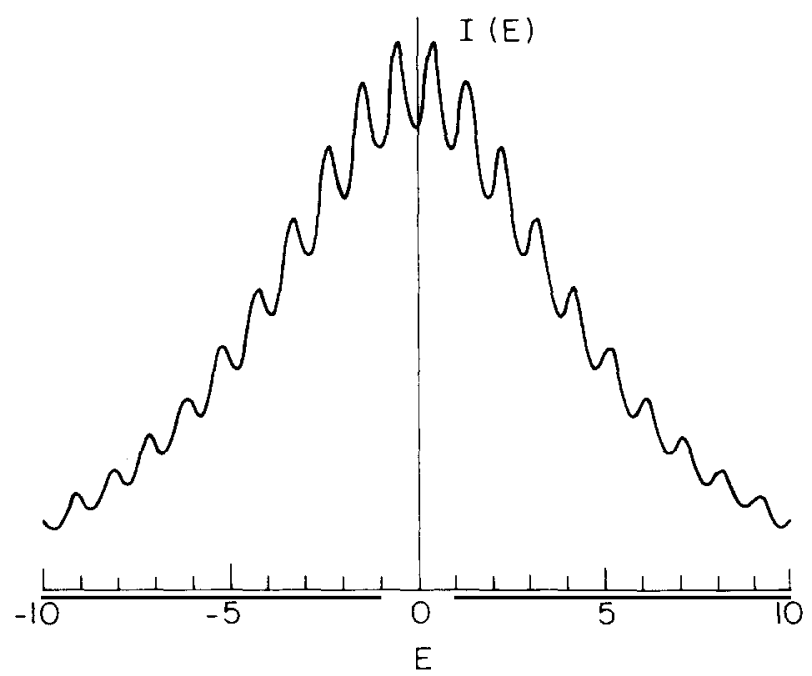

Fig. 14. Absorption spectrum (in arbitrary units) given by eqs. (A.14)-(A.16), for the case $\Gamma_{\mathrm{D}}=1 .\left(\Gamma_{1}=10\right.$ for figs. $13-15$, and the bath level spacing $\varepsilon=1$.)

For all the cases in figs. 13-15, therefore, the unimolecular decay rate is $\Gamma_{\mathrm{D}} / \hbar$, but if $\Gamma_{\mathrm{D}}>\Gamma_{\mathrm{I}}(=10$ here), then it is given by $\Gamma_{\mathrm{I}} / \hbar$. This has the obvious interpretation that for $\Gamma_{\mathrm{D}}<\Gamma_{\mathrm{I}}$, decay of state $|\mathrm{a}\rangle$ into the bath is fast (rate $=\Gamma_{\mathrm{I}} / \hbar$ ) and decay out of the bath slow (rate $=\Gamma_{\mathrm{D}} / \hbar$ ), while the converse is true for $\Gamma_{\mathrm{D}}>\Gamma_{\mathrm{I}}$. In either case the unimolecular decay rate is the rate limiting step, the smaller of $\Gamma_{\mathrm{I}} / \hbar$ and $\Gamma_{\mathrm{D}} / \hbar$. [For $\Gamma_{\mathrm{D}}=\Gamma_{\mathrm{I}} \equiv \Gamma$ eq. (A.18b) gives $P(t)=(1+\Gamma t / \hbar) \mathrm{e}^{-\Gamma t / \hbar}$, but still one has $\left.-\lim _{t \rightarrow \infty} \mathrm{d} \ln P(t) / \mathrm{d} t=\Gamma / \hbar.\right]$

The important observations from these results are:

(1) if $\Gamma_{\mathrm{D}}$ is less than the molecular energy level spacing, then the width of the individual lines does indeed give the unimolecular decay rate $\Gamma_{\mathrm{D}} / \hbar$;

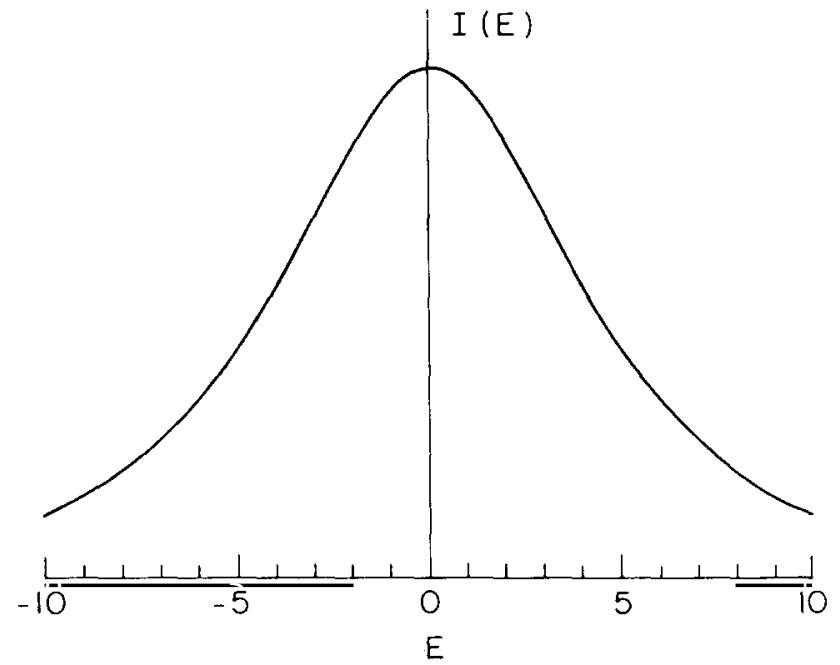

Fig. 15. Absorption spectrum (in arbitrary units) given by eqs. (A.14)-(A.16), for the case $\Gamma_{\mathrm{D}}=2 .\left(\Gamma_{1}=10\right.$ for figs. $13-15$, and the bath level spacing $\varepsilon=1$.) 
(2) however, if $\Gamma_{\mathrm{D}}$ is greater than the level spacing, there is no way to identify the unimolecular decay rate from the absorption spectrum; and

(3) in any event, $\Gamma_{\mathrm{D}}<1$ or $\Gamma_{\mathrm{D}}>1$ (i.e., figs. 13,14 or 15 ), the width of the complete band, resolved or unresolved, may be interpreted as the rate of IVR, $\Gamma_{\mathrm{I}}$, which is the rate of decay of the initially excited optically active state $|a\rangle$.

What, then, can one conclude in the case that a smooth Lorentzian-like line is observed, as for the photodissociation of ethylene dimer discussed in the introduction, which is clearly broader than the molecular energy level spacing (which can be estimated by statistical approximations)? First, one can clearly identify the observed width as $\Gamma_{\mathrm{I}}$, the width for IVR, which gives the rate for decay of the optically active zeroth order state initially excited. All that one can conclude about the unimolecular decay rate, however, is that it lies between $\varepsilon / \hbar$ ( $\varepsilon=$ molecular energy level spacing) and $\Gamma_{\mathrm{I}} / \hbar$. There is no way in principle, even with arbitrarily high resolution, to determine the unimolecular decay rate from the absorption spectrum. (It should be noted, though, that other excitation mechanisms may exist, involving operators other than the dipole operator, which excite individual eigenmetastable states, and in this case the linewidth would indeed give the unimolecular decay rate of the individual eigenmetastable states.)

\section{Acknowledgement}

This work has been supported by the National Science Foundation Grant CHE-79-20181.

\section{References}

Akulin, V.M. and N.V. Karlov, 1980, Redistribution of the vibrational energy in the course of laser excitation of high vibrational levels of polyatomic molecules, Sov. Phys. - JETP 79, 1063.

Alhassid, Y. and R.D. Levine, 1979, Collision experiments with partial resolution of final states: maximum entropy procedure and surprisal analysis, Phys. Rev. C 20, 1775.

Amirav, A. and J. Jortner, 1986, Rotational and vibrational state dependence on intramolecular coupling and dynamics in the $S_{1}$ state of pyrazine, J. Chem. Phys. 84, 1500.

Amirav, A. and J. Jortner, 1987, Rotation, vibration, and electronic relaxation, in: Stochasticity and Intramolecular Redistribution of Energy, eds R. Lefebvre and S. Mukamel (Reidel, Dordrecht) p. 171.

Apel, E.C. and E.K.C. Lee, 1986, Rotation-induced vibrational mixing in $S_{1} \mathrm{H}_{2} \mathrm{CO}$ near $E_{\mathrm{vib}}=2000-2300 \mathrm{~cm}^{-1}: 2^{1} 4^{3}$ and $2^{1} 4^{1} 6^{1}$ levels, J. Chem. Phys. 85, 1261.

Arnol'd, V.I., 1963a, Proof of a theorem of A.N. Kolmogorov on the invariance of quasiperiodic motions under small perturbations of the Hamiltonian, Russian Math. Surveys 18, 9.

Arnol'd, V.I., 1963b, Small denominators and problems of stability of motion in classical and celestial mechanics, Russian Math. Surveys $18,85$.

Augustin, S.D. and W.H. Miller, 1974, Semiclassical treatment of atom-asymmetric rotor collisions; rotational excitation of formaldehyde at low energies, J. Chem. Phys. 61, 3155 .

Augustin, S.D. and H. Rabitz, 1979, Action-angle variables in quantum mechanics, J. Chem. Phys. 71, 4956.

Avouris, P., W.M. Gelbart and M.A. El-Sayed, 1977, Nonradiative electronic relaxation under collision-free conditions. Chem. Rev. $77,793$.

Baggott, J.E., M.C. Chuang, R.N. Zare, H.-R. Dübal and M. Quack, 1985, Structure and dynamics of the excited $\mathrm{CH}_{-}$-chromophore in $\left(\mathrm{CF}_{3}\right)_{3} \mathrm{CH}_{\text {. }}$ J. Chem. Phys. 82, 1186.

Baggott, J.E., H.J. Clase and I.M. Mills, 1986, Overtone band shapes and IVR: C-H stretching overtones in $\mathrm{CHCl}_{3}, \mathrm{~J}$. Chem. Phys. 84, 4193.

Bagratashvili, V.N., Y.G. Vainer, V.S. Dolzhikov, S.F. Kol'yakov, V.S. Letokhov, A.A. Makarov, L.P. Malyavkin, E.A. Ryabov, E.C. Sil'kis and V.D. Titov, 1981, Intermolecular and intramolecular distribution of vibrational energy under multiphoton excitation by IR laser radiation. Sov. Phys. - JETP 53, 512.

Bagratashvili, V.N., M.V. Kuzmin, V.S. Letokhov and A.A. Stuchebrukhov, 1985, Theory of multiple-photon IR excitation of polyatomic molecules in the model of active and passive modes of a vibrational reservoir, Chem. Phys. 97, 13. 
Bagratashvili, V.N., S.I. Ionov, V.S. Letokhov, V.N. Lokhman, G.N. Makarov and A.A. Stuchebryukov, 1986, Uniform spectrum of vibrational transitions and the intramolecular vibrational relaxation time of a highly excited polyatomic molecule, JETP Lett. 44, 450.

Bagratashvili, V.N., V.N. Burimov, S.I. Ionov, A.P. Sviridov, A.A. Stuchebrukhov and I.M. Turovetz, 1987, Infrared spectroscopy and intramolecular vibrational relaxation of $\mathrm{c}_{-} \mathrm{C}_{6} \mathrm{~F}_{12}$ excited above the dissociation threshold, Chem. Phys. Lett. 137, 45

Bai, Y.Y., G. Hose, C.W. McCurdy and H.S. Taylor, 1983, Mode specificity of unimolecular rate constants in the Hénon-Heiles system, Chem. Phys. Lett. 99, 342.

Bain, A.J., P.J. McCarthy and R.M. Hochstrasser, 1986, Picosecond polarization spectroscopy as a probe on intramolecular dynamics: rovibronic relaxation in the $S_{1}$ state of trans-stilbene, Chem. Phys. Lett. 125, 307.

Benjamin, I. and R.D. Levine, 1983, Overtone spectrum in terms of normal or of equivalent modes with application to $\mathrm{H}_{2} \mathrm{O}$, Chem. Phys. Lett. $101,518$.

Bensimon, D. and L.P. Kadanoff, 1984, Extended chaos and the disappearance of KAM trajectories, Physica D $13,82$.

Berman, G.P. and A.R. Kolovsky, 1983, Correlation function behavior in quantum systems which are classically chaotic, Physica D 8, 117.

Berman, G.P. and G.M. Zaslavsky, 1977, Theory of quantum nonlinear resonance, Phys. Lett. A 61, 295.

Berman, G.P., G.M. Zaslavskii and A.R. Kolovskii, 1981, Interaction of quantum nonlinear resonance, Sov. Phys. - JETP 54, 272.

Berry, M.V., 1978, Regular and irregular motion, in: Topics in Nonlinear Dynamics, American Institute of Physics Conf. Proc. No. 46, ed. S. Jorna (AIP, New York) p. 16.

Bintz, K.L., D.L. Thompson and J.W. Brady, 1986a, Classical dynamics study of intramolecular energy flow in benzene, J. Chem. Phys. 85, 1848.

Bintz, K.L., D.L. Thompson and J.W. Brady, 1986b, Influence of anharmonicity of "bath" modes on intramolecular energy transfer from a local mode, Chem. Phys. Lett. 131, 398.

Bintz, K.L., D.L. Thompson and J.W. Brady, 1987, Intramolecular vibrational relaxation of CH stretch overtones in benzene, J. Chem. Phys. 86, 4411.

Birkhoff, G.D., 1927, Dynamical Systems (American Mathematical Society Colloquium Publications, New York) p. 82.

Bixon, M. and J. Jortner, 1968, Intramolecular radiationless transitions, J. Chem. Phys. 48, 715.

Bixon, M. and J. Jortner, 1969a, Long radiative lifetimes of small molecules, J. Chem. Phys. 50, 3284.

Bixon, M. and J. Jortner, 1969b, Electronic relaxation in large molecules, J. Chem. Phys. 50, 4061.

Bloembergen, N. and A.H. Zewail, 1984, Energy redistribution in isolated molecules and the question of mode-selective laser chemistry revisited, J. Phys. Chem. 88, 5459 .

Boeglin, A., A.A. Villaeys, R. Voltz and S.H. Lin. 1983, Dynamical effect of vibrational relaxation on electronic relaxation, J. Chem. Phys. 79, 3819.

Bogdanov, V.L., 1980, Subpicosecond relaxation and hot energy transfer accompanying excitation of the higher electronic state of organic molecules, J. Appl. Spectrosc. (USA) 33, 727

Bohr, N. and J.A. Wheeler, 1939, The mechanism of nuclear fission, Phys. Rev. 56, 426

Bondybey, V.E., 1984, Relaxation and vibrational energy redistribution processes in polyatomic molecules, Annu. Rev. Phys. Chem. $35,591$.

Born, M., 1960, The Mechanics of the Atom (Ungar, New York).

Bowman, J.M., 1985, Reduced dimensionality theories of quantum reactive scattering, Adv. Chem. Phys. 61, 115.

Bowman, J.M., 1986, The self-consistent field approach to polyatomic vibrations, Acct. Chem. Res. 19, 202.

Bowman, J.M., B. Gazdy and Q. Sun, 1989, A method to constrain vibrational energy in quasiclassical trajectory calculations, J. Chem. Phys. 91, 2859.

Brabham, D.E. and D.S. Perry, 1984, Local mode treatment of normal mode molecules: application to $\mathrm{CF}_{2} \mathrm{Cl}_{2}$ overtone spectra, Chem. Phys. Lett. 103,487

Bray, R.G. and M.J. Berry, 1979, Intramolecular rate processes in highly vibrationally excited benzene, J. Chem. Phys. 71, 4909.

Brouwer, L., C.J. Cobos, J. Troe, H.-R. Dubal and F.F. Crim, 1987, Specific rate constants $k(E, J)$ and product state distributions in simple bond fission reactions. II. Application to $\mathrm{HOOH} \rightarrow \mathrm{OH}+\mathrm{OH}, \mathrm{J}$. Chem. Phys. 86, 6171.

Brown, R.C. and R.E. Wyatt, 1986a, Quantum mechanical manifestations of cantori: wave-packet localization in stochastic regions, Phys. Rev. Lett. 57,1 .

Brown, R.C. and R.E. Wyatt, 1986b, Barriers to chaotic classical motion and quantum mechanical localization in multiphoton dissociation, J. Phys. Chem. $90,3590$.

Bruehl, M. and G.C. Schatz, 1988, Theoretical studies in collisional energy transfer in highly excited molecules: the importance of intramolecular vibrational redistribution in successive collision modeling of $\mathrm{He}+\mathrm{CS}_{2}$, J. Chem. Phys. 89, 770.

Brumer, P., 1981, Intramolecular energy transfer: theories for the onset of statistical behavior, Adv. Chem. Phys. 47, 201.

Brumer, P. and M. Shapiro, 1980, Intramolecular dynamics: time evolution of superposition states in the regular and irregular spectrum, Chem. Phys. Lett. 72, 528.

Brumer, P. and M. Shapiro, 1985, Theoretical aspects of photodissociation and intramolecular dynamics, Adv. Chem. Phys. 60, 371.

Buch, V., R.B. Gerber and M.A. Ratner, 1984, A statistical wave function model for C-H/C-D overtone linewidths: application to $C_{6} H_{6}, C_{6} D_{6}$, $\mathrm{C}_{6} \mathrm{HD}_{5}, \mathrm{C}_{6} \mathrm{HF}_{5}$, J. Chem. Phys. 81, 3393.

Budimir, J. and J.L. Skinner, 1987, On the relationship between $T_{1}$ and $T_{2}$ for stochastic relaxation models, J. Stat. Phys. 49 , 1029.

Bunker, D.L., 1962, Monte Carlo calculation of triatomic dissociation rates, I. $\mathrm{N}_{2} \mathrm{O}$ and $\mathrm{O}_{3}$, J. Chem. Phys. $37,393$.

Burleigh, D.C., R.C. Mayrhofer and E.L. Sibert III, 1988, Rotation-vibration interactions between the two lowest frequency modes in formaldehyde, J. Chem. Phys. 89, 7201. 
Butler, J.N. and G.B. Kistiakowski, 1960, Reactions of methylene. IV. Propylene and cyclopropane, J. Am. Chem. Soc. 82, 759.

Butler, L.J., E.J. Hintsa and Y.T. Lee, 1986a, Bond selective photochemistry in $\mathrm{CH}_{2} \mathrm{BrI}$ through electronic excitation at $210 \mathrm{~nm}$, J. Chem. Phys. $84,4104$.

Butler, L.J., T.M. Ticich, M.D. Likar, and F.F. Crim, 1986b, Vibrational overtone spectroscopy of bound and predissociative states of hydrogen peroxide cooled in a supersonic expansion, J. Chem. Phys. 85, 2331.

Butler, L.J., E.J. Hintsa, S.F. Shane, and Y.T. Lee, 1987, The electronic state-selective photodissociation of $\mathrm{CH}_{2} \mathrm{BrI}_{\text {at }} 248,210$ and $193 \mathrm{~nm}$. J. Chem. Phys. 86, 2051.

Byrne, J.P., E.F. McCoy and I.G. Ross, 1965, Internal conversions in aromatic and N-heteroaromatic molecules, Aust. J. Chem. $18,1589$.

Carmeli, B. and A. Nitzan, 1980a, Random coupling models for intramolecular dynamics. I. Mathematical approach, J. Chem. Phys. $72,2054$.

Carmeli, B. and A. Nitzan, 1980b, Random coupling models for intramolecular dynamics. II. Kinetic equations for collisionless multiphoton excitation of large molecules, J. Chem. Phys. 72, 2070.

Carmeli, B., I. Scheck, A. Nitzan and J. Jortner, 1980, Numerical simulations of molecular multiphoton excitation models, J. Chem. Phys. $72,1928$.

Carter, D. and P. Brumer, 1982, Intramolecular dynamics and nonlinear mechanics of model OCS, J. Chem. Phys. 77, 4208.

Carter, D. and P. Brumer, 1983, erratum: Intramolecular dynamics and nonlinear mechanics of model OCS [J. Chem. Phys. 77, 4208 (1982)], J. Chem. Phys. 78, 2104.

Casartelli, M., 1983, Relaxation time and randomness in phase space, Nuovo Cimento B 76, 1983.

Cassassa, M.P., D.S. Bomse and K.C. Janda, 1981, Infrared photodissociation of Van der Waals molecules containing ethylene, J. Chem. Phys. 74, 5044.

Cerjan, C. and W.P. Reinhardt, 1979, Critical point analysis of instabilities in Hamiltonian systems: classical mechanics of stochastic intramolecular energy transfer, J. Chem. Phys. 71, 1819.

Chaiken, J., T. Benson, M. Gurnick and J.D. McDonald, 1979, Quantum beats in single rovibronic state fluorescence of biacetyl, Chem. Phys. Lett. 61,195

Chang, J., N. Moiseyev and R.E. Wyatt, 1986, Stable highly excited vibrational eigenvalues without the variational principle, J. Chem. Phys. 84, 4997.

Chapman, S., B.C. Garrett and W.H. Miller, 1976, Semiclassical eigenvalues for nonseparable systems: nonperturbative solution of the Hamilton-Jacobi equation in action-angle variables, J. Chem. Phys. 64, 502.

Child, M.S. and L.O. Halonen, 1984, Overtone frequencies and intensities in the local mode picture, Adv. Chem. Phys. 57, 1.

Chirikov, B.V., 1959, The passage of a nonlinear oscillating system through resonance, Sov. Phys. - Dokl. 4, 390.

Chirikov, B.V., 1960, Resonance processes in magnetic traps, J. Nucl. Energy Part C: Plasma Phys., 1, 253.

Chirikov, B.V., 1979, A universal instability of many-dimensional oscillator systems, Phys. Rep. 52, 263.

Chirikov, B.V., 1983, in: Dynamical Systems and Chaos, ed. L. Garrido, (Springer, Berlin) p. 29.

Chirikov, B.V., F.M. Izrailev and D.L. Shepelyanski, Dynamical stochasticity in classical and quantum mechanics, Sov. Sci. Rev. C 2, 209.

Christoffel, K.B. and J.M. Bowman, 1982, Complex coordinate, self-consistent field calculations of vibrational resonance energies, J. Chem. Phys. 76,5370 .

Christoffel, K.B. and J.M. Bowman, 1983, Complex coordinate calculations of Feshbach resonance energies and widths for a collinear triatomic system, J. Chem. Phys. 78, 3952.

Chuang, M.-C., J.E. Baggott, D.W. Chandler, W.E. Farneth and R.N. Zare, 1983, Unimolecular decomposition of t-butyl hydroperoxide by direct excitation of the 6-0 O-H stretching overtone, Faraday Disc. Chem. Soc. 75, 301.

Chuljian, D.T., J. Ozment and J. Simons, 1984, Dissociation of vibronic states of C-'A' DCN: Quantum treatment, J. Chem. Phys. 80, 176.

Clarke, D.L. and M.A. Collins, 1987a, CH and CD overtone decay times in partially deuterated benzenes, J. Chem. Phys. $86,6871$.

Clarke, D.L. and M.A. Collins, 1987b, A classical trajectory study of the high $\mathrm{CH}$ and $\mathrm{CD}$ overtones in benzene and perdeuterobenzene, J. Chem. Phys. 87, 5312 .

Clodius, W.B. and R.B. Shirts, 1984, Enhancement of intramolecular vibrational energy transfer through rotational coupling, J. Chem. Phys. 81, 6224.

Colbert, D.T. and E.L. Sibert III, 1989, Variable curvature coordinates for molecular vibrations, J. Chem. Phys. $91,350$.

Collins, M.A., 1981, Solitons in chemical physics, Adv. Chem. Phys. 46, 73.

Contopoulos, G., 1971, Orbits in highly perturbed dynamical systems. III. Nonperiodic orbits, Astron. J. $76,147$.

Crim, F.F., 1984, Selective excitation studies of unimolecular reaction dynamics, Annu. Rev. Phys. Chem. 35, 657.

Crim, F.F., 1987, The dissociation dynamics of highly vibrationally excited molecules, in: Molecular Photodissociation Dynamics, eds. M.N.R. Ashfold and J.E. Baggott eds (Royal Society of Chemistry, London) p. 177.

Couley, R.A., 1963, The lattice dynamics of an anharmonic crystal, Adv. Phys. 12, 421.

Coveleskie, R.A., D.A. Dolson and C.S. Parmenter, 1985a, Chemical timing. 1. A method of picosecond fluorescence spectroscopy of intramolecular vibrational distribution, J. Phys. Chem. 89, 645.

Coveleskie, R.A., D.A. Dolson and C.S. Parmenter, 1985b, Chemical timing. 2. The picosecond dynamics of intramolecular vibrational redistribution in $S_{1}$ p-difluorobenzene, J. Phys. Chem. 89, 655.

Dai, H.-L., R.W. Field and J.L. Kinsey, 1985a, Intramolecular vibrational dynamics including rotational degrees of freedom: chaos and quantum spectra, J. Chem. Phys. 82, 2161.

Dai, H.-L., C.L. Korpa, J.L. Kinsey, and R.W. Field, 1985b, Rotation-induced vibrational mixing in $\mathrm{X}^{1} \mathrm{~A}_{1}$ formaldehyde: non-negligible dynamical consequences of rotation, J. Chem. Phys, 82, 1688. 
Davis, M.J., 1984, Exponential separation and long-time correlation in collinear OCS, Chem. Phys. Lett. 110, 491.

Davis, M.J., 1985, Bottlenecks to intramolecular energy transfer and the calculation of relaxation rates, J. Chem. Phys. 83, 1016.

Davis, M.J., 1987, Phase space dynamics of bimolecular reactions and the breakdown of transition state theory, J. Chem. Phys. $86,3978$.

Davis, M.J.. 1988, Phase space structure in classically chaotic regions and the nature of quantum eigenstates, J. Phys. Chem. $92,3124$.

Davis, M.J. and S.K. Gray, 1986, Unimolecular reactions and phase space bottlenecks, J. Chem. Phys. 84, 5389.

Davis, M.J. and E.J. Heller, 1981, Quantum dynamical tunneling in bound states, J. Chem. Phys. 75, 246.

Davis, M.J. and E.J. Heller, 1984, Comparisons of classical and quantum dynamics for initially localized states, J. Chem. Phys. $80,5036$.

Davis, M.J. and A.J. Wagner, 1984, in: Resonances in Electron-Molecule Scattering, Van der Waals Complexes and Reactive Chemical Dynamics, ACS Symp. Series 263, ed. D.G. Truhlar (American Chemical Society, Washington).

De Leon, N. and B.J. Berne, 1981, Intramolecular rate process: isomerization dynamics and the transition to chaos, J. Chem. Phys. $75,3495$.

De Leon, N. and B.J. Berne, 1982a, Reaction dynamics in an ergodic system: the siamese stadium billiard, Chem. Phys. Lett. $93,162$.

De Leon, N. and B.J. Berne, 1982b, Reaction dynamics in a non-ergodic system: the siamese stadium billiard, Chem. Phys. Lett. $93,169$.

De Leon, N. and M.A. Mehta, 1988, Semiclassical spectral quantization: molecular energies and eigenfunctions, Comput. Phys. Rep. 8. 293.

De Leon, N., M.J. Davis and E.J. Heller, 1984, Quantum manifestations of classical resonance zones, J. Chem. Phys. 80, 794.

Delos, J.B., 1986, Semiclassical calculation of quantum mechanical wavefunctions, Adv. Chem. Phys. 65, 161.

Deprit, A., 1967, Free rotations of a rigid body studied in the phase plane, Am. J. Phys. 35, 424.

De Souza, A.M., D. Kaur and D.S. Perry, 1988, Eigenstate-resolved vibrational state mixing in jet-cooled 1-butyne near $3333 \mathrm{~cm}{ }^{-1}$, Ber. Bunsenges, Phys. Chem. 92, 424.

Dietz, W. and S.F. Fischer, 1987, Model studies for rovibronic IVR with applications to benzene, J. Chem. Phys. 87, 249.

Dolson, D.A., K.W. Holtzclaw, D.B. Moss and C.S. Parmenter, 1985, Chemical timing. 4. The rovibronic level structure associated with intramolecular vibrational redistribution in ${ }^{1} S$ p-difluorobenzene, J. Chem. Phys. 84, 1119.

Druger, S.D., 1977a, Resonance line shapes in the Rydberg spectra of large molecules, J. Chem. Phys. 67, 3238.

Druger, S.D., 1977b, Coupling-strength incoherence and the absorption line shape of an isolated resonance in a large molecule, J. Chem. Phys. 67, 3249.

Dumont, R.S. and P. Brumer, 1987, Relaxation rates in chaotic and quasiperiodic systems, J. Chem. Phys. 87, 6437.

Dumont, R.S. and P. Brumer, 1988, Nonstatistical unimolecular decay in quasiperiodic systems, J. Chem. Phys. 90, 96.

Dumont, R. and P. Pechukas, 1988, Semiclassical localization in a one-dimensional random analytic potential, J. Chem. Phys. 89, 5764.

Duneczky, C. and W.P. Reinhardt, 1990, Vibrational relaxation from high $\mathrm{CH}$ overtones in butane, preprint.

Eastes, W. and R.A. Marcus, 1974, Semiclassical calculation of bound states of a multidimensional system, J. Chem. Phys. $61,4301$.

Eckhardt, B., 1988, Quantum mechanics of classically nonintegrable systems, Phys. Rep. 163, 205.

Escande, D.F., 1985, Stochasticity in classical Hamiltonian systems: universal aspects, Phys. Rep. 121, 165.

Escande, D.F. and F. Doveil, 1981, Renormalization method for computing the threshold of the large-scale stochastic instability in two degrees of freedom Hamiltonian systems, J. Stat. Phys. 26, 257.

Evard, D.D., C.R. Bieler, J.I. Cline, N. Sivakumar and K.C. Janda, 1988, The vibrational predissociation dynamics of $\mathrm{ArCl}_{2}$ : intramolecular vibrational relaxation in a triatomic Van der Waals molecule?, J. Chem. Phys. 89, 2829.

Ewing, G.E., 1987, Selection rules for vibrational energy transfer: vibrational predissociation of Van der Waals molecules, J. Phys. Chem. 91, 4662.

Ewing, G.E., R.J. Longfellow, D.B. Moss and C.S. Parmenter, 1987, Van der Waals coupling between internal rotation and molecular vibrations. The methyl rotor effect on IVR, in: Stochasticity and Intramolecular Redistribution of Energy, eds. R. Lefebvre and S. Mukamel (Reidel. Dordrecht) p. 163.

Ezra, G.S. 1982, Symmetry Properties of Molecules, Lecture Notes in Chemistry No. 28 (Springer, New York)

Ezra, G.S., 1986, Interaction between bending vibrations and molecular rotation: a model study. Chem. Phys. Lett. 127, 492.

Ezra, G.S., C.C. Martens and L.E. Fried, 1987, Semiclassical quantization of polyatomic molecules: some recent developments, J. Phys. Chem. 91, 3621.

Faid, K. and R.F. Fox, 1987, Stochastic theory of relaxation and approach to thermal equilibrium for phonon reservoirs, Phys. Rev. A $35,2684$.

Faid, K. and R.F. Fox, 1988, The absorption line shape for a molecular system stochastically coupled to a phonon thermal reservoir, J. Chem. Phys. $88,4579$.

Farantos, S.C. and N. Flytzanis, 1987, A classical mechanical analysis of vibrational dephasing and rotational energy redistribution in CO-Ar, J. Chem. Phys. 87, 6449 .

Farantos, S.C. and J. Tennyson, 1987, Chaos in molecular systems?, in: Stochasticity and Intramolecular Redistribution of Energy, eds R. Lefebvre and S. Mukamel (Reidel, Dordrecht) p. 15.

Farrelly, D., 1986a, Lie algebraic approach to quantization of nonseparable systems with internal nonlinear resonance, J. Chem. Phys. 85, 2119.

Farrelly, D., 1986b, Uniform semiclassical self-consistent-field and adiabatic calculations of complex energy eigenvalues for nonseparable systems, J. Chem. Phys. 84, 6285.

Farrelly, D. and A.D. Smith, 1986, A generalized self-consistent-field procedure for nonseparable vibrationally bound states, J. Phys. Chem. 90, 1599 .

Farrelly, D. and T. Uzer, 1986, Semiclassical quantization of slightly nonresonant systems: avoided crossings, Fermi resonances, and dynamical tunneling, J. Chem. Phys. 85,308

Felker, P.M. and A.H. Zewail, 1984, Direct picosecond time resolution of dissipative intramolecular vibrational energy redistribution (IVR) in isolated molecules, Chem. Phys. Lett. 108, 303. 
Felker, P.M. and A.H. Zewail, 1985a, Dynamics of intramolecular vibrational-energy redistribution (IVR). I. Coherence effects, J. Chem. Phys. 82, 2961.

Felker, P.M. and A.H. Zewail, 1985b, Dynamics of intramolecular vibrational-energy redistribution (IVR). II. Excess energy dependence, J. Chem. Phys. 82, 2975.

Felker, P.M. and A.H. Zewail, 1985c, Dynamics of intramolecular vibrational-energy redistribution (IVR). III. Role of molecular rotations, J. Chem. Phys. 82, 2994.

Felker, P.M. and A.H. Zewail, 1985d, Dynamics of intramolecular vibrational-energy redistribution (IVR). IV. Excess energy dependence, t-stilbene, J. Chem. Phys. 82, 3003.

Felker, P.M., W.R. Lambert and A.H. Zewail, 1982, Picosecond excitation of jet-cooled pyrazine: magnetic field effects on the fluorescence decay and quantum beats, Chem. Phys. Lett. 89, 309.

Fermi, E., 1923, Beweiss dass ein mechanisches Normalsystem im allgemeinen quasiergodisch ist, Z. Physik 24, 261.

Fermi, E., 1931, Uber den Ramaneffekt des Kohlendioxyds, Z. Physik 71, 250.

Fermi, E., J.R. Pasta and S.M. Ulam, 1955, Studies of nonlinear problems, Los Alamos Scientific Laboratory report LA-1940. Also in: Collected Works of Enrico Fermi, Vol. II (Univ. of Chicago Press, Chicago, 1965) p. 978.

Fischer, S.F. and W. Dietz, 1987, Rotation selective intramolecular vibrational relaxation in the $S_{1}$-state of benzene, in: Stochasticity and Intramolecular Redistribution of Energy, eds R. Lefebvre and S. Mukamel (Reidel, Dordrecht) p. 193.

Fischer, G., R.E. Miller and R.O. Watts, 1983, Vibrational predissociation of Van der Waals clusters of ethylene, Chem. Phys. 80, 147.

Florencio, Jr, J. and M.H. Lee, 1985, Exact time evolution of a classical harmonic-oscillator chain, Phys. Rev. A $31,3231$.

Ford, J., 1961, Equipartition of energy for nonlinear systems, J. Math. Phys. 2, 387.

Ford, J., 1973, The transition from analytic dynamics to statistical mechanics, Adv. Chem. Phys. 24, 155.

Ford, J., 1975, The statistical mechanics of classical analytic dynamics, in: Fundamental Problems in Statistical Mechanics, Vol. 3, ed. E.D.G. Cohen (North-Holland, Amsterdam).

Ford, J., 1978, A picture book of stochasticity, in: Topics in Nonlinear Dynamics, S. Jorna, ed. American Institute of Physics conference proceedings No. 46 (American Institute of Physics, New York) p. 121.

Ford, J. and G.H. Lunsford, 1970, Stochastic behavior of resonant nearly linear oscillator systems in the limit of zero nonlinear coupling, Phys. Rev. A 1,59 .

Ford, J. and J. Waters, 1963, Computer studies of energy sharing and ergodicity for nonlinear oscillator systems, J. Math. Phys. 4, 1293.

Forst, W., 1973, Theory of Unimolecular Reactions (Academic Press, New York).

Fox, R.F., 1978, Gaussian stochastic processes in physics, Phys. Rep. 48, 179.

Fox, R.F., 1989, Stochastic processes in quantum-mechanical settings, in: Noise in Nonlinear Systems, Vol. 2: Theory of Noise-Induced Processes in Special Applications, eds F. Moss and P.V.E. McClintock (Cambridge Univ. Press, New York) p. 1.

Foy, R.B., M.P. Casassa, J.C. Stephenson and D.S. King, 1989, Dissociation lifetimes and level mixing in overtone-excited $\mathrm{HN}_{3}\left(\tilde{\mathrm{X}}^{1} \mathrm{~A}^{\prime}\right)$, J. Chem. Phys. 90, 7037.

Frad, A., F. Lahmani, A. Tramer and C. Tric, 1974, Luminescence and radiationless transitions from single vibronic levels of the isolated pyrazine molecule in the $S_{1}\left(n, \pi^{*}\right)$ state, J. Chem. Phys. $60,4419$.

Frederick, J.H. and G.M. McClelland, 1986a, Nonlinear dynamics of vibration-rotation interactions: rigid bender $\mathrm{H}_{2} \mathrm{O}$, J. Chem. Phys. 84, 4347.

Frederick, J.H. and G.M. McClelland, 1986b, Semiclassical quantization of the vibration-rotation problem, J. Chem. Phys. $84,876$.

Frederick, J.H., G.M. McClelland and P. Brumer, 1985, Classical trajectory study of vibration-rotation interaction in highly excited triatomic molecules, J. Chem. Phys. 83, 190.

Frederick, J.H., E.J. Heller, J.L. Ozment and D.W. Pratt, 1988, Ring torsional dynamics and spectroscopy of benzophenone: a new twist, J. Chem. Phys. 88, 2169.

Freed, K.F., 1970, Irreversible electronic relaxation in polyatomic molecules, J. Chem. Phys. 52, 1345.

Freed, K.F., 1976, Energy dependence of electronic relaxation processes in polyatomic molecules, Topics Appl. Phys. 15, 23.

Freed, K.F., 1979, Remarks, Faraday Disc. Chem. Soc. 67, 231.

Freed, K.F., 1980, Collisional effects on electronic relaxation processes, Adv. Chem. Phys. 42, 207.

Freed, K.F., 1981, Collision-induced intersystem crossing, Adv. Chem. Phys. 47 (Part 2) 291.

Freed, K.F. and A. Nitzan, 1980, Intramolecular vibrational energy redistribution and the time evolution of molecular fluorescence, J. Chem. Phys. 73,4765 .

Freed, K.F. and A. Nitzan, 1983, Theoretical analysis of experimental probes of dynamics of intramolecular vibrational relaxation, in: Energy Storage and Redistribution in Molecules, ed. J. Hinze (Plenum, New York) p. 467.

Frye, D., L. Lapierre and H.-L. Dai, 1988, Vibration-rotation spectroscopy by stimulated emission on pumping in a supersonic beam: $a$-axis Coriolis coupled $\nu_{4}=1$ and $\nu_{8}=1$ levels of $\mathrm{X}^{1} \mathrm{~A}_{\mathrm{g}}$ glyoxal, J. Chem. Phys. 89, 2609.

Gadzuk, J.W., 1987, Intramolecular dynamics in molecule-surface collisions: excitation, dissociation and selectivity of reactivity, Surf. Sci. $184,483$.

Gadzuk, J.W., 1988, The semiclassical way to molecular dynamics at surfaces, Annu. Rev. Phys. Chem. 39, 463.

Garcia-Ayllon, A., J. Santamaria and G.S. Ezra, 1988, Sensitivity of intramolecular vibrational energy relaxation to stretch-bend potential energy coupling and stability of periodic orbits, J. Chem. Phys. 89,801 .

Garcia-Colin, L.S. and J.L. del Rio, 1977, A unified approach for deriving kinetic equations in nonequilibrium statistical mechanics. I. Exact results, J. Stat. Phys. 16, 235. 
Garland, N.L. and E.K.C. Lee, 1986, Coriolis-induced intensity perturbations in the rotationally resolved fluorescence spectra from the $5^{i}$ and $1^{i} 4^{1}$ eigenstates of $\mathrm{H}_{2} \mathrm{CO}\left(\mathrm{A}^{\prime} \mathrm{A}, \mathrm{s}\right)$ : vibrational mixing near $E_{\mathrm{vit}}=3000 \mathrm{~cm}^{-1}, \mathrm{~J}$. Chem. Phys. $84,28$.

Garland, N.L., E.C. Apel and E.K.C. Lee, 1983, Role of rotation-vibration interaction in vibrational relaxation: energy redistribution in excited singlet formaldehyde, Faraday Disc. Chem. Soc. 75, 377.

Geisel, T., G. Radons and J. Rubner, 1986, Kolmogorov-Arnol'd-Moser barriers in the quantum dynamics of chaotic systems, Phys. Rev. Lett. 57, 2883.

Gelbart, W.M. and K.F. Freed, 1973, Intramolecular perturbations and the quenching of luminescence in small molecules, Chem. Phys. Lett. 18, 470.

Gelbart, W.M., D.F. Heller and M.L. Elert, 1975, Decays, recurrences and large molecule behavior in the theory of radiationless transitions, Chem. Phys. 7, 116.

Gerber, R.B. and M.A. Ratner, 1988, Mean-field models for molecular states and dynamics: new developments, J. Phys. Chem. $92,3252$.

Getino, C., B.G. Sumpter, J. Santamaria and G.S. Ezra, 1989, Quasiclassical overtone linewidths and unimolecular decay lifetimes for HOOH, J. Phys. Chem. 93, 3877.

Gibson, L.L., G.C. Schatz and M.A. Ratner, 1987, Phase space bottlenecks: a comparison of quantum and classical intramolecular dynamics for collinear OCS, J. Chem. Phys. 86, 3263.

Gilbert, R.G., 1984, Collisional energy exchange in highly vibrationally excited molecules: the biased random walk model, J. Chem. Phys. 80, 5501.

Glasstone, S., K.J. Laidler and H. Eyring, 1941, The Theory of Rate Processes (McGraw-Hill, New York).

Goldberger, M.L. and R.M. Watson, 1969, Collision Theory (Wiley, New York).

Goldstein, H., 1980, Classical Mechanics, 2nd Ed. (Addison-Wesley, New York).

Gole, J.L., 1985, Probing ultrafast energy transfer among the excited state of small high temperature molecules, in: Gas-Phase Chemiluminescence and Chemi-ionization, ed. A. Fontijn (Elsevier, Amsterdam) p. 253.

Gordon, R.J., 1988, Recent trends in experimental studies of vibrational energy transfer, Comments At. Mol. Phys. 21, 123.

Grad, J., Y.J. Yan, A. Hague and S. Mukamel, 1987, Reduced equations of motion for semiclassical dynamics in phase space, J. Chem. Phys. 86, 3441

Graffi, S., T. Paul and H.J. Silverstone, 1987, Classical resonance overlapping and quantum avoided crossings, Phys. Rev. Lett. $59,255$.

Graffi, S., T. Paul and H.J. Silverstone, 1988, Resonance overlapping in classical mechanics and avoided crossings in quantum mechanics, Phys. Rev. A 37, 2214

Graner, G., 1988, Can high-resolution molecular spectroscopy help to understand intramolecular energy transfers?, Ber. Bunsenges. Phys. Chem. $92,381$.

Gray, S.K. and M.S. Child, 1984, The vibrational energy states of two coupled modes in formaldehyde. Classical, quantum, and semiclassical analysis, Mol. Phys. 53, 961.

Gray, S.K. and M.J. Davis, 1989, Coriolis induced vibration and rotation mixing in formaldehyde, J. Chem. Phys. 90, 5420.

Gray, S.K. and S.A. Rice, 1987, Phase space bottlenecks and statistical theories of isomerization reactions, J. Chem. Phys. 86, 2020.

Gray, S.K., J.R. Stine and D.W. Noid, 1985, Classical trajectory calculations of infrared multiphoton absorption and dissociation, Laser Chem. 5 , 209.

Gray, S.K., S.A. Rice and D.W. Noid, 1986a, The classical mechanics of vibrational predissociation: a model study of phase space structure and its influence on fragmentation rates, J. Chem. Phys. 84, 3745.

Gray, S.K., S.A. Rice and M.J. Davis, 1986b, Bottlenecks to unimolecular reactions and an alternative form for classical RRKM theory, J. Phys. Chem. 90, 3470 .

Green Jr., W.H., W.D. Lawrance and C.B. Moore, 1987, Kinetic anharmonic coupling in the trihalomethanes: a mechanism for rapid intramolecular redistribution of $\mathrm{CH}$ stretch vibrational energy, J. Chem. Phys. 86, 6000.

Greene, J.M., 1968, Two-dimensional measure-preserving maps, J. Math. Phys. 9, 760.

Greene, J.M., 1979, A method for determining a stochastic transition, J. Math. Phys. 20, 1183.

Guan, Y., G.C. Lynch and D.L. Thompson, 1987, Intramolecular energy transfer and cis-trans isomerization in HONO, J. Chem. Phys. 87, 6957.

Gutow, J.H., D. Klenerman and R.N. Zare, 1988, Comparison of overtone-induced and electronic photochemistry of liquid t-butyl hydroperoxide: supporting evidence for vibrational mode-specificity, J. Phys. Chem. 92, 172.

Ha, J.M.Y. H.J. Maris, W.M. Risen, Jr, J. Tauc, C. Thomsen and Z. Vardeny, 1986, Observation of molecular vibrations in real time, Phys. Rev. Lett. 57,3302 .

Hamilton, 1. and P. Brumer, 1983, Intramolecular relaxation in $N=2$ Hamiltonian systems: the role of the $K$ entropy, J. Chem. Phys. $78,2682$.

Hamilton, I., D. Carter and P. Brumer, 1982, Relaxation rates in model Hamiltonian systems, with remarks on OCS. J. Phys. Chem. 86, 2124.

Hamilton, C.E., J.L. Kinsey and R.W. Field, 1986, Stimulated emission pumping: new methods in spectroscopy and molecular dynamics, Annu. Rev. Phys. Chem. 37, 493.

Hammel, S.M., J.A. Yorke and C. Grebogi, 1987, Do numerical orbits of chaotic dynamical processes represent true orbits? J. Complexity $3,136$. Hanson, J.D., J.R. Cary and J.D. Meiss, 1985, Algebraic decay in self-similar Markov chains, J. Stat. Phys. $39,327$.

Harris, A.L. and N.J. Levinos, 1989, Vibrational energy relaxation in a molecular monolayer at a metal surface, J. Chem. Phys. $90,3878$.

Harter, W.G., 1984, Patterns of energy levels and spectra for polyatomic molecules, J. Stat. Phys. 36, 749.

Harter, W.G., 1986, SU(2) coordinate geometry for semiclassical theory of rotors and oscillators, J. Chem. Phys. 85, 5560.

Harter, W.G., 1988, Computer graphical and semiclassical approaches to molecular rotations and vibrations, Comput. Phys. Rep. $8,319$. 
Harter, W.G. and C.W. Patterson, 1984, Rotational energy surfaces and high-J eigenvalue structure of polyatomic molecules, J. Chem. Phys. 80, 4241.

Hase, W.L., 1976, Dynamics of unimolecular reactions, in: Modern Theoretical Chemistry, Vol. 2, ed. W.H. Miller (Plenum, New York) p. 121.

Hase, W.L., 1981, Overview of unimolecular dynamics, in: Potential Energy Surfaces and Dynamics Calculations (Plenum, New York).

Hase, W.L., 1982, Semiclassical vibrational energy levels for a model H-C-C $\rightarrow \mathrm{H}+\mathrm{C}=\mathrm{C}$ Hamiltonian, J. Phys. Chem. 86, 2873.

Hase, W.L., 1983, Variational unimolecular rate theory, Acc. Chem. Res. 16, 258.

Hase, W.L., 1985, On non-exponential unimolecular dissociation of molecules prepared by vibrational overtone excitation, Chem. Phys. Lett. 116, 312.

Hase, W.L., 1986, Unimolecular and intramolecular dynamics: relationship to potential energy surface properties, J. Phys. Chem. $90,365$.

Hase, W.L. and D.G. Buckowski, 1982, Dynamics of ethyl radical decomposition. II. Applicability of classical mechanics to large-molecule unimolecular reaction dynamics, J. Comput. Chem. 3, 335.

Hase, W.L., D.G. Buckowski and K.N. Swamy, 1983, Dynamics of ethyl radical decomposition. III. Effect of chemical activation versus microcanonical sampling, J. Phys. Chem. 87, 2754.

Hase, W.L., R.J. Duchovic, K.N. Swamy and R.C. Wolf, 1984, Trajectory studies of model H $-\mathrm{C}-\mathrm{C} \rightarrow \mathrm{H}+\mathrm{C}=\mathrm{C}$ dissociation. III. Details of the lifetime distribution following chemical activation, J. Chem. Phys. 80, 714

Haub, J.G. and B.J. Orr, 1987, Coriolis-assisted vibrational energy transfer in $\mathrm{D}_{2} \mathrm{CO} / \mathrm{D}_{2} \mathrm{CO}$ and $\mathrm{HDCO} / \mathrm{HDCO}$ collisions: experiment and theory, J. Chem. Phys. 86, 3380.

Hedges, R.M. and W.P. Reinhardt, 1983, Classical and quantum dynamics of long lived doubly excited states of triatomic molecules, J. Chem. Phys. 78,3964 .

Heidberg, J., H. Stein, E. Riehl, Z. Szilagy and H. Weiss, 1985, Vibrational predesorption, Surf. Sci. 158, 553.

Heidberg, J., H. Stein and W. Weiss, 1987, Vibrational predesorption of carbon monoxide from sodium chloride at $20 \mathrm{~K}$ induced by resonant infrared laser excitation, Surf. Sci. 184, L 431.

Heilweil, E.J., R.R. Cavanagh and J.C. Stephenson, 1988a, Picosecond study of the population lifetime of $\mathrm{CO}(v=1)$ chemisorbed on $\mathrm{SiO}_{2}$-supported rhodium particles, J. Chem. Phys. 89, 5342.

Heilweil, E.J., J.C. Stephenson and R.R. Cavanagh, 1988b, Measurements of CO $(v=1)$ population lifetimes: metal-carbonyl cluster compounds supported on $\mathrm{SiO}_{2}$, J. Phys. Chem. 92, 6099.

Heller, D.F. and S. Mukamel, 1979, Theory of vibrational overtone line shapes of polyatomic molecules, J. Chem. Phys. $70,463$.

Heller, D.F., M.L. Elert and W.M. Gelbart, 1978, Photodissociation dynamics of small polyatomics: isolated molecule behavior and collision induced effects, J. Chem. Phys. 69, 4061.

Heller, E.J., 1980, Quantum intramolecular dynamics: criteria for stochastic and nonstochastic flow, J. Chem. Phys. $72,1337$.

Heller, E.J., 1981a, The semiclassical way to molecular spectroscopy, Acc. Chem. Res. 14, 368.

Heller, E.J., 1981b, in: Potential Energy Surfaces and Dynamics Calculations for Chemical Reactions and Molecular Energy Transfer, ed. D.G. Truhlar (Plenum Press, New York) p. 103.

Heller, E.J., 1983, The correspondence principle and intramolecular dynamics, Faraday Disc. Chem. Soc. 75, 141.

Heller, E.J., 1987, Quantum localization and the rate of exploration of phase space, Phys. Rev. A 35, 1360.

Heller, E.J. and M.J. Davis, 1981, Quantum dynamical tunneling in large molecules. A plausible conjecture, J. Phys. Chem. 85, 307.

Heller, E.J. and S.A. Rice, 1974, Random coupling model for molecular dissociation, J. Chem. Phys. 61, 936.

Heller, E.J., E.B. Stechel and M.J. Davis, 1979, Molecular spectra, Fermi resonances, and classical motion: example of $\mathrm{CO}_{2}$, J. Chem. Phys. 71, 4759.

Heller, E.J., E.B. Stechel and M.J. Davis, 1980, Molecular spectra, Fermi resonances, and classical motion, J. Chem. Phys. 73, 4720.

Hénon, M. and C. Heiles, 1964, The applicability of the third integral of motion: some numerical experiments, Astron. J. $69,73$.

Henry, B.I. and J. Grindlay, 1986, Numerical ensemble study of the approach to equilibrium of an anharmonic chain, Phys. Lett. A $119,215$.

Henry, B.I. and J. Grindlay, 1987, Reversible approach to statistical equilibrium in a nonlinear chain: an ensemble study, Physica D $28,49$.

Henry, B.I. and J. Grindlay, 1988, Numerical ensemble study of ergodic properties of the quartic Fermi-Pasta-Ulam chain, Phys. Rev. A 38, 2594.

Henry, B.I. and J. Oitmaa, 1983, Dynamics of a nonlinear diatomic chain, Aust. J. Phys. 36, 339.

Henry, B.I. and J. Oitmaa, 1985a, Dynamics of a nonlinear diatomic chain. II. Thermodynamic properties, Aust. J. Phys. 38, 171.

Henry, B.I. and J. Oitmaa, 1985b, Dynamics of a nonlinear diatomic chain. III. A molecular dynamics study, Aust. J. Phys. 38, 191.

Henry, B.R., 1977, Use of local modes in the description of highly vibrationally excited molecules, Acc. Chem. Res. 10, 207.

Heppener, M., A.G.M. Kunst, D. Bebelaar and P.H. Rettschnick, 1985, Time-resolved mode-to-mode vibrational energy flow in the isolated s-tetrazine argon complex, J. Chem. Phys. 83, 5341.

Hiroike, E. and Y. Fujimura, 1988, Simultaneous interaction of Coriolis and anharmonic coupling in the intramolecular vibrational redistribution of polyatomic molecules, Chem. Phys. Lett. 143, 193.

Hoffbauer, M.A., K. Liu, C.F. Giese and W.R. Gentry, 1983, The dynamics of ethylene dimer infrared photodissociation in pulsed molecular beams, J. Chem. Phys. 78, 5567.

Hofmann, P., R.B. Gerber, M.A. Ratner, L.C. Baylor and E. Weitz, 1988, Dynamics of energy flow from CH overtone excitations: theoretical and experimental studies of $\mathrm{CH}_{3} \mathrm{C}=\mathrm{CH}$, J. Chem. Phys. 88, 7434 .

Holme, T.A. and J.S. Hutchinson, 1985, Vibrational energy flow into a reactive coordinate: a theoretical prototype for a chemical system, J. Chem. Phys. 83, 2860. 
Holme, T.A. and J.S. Hutchinson, 1986, Dynamics of overtone excitation for a polyatomic model, J. Chem. Phys. $84,5455$.

Holme, T.A. and R.D. Levine, 1988a, Energy flow pathways and their spectral signatures in vibrationally excited acetylene, J. Chem. Phys. 89, 3379.

Holme, T.A. and R.D. Levine, 1988b, Short-time vibrational dynamics of acetylene versus its isotropic variants, Chem. Phys. Lett. $150,293$.

Holme, T.A. and R.D. Levine, 1989, Theoretical and computational studies of highly vibrationally excited acetylene, Chem. Phys. $131,169$.

Holtzclaw, K.W. and C.S. Parmenter, 1986, Chemical timing 3. The picosecond dynamics of intramolecular vibrational redistribution from 11 levels in $S_{1}$ p-difluorobenzene vapor, J. Chem. Phys. 84, 1099.

Holtzclaw, K.W. and D.W. Pratt, 1986, Prominent, and restricted, vibrational state mixing in the fluorescence excitation spectrum of benzophenone, J. Chem. Phys. 84, 4713.

Hose, G. and H.S. Taylor, 1984, Mode localization in highly excited vibrational states: fundamentals of structure in overtone and multiphoton spectra, Chem. Phys. 84, 375.

Huber, D.A. and E.J. Heller, 1988, Hybrid mechanics, a combination of classical and quantum mechanics, J. Chem. Phys. $89,4752$.

Huber, D., E.J. Heller and R.G. Littlejohn, 1988, Generalized wavepacket dynamics, Schrödinger equation and stationary phase approximation, J. Chem. Phys. 89, 2003.

Hunt, G.R., E.F. McCoy and I.G. Ross, 1962, Excited states of aromatic hydrocarbons: pathways of internal conversion, Aust. J. Chem. 15, 591.

Hutchinson, J.S., 1987, The role of mode-mode energy flow in a model of the 1,5 hydrogen shift isomerization of malonaldehyde, J. Phys. Chem. $91,4495$.

Hutchinson, J.S., 1989, Local mode overtones and mode selectivity, Adv. Chem. Phys. 73, 637.

Hutchinson, J.S. and K.T. Marshall, 1988, Local mode overtones: ultrashort pulse excitation, intramolecular relaxation, and unimolecular reactions. J. Chem. Soc. Faraday Trans. 2, 84, 1535.

Hutchinson, J.S., W.P. Reinhardt and J.T. Hynes, 1983, Nonlinear resonances and vibrational energy flow in model hydrocarbon chains, J. Chem. Phys. 79, 4247.

Hutchinson, J.S., J.T. Hynes and W.P. Reinhardt, 1984, Quantum dynamic analysis of energy transfer in model hydrocarbons, Chem. Phys. Lett. $108,353$.

Hutchinson, J.S., J.T. Hynes and W.P. Reinhardt, 1986, A mechanism for local HC vibrational relaxation in alkanes, J. Phys. Chem. $90,3528$.

Imre, D., J.L. Kinsey, A. Sinha and J. Krenos, 1984, Chemical dynamics studied by emission spectroscopy of dissociating molecules, J. Phys. Chem. 88, 3956.

Ionov, S.I., A.A. Stuchebryukhov, V.N. Bagratashvili, V.N. Lokhman, G.N. Makarov and V.S. Letokhov, 1988, Evolution of the homogeneous IR spectrum of a highly vibrational excited molecule as a result of a change in its vibrational energy, Appl. Phys. B 47, 229.

Ito, M., 1988, Large amplitude motion and its role in the relaxation process, Ber. Bunsenges. Phys. Chem. 92, 345.

Izrailev, F.M., 1980, Nearly linear mappings and their applications, Physica D 1, 243.

Izrailev, F.M. and B.V. Chirikov, 1966, Statistical properties of a nonlinear string, Sov. Phys. - Dokl. 11, 30.

Jackson, E.A., 1963a, Nonlinear coupled oscillators. I. Perturbation theory, ergodic problem, J. Math. Phys. 4, 551.

Jackson, E.A., 1963b, Nonlinear coupled oscillators. II. Comparison of theory with computer solutions, J. Math. Phys. 4, 686.

Jaeger, F. and A.J. Lichtenberg, 1972, Resonant modification and destruction of adiabatic invariants, Ann. Phys. 71, 319.

Jaffé, C., 1988, Comment on semiclassical phase space evolution of Fermi resonance spectra, J. Chem. Phys. 89, 3395.

Jaffé, C. and P. Brumer, 1980, Local and normal modes: a classical perspective, J. Chem. Phys. 73, 5646.

Jaffé, C. and P. Brumer, 1984, Classical Liouville mechanics and intramolecular relaxation dynamics, J. Phys. Chem. 88, 4829.

Jaffé, C. and P. Brumer, 1985, Classical-quantum correspondence in the distribution dynamics of integrable systems, J. Chem. Phys. 82, 2330.

Jaffé, C. and W.P. Reinhardt, 1982, Uniform semiclassical quantization of regular and chaotic classical dynamics on the Hénon-Heiles surface, J. Chem. Phys. 77, 5191.

Jasinski, J.M., J.K. Frisoli and C.B. Moore, 1983, Unimolecular reactions induced by vibrational overtone excitation, Faraday Disc. Chem. Soc. 75 , 289.

Jasinski, J.M., J.K. Frisoli and C.B. Moore, 1984, High vibrational overtone photochemistry of 1-cyclopropylcyclobutene, J. Phys. Chem. 87, 3826. Jedrzejek, C., 1985, Selective laser-stimulated desorption of molecules by internal vibration excitation, J. Vac. Sci. Technol. B 3, 1431.

Jortner, J. and R.D. Levine, 1981, Photoselective chemistry, Adv. Chem. Phys. 47 (Part 1), 1.

Jucks, K.W. and R.E. Miller, 1987, The effect of vibrational state mixing on the predissociation lifetime of $\nu_{1}$ excited OC-HF, J. Chem. Phys. 86, 6637.

Kable, S.H. and A.E.W. Knight, 1987, Stimulated emission pumping of p-difluorobenzene cooled in a supersonic free jet. Vibrational relaxation in $\mathrm{S}_{0}$ induced by very low energy collisions, J. Chem. Phys. 86,4709 .

Kable, S.H., J.W. Thoman, Jr and A.E.W. Knight, 1988, Collision partner and level dependence of vibrational relaxation in $\mathrm{S}_{11} \mathrm{p}$-diffuorobenzene. Stimulated emission pumping combined with single vibronic level fluorescence spectroscopy, J. Chem. Phys. 88, 4748.

Karney, C.F.F., 1983, Long-time correlations in the stochastic regime, Physica D 8, 360.

Kato, S., 1985a, Intramolecular dynamics of the unimolecular dissociation of $\mathrm{CS}_{2}$ : analysis of the classical phase space structure, J. Chem. Phys. 82, 3020 .

Kato, S., 1985b, Intramolecular vibrational energy redistribution in $\mathrm{CHCl}_{3}$ : a theoretical analysis, J. Chem. Phys. $83,1085$.

Kay, K.G., 1974, Theory of vibrational relaxation in isolated molecules, J. Chem. Phys. 61, 5205.

Kay, K.G., 1976, Dynamical treatment of unimolecular decomposition reactions. The RRKM formula, J. Chem. Phys. 64,2112 
Kay, K.G., 1978, Dynamical treatment of unimolecular decomposition reactions II. Short-range interfragment coupling and incomplete randomization, J. Chem. Phys. 69, 434.

Kay, K.G., 1980, Numerical study of intramolecular vibrational energy transfer: Quantal, classical and statistical behavior, J. Chem. Phys. $72,5955$.

Kay, K.G., 1981, The Bloch equation for multiphoton absorption. I. Derivation, J. Chem. Phys. 75, 1690.

Kay, K.G., 1983, Toward a comprehensive semiclassical ergodic theory, J. Chem. Phys. 79, 3026.

Kay, K.G. and B. Ramachandran, 1988, Classical and quantal pseudoergodic regions of the Hénon-Heiles system, J. Chem. Phys. 88, 5688.

Kay, K.G., J. Stone, E. Thiele and M.F. Goodman, 1981, Derivation of the master equation by restricted quantum exchange, Chem. Phys. Lett. $82,539$.

Kellman, M.E., 1982, Group theory of coupled oscillators: Normal modes as symmetry breaking, J. Chem. Phys. 76, 4528.

Kellman, M.E., 1985, Algebraic resonance dynamics of the normal/local transition from experimental spectra of the ABA triatomics, J. Chem. Phys. 83, 3843.

Kellman, M.E., 1986, On the equivalence of the normal and local mode representations, J. Chem. Phys. 85, 6242.

Kellman, M.E. and E.D. Lynch, 1986a, Algebraic resonance quantization of coupled anharmonic oscillators, J. Chem. Phys. 85, 5855.

Kellman, M.E. and E.D. Lynch, 1986b, Fermi resonance phase space structure from experimental spectra, J. Chem. Phys. 85, 7216.

Kellman, M.E. and E.D. Lynch, 1988a, Semiclassical phase space evolution of Fermi resonance spectra, J. Chem. Phys. 88, 2205.

Kellman, M.E. and E.D. Lynch, 1988b, Reply to Comment on Semiclassical phase space evolution of Fermi resonance spectra, J. Chem. Phys. 89, 3396.

Kim, H.L., T.J. Kulp and J.D. McDonald, 1987, Infrared fluorescence study on the threshold of intramolecular vibrational state mixing, J. Chem. Phys. $87,4376$.

Klippenstein, S.J., G.A. Voth and R.A. Marcus, 1986, Iteratively determined effective Hamiltonians for the adiabatically reduced coupled equations approach to intramolecular dynamics calculations, J. Chem. Phys. 85, 5019.

Kneba, M. and J. Wolfrum, 1980, Bimolecular reactions of vibrationally excited molecules, Annu. Rev. Phys. Chem. $31,47$.

Knight, A.E.W., 1988a, IVR viewed through state-selected dispersed fluorescence spectroscopy of jet-cooled polyatomics, Ber. Bunsenges. Phys. Chem. 92, 337.

Knight, A.E.W., 1988b, Rotational involvement in intramolecular vibrational redistribution, Excited States $7,1$.

Kolmogorov, A.N., 1954, Dokl. Akad. Nauk. SSSR 98, 527.

Kommandeur, J., W.A. Majewski, W.L. Meerts and D.W. Pratt, 1987, Pyrazine: an "exact" solution to the problem of radiationless transitions, Annu. Rev. Phys. Chem. 38, 433.

Kommandeur, J., W.L. Meerts, Y.M. Engel and R.D. Levine, 1988, The analysis of intensity fluctuations for a fully resolved spectrum: pyrazine, J. Chem. Phys. 88, 6810.

Konings, J.A., W.A. Majewski, Y. Matsumoto, D.W. Pratt and W.L. Meerts, 1988, Ultra high-resolution fluorescence excitation spectrum of ${ }^{1} B_{1}$ pyrimidine in a molecular beam. Structural assignments, analysis of singlet-triplet perturbations, and implications for intersystem crossing in the isolated molecule, J. Chem. Phys. 89, 1813.

Koshi, M., Y.P. Vlahoyannis and R.J. Gordon, 1987, Vibrational relaxation of highly excited molecules: mode specific vibrational energy transfer from $\mathrm{SF}_{6}$ to $\mathrm{N}_{2} \mathrm{O}$, J. Chem. Phys. 86, 1311.

Koszykowski, M.L., D.W. Noid, M. Tabor and R.A. Marcus, 1981, On the use of correlation functions and the onset of chaotic motion, J. Chem. Phys. 74, 2530.

Koszykowski, M.L., D.W. Noid and R.A. Marcus, 1982a, Semiclassical theory of intensities of vibrational fundamentals, overtones and combination bands, J. Chem. Phys. 86, 2113.

Koszykowski, M.L., W.-K. Liu and D.W. Noid, 1982b, Effects of collisional relaxation on spectral functions in the quasi-periodic and chaotic regimes, J. Chem. Phys. 77, 2836.

Kryloff, N. and N. Bogoliuboff, 1947, Introduction to Nonlinear Mechanics (Princeton Univ. Press, Princeton).

Kulp, T., R.S. Ruoff and J.D. McDonald, 1985, Limits on the lifetimes of intramolecular vibrational relaxation, J. Chem. Phys. 82, 2175.

Kummer, M. and R. Gompa, 1988, Nonlinearly coupled oscillators in quantum mechanics: A normal form approach, J. Math. Phys. 29. 1405.

Kuzmin, M.V. and A.A. Stuchebrukhov, 1985, Threshold energy dependence of intramolecular vibrational redistribution (IVR) rates of isolated polyatomic molecules, Chem. Phys. Lett. 119, 556.

Kuzmin, M.V., V.S. Letokhov and A.A. Stuchebryukhov, 1986a, Threshold energy dependence of the intramolecular vibrational relaxation rate for an isolated polyatomic molecule, Sov. Phys. - JETP 63, 264.

Kuzmin, M.V., I.V. Nemov, A.A. Stuchebrukhov, V.N. Bagratashvili and V.S. Letokhov, 1986b, Chaotic non-ergodic vibrational motion in a polyatomic molecule, Chem. Phys. Lett. 124, 522.

Kuzmin, M.V., V.S. Letokhov and A.A. Stuchebrukhov, 1987a, Intramolecular vibrational relaxation and IR absorption spectrum of vibrationally excited polyatomics. I., Comments At. Mol. Phys. 20, 127.

Kuzmin, M.V., V.S. Letokhov and A.A. Stuchebrukhov, 1987b, Intramolecular vibrational redistribution and IR absorption spectrum of vibrationally excited polyatomics. II., Comments At. Mol. Phys. 20, 139.

Lahmani, F., A. Tramer and C. Tric, 1974, Nonexponential decays in single vibronic level fluorescence: a comparison between kinetics and quantum mechanical treatment, J. Chem. Phys. 60, 443.

Laubereau, A., G. Wochner and W. Kaiser, 1976, Collective beating of molecular vibrations in liquids on the picosecond time scale, Opt. Commun. 17,91 . 
Lawrance, W.D. and A.E.W. Knight, 1983, Collisional deactivation of a selected energy level in $\mathrm{S}_{0}$ p-difluorobenzene embedded in a dense vibrational field: absolute rate constants for a variety of collision partners, J. Chem. Phys. 79, 6030 .

Lawrance, W.D. and A.E.W. Knight, 1985, Direct deconvolution of extensively perturbed spectra: the singlet-triplet molecular eigenstate spectrum of pyrazine, J. Phys. Chem. 89, 917.

Lawrance, W.D. and A.E.W. Knight, 1988, Rotational mechanisms in intramolecular vibrational energy redistribution: an examination of centrifugal and Coriolis coupling and the states contributing to time dynamical measurements, J. Phys. Chem. 92, 5900.

Lawton, R.T. and M.S. Child, 1979, Local mode vibrations of water, Mol. Phys. 37, 1799.

Lawton, R.T. and M.S. Child, 1981, Local and normal stretching vibrational states of $\mathrm{H}_{2} \mathrm{O}$, classical and semiclassical considerations, Mol. Phys. $44,709$.

Lax, M., 1964, Quantum relaxation, the shape of lattice absorption and inelastic neutron scattering lines, J. Phys. Chem. Sol. 25 , 485.

Lederman, S.M. and R.A. Marcus, 1988, The use of artificial intelligence methods in studying quantum intramolecular vibrational dynamics, J. Chem. Phys. 88, 6312.

Lederman, S.M., J.H. Runnels and R.A. Marcus, 1983, Densities of vibrational states of given symmetry species and their use in statistical estimates involving Coriolis and anharmonic effects, J. Phys. Chem. 87, 4364

Lederman, S.M., V. Lopez, G.A. Voth and R.A. Marcus, 1986. Quantum and classical energy transfer between ligands of a heavy metal atom, Chem. Phys. Lett. 124, 93.

Lederman, S.M., S.J. Klippenstein and R.A. Marcus, 1988, Application of artificial intelligence methods to intramolecular dynamics calculations, Chem. Phys. Lett. 146, 7.

Lederman, S.M., V. Lopez, V. Fairen, G.A. Voth and R.A. Marcus, 1989, Vibrational energy redistribution across a heavy atom, Chem. Phys. 139, 171.

Lehmann, K.K., 1983, On the relationship of normal modes to local modes in the vibrations of $D_{6 \mathrm{~h}}$ symmetry molecules, J. Chem. Phys. 79,1098 .

Letokhov, V.S. and A.A. Makarov, 1981, Polyatomic molecules in a strong infrared field, Sov. Phys. - Usp. 24, 366.

Levine, R.D. and J.L. Kinsey, 1986, Anharmonic local mode-normal mode transformations: an algebraic treatment, J. Phys. Chem. $90,3653$.

Levy, D.H., 1980, Laser spectroscopy of cold gas-phase molecules, Annu. Rev. Phys. Chem. 31, 197.

Lichtenberg, A.J. and M.A. Lieberman, 1983, Regular and Stochastic Motion (Springer, New York).

Lichtenberg, A.J. and M.A. Lieberman, 1988, Diffusion in two-dimensional mappings, Physica D 33, 211.

Lichtenberg, A.J. and B.P. Wood, 1989a, Diffusion on two-space and time scales, Phys. Rev. Lett. 62, 2213.

Lichtenberg, A.J. and B.P. Wood, 1989b, Diffusion through a stochastic web, Phys. Rev. A 39, 2153.

Lim, K.F. and R.G. Gilbert, 1986, The a priori calculation of collisional energy transfer in highly vibrationally excited molecules: the biased random walk model, J. Chem. Phys. 84, 6129.

Lin, C.S., 1988, Coriolis and anharmonicity couplings in the intramolecular vibrational energy flow: $\mathrm{H}_{3}^{+}$potential, J. Chem. Phys. 89,7485 .

Lin, S.H., A. Boeglin, Y. Fujimura and B. Fain, 1988, Theory of vibrational predissociation spectroscopy of weak complexes, Chem. Phys. Lett. $145,334$.

Livi, R., M. Pettini, S. Ruffo and A. Vulpiani, 1985, Further results on the equipartition threshold in large nonlinear Hamiltonian systems, Phys. Rev. A 31, 2740

Lombardi, M., P. Labastie, M.C. Bordas and M. Broyer, 1988, Is there a signature of the chaoticity of the associated classical system in the spectrum of the quantum system?, Ber. Bunsenges. Phys. Chem. 92, 387.

Lopez, V. and R.A. Marcus, 1982, Heavy mass barrier to intramolecular energy transfer, Chem. Phys. Lett. 93, 232.

Lopez, V., V. Fairen, S.M. Lederman and R.A. Marcus, 1986, Local group modes and the dynamics of intramolecular energy transfer across a heavy atom, J. Chem. Phys. 84, 5494.

Lopez, V., A. Suarez and V. Fairen, 1988, Quantum dynamics by classical averages revisited in: Synergetics, Order and Chaos, ed. M.G. Velarde (World Scientific, Singapore).

Lu, D.-H. and W.L. Hase, 1988, Classical trajectory calculation of the benzene overtone spectra, J. Phys. Chem. $92,3217$.

Lu, D.-H., W.L. Hase and R.J. Wolf, 1986, The sensitivity of IVR in benzene to bend-stretch potential energy coupling, J. Chem. Phys. 85, 4422.

Lunsford, G.H. and J. Ford, 1972, On the stability of periodic orbits for nonlinear oscillator systems in regions exhibiting stochastic behavior, J. Math. Phys. 13, 700.

Luo, X., P.T. Rieger, D.S. Perry and T.R. Rizzo, 1988, A new technique for state-to-state studies of unimolecular reactions, J. Chem. Phys. 89, 4448

Lupo, D.W. and M. Quack, 1987, IR-Laser Photochemistry, Chem. Rev. 87, 181.

MacKay, R.S., 1983, A renormalisation approach to invariant circles in area-preserving maps, Physica D 7, 283.

MacKay, R.S., J.D. Meiss and I.C. Percival, 1984, Transport in Hamiltonian systems, Physica D 13, 55.

Makarov, A.A., 1987, Microwave spectroscopy as a method of investigating the randomization of vibrational motion in a polyatomic molecule, Opt. Spectrosc. $62,697$.

Makarov, A.A. and V.V. Tyakht, 1982, Spectra of infrared transitions between highly excited states of polyatomic molecules, Sov. Phys. - JETP 56, 274.

Makarov, A.A. and V.V. Tyakht, 1987, Purely phase intramolecular relaxation and its effect on the spectra of the transitions between the highly excited molecular vibrational states, Sov. Phys. - JETP 66, 9.

Makri, N. and W.H. Miller, 1987, Basis set methods for describing the quantum mechanics of a "system" interacting with a harmonic bath, J. Chem. Phys. 86, 1451. 
Marcus, R.A., 1952, Unimolecular dissociations and free radical recombination reactions, J. Chem. Phys. $20,359$.

Marcus, R.A., 1983, On the theory of intramolecular energy transfer, Faraday Disc. Chem. Soc. 75, 103.

Marcus, R.A., 1988, Intramolecular dynamics and unimolecular reactions, Ber. Bunsenges. Phys. Chem. 92, 209.

Marcus, R.A., W.L. Hase and K.N. Swamy, 1984, RRKM and non-RRKM behavior in chemical activation and related studies, J. Phys. Chem. 88, 6717.

Marshall, K.T. and J.S. Hutchinson, 1987, Classical and quantum dynamics of OH overtone excitations in a model of propargyl alcohol, J. Phys. Chem. 91, 3219.

Martens, C.C. and G.S. Ezra, 1987a, Classical and semiclassical mechanics of strongly resonant systems: a Fourier transform approach, J. Chem. Phys. 86, 279.

Martens, C.C. and G.S. Ezra, 1987b, Classical, quantum mechanical, and semiclassical representations of resonant dynamics: a unified treatment, J. Chem. Phys. 87, 284.

Martens, C.C., M.J. Davis and G.S. Ezra, 1987, Local frequency analysis of chaotic motion in multidimensional systems: energy transport and bottlenecks in planar OCS, Chem. Phys. Lett. 142, 519.

Matsumoto, Y., L.H. Spangler and D.W. Pratt, 1983, ${ }^{1}$ B $_{3 u}$ Pyrazine. Experimental tests of the theory of radiationless transitions, Laser Chem. $2,91$.

Mazur, P. and E. Montroll, 1960, Poincaré cycles, ergodicity, and irreversibility in assemblies of coupled harmonic oscillators, J. Math. Phys. 1, 70.

McClelland, G.M., G.M. Nathanson, J.H. Frederick and F.W. Farley, 1988, Intramolecular vibration-rotation energy transfer and the orientational dynamics of molecules, Excited States 7, 83 .

Mcllroy, A. and D.J. Nesbitt, 1989, High resolution, slit jet infrared spectroscopy of hydrocarbons: quantum state specific mode mixing in CH stretch excited propyne, J. Chem. Phys. 91, 104.

McLennan, J.A., 1959, Poincaré recurrence times, Phys. Fluids 2, 92.

McLachlan, N.W., 1964, Theory and Application of Mathieu Functions (Dover Publ., New York).

Meiss, J.D. and E. Ott, 1986, Markov tree model of transport in area-preserving maps, Physica D 20, 387.

Meiss, J.D., J.R. Cary, C. Grebogi, J.D. Crawford and A. Kaufman, 1983, Correlations of periodic, area-preserving maps, Physica D 6, 375.

Micklavc, A., 1987, Quantum theory of energy exchange in direct encounters of polyatomic molecules with nonrigid surfaces, Surf. Sci. $180,379$.

Miller, R.E., 1986, Infrared laser photodissociation and spectroscopy of van der Waals molecules, J. Phys. Chem. 90, 3301.

Miller, W.H., 1987, Tunneling and state specificity in unimolecular reactions, Chem. Rev. 87, 19.

Miller, W.H., W.L. Hase and C.L. Darling, 1989, A simple model for correcting the zero-point energy problem in classical trajectory simulations of polyatomic molecules, J. Chem. Phys. 91, 2863.

Milonni, P.W., J.R. Ackerhalt, H.W. Galbraith and M.L. Shih, 1983, Exponential decay, recurrences, and quantum-mechanical spreading in a quasicontinuum model, Phys. Rev. A 28, 32.

Minton, T.K. and J.D. McDonald, 1988, Infrared laser induced fluorescence studies of state mixing in ground electronic state molecules. Ber. Bunsenges. Phys. Chem. 92, 350.

Moiseyev, N., 1983, Motion of wave packets in regular and chaotic systems, J. Chem. Phys. 79, 5945.

Moiseyev, N. and R. Bar-Adon, 1984, Vibrational predissociation resonances for a model $\mathrm{H}-\mathrm{C}-\mathrm{C} \rightarrow \mathrm{H}+\mathrm{C}=\mathrm{C}$ Hamiltonian by the complex coordinate method, J. Chem. Phys. 80, 1917.

Moore, R., F.E. Doany, E.J. Heilweil and R.M. Hochstrasser, 1983, Energy redistribution in large molecules. Direct study of intramolecular relaxation in the gas phase with picosecond gating, Faraday Disc. Chem. Soc. 75, 331.

Mori, H., 1965, Transport, collective motion, and Brownian motion, Prog. Theor. Phys. 33, 423.

Moser, J., 1959, New aspects in the theory of stability of Hamiltonian systems, Math. Rev. 20, 675.

Moser, J., 1962, Nachr. Akad. Wiss. Gottingen, II Math. Physik. Kl. 1.

Muhlbach, J. and J.R. Huber, 1986, Intramolecular dephasing and relaxation in the molecule butynal, J. Chem. Phys. 85, 4411.

Mukamel, S., 1978, On the nature of intramolecular dephasing processes in polyatomic molecules, Chem. Phys. $31,327$.

Mukamel, S., 1981, Reduced equations of motion for collisionless molecular multiphoton processes, Adv. Chem. Phys. 47 (Part 1), 509.

Mukamel, S., 1985, Direct versus redistributed fluorescence of supercooled molecules: the onset of intramolecular vibrational redistribution. J. Chem. Phys. 82, 2867.

Mukamel, S. and R. Islampour, 1984, A pure-dephasing model for overtone lineshapes, Chem. Phys. Lett. 108, 161.

Mukamel, S. and J. Jortner, 1977, Time evolution of excited molecular states, Excited States 3, 58.

Mukamel, S. and A. Nitzan, 1977, Resonance Raman scattering from a multilevel, thermally relaxing system, J. Chem. Phys. 66, 2462.

Mukamel, S. and R.E. Smalley, 1980, Fluorescence of supercooled molecules as a probe for intramolecular vibrational redistribution rates. J. Chem. Phys. 73, 4156.

Muller, D.J., R.I. McKay, G.B. Edwards, W.D. Lawrance, J.P. Hardy, A.B. Rock, K.J. Selway, S.H. Kable and A.E.W. Knight, 1988. Vibrational relaxation induced by very low energy collisions in the $S_{1}\left({ }^{1} B_{2 u}\right)$ state of naphtalene: a search for resonance enhancement of the cross section, $J$. Phys. Chem. 92, 3751.

Muller, R.P., J.S. Hutchinson and T.A. Holme, 1988, A model for impulsive mode-mode energy transfer in highly vibrationally excited molecules, J. Chem. Phys. 90, 4582 .

Nadler, W. and R.A. Marcus, 1987, Low-frequency expansion for probability amplitudes: an alternative approach to certain intramolecular dynamics problems, J. Chem. Phys. 86, 6982.

Nalewajski, R.F. and R.E. Wyatt, 1983, Collisional perturbation of regular and irregular intramolecular dynamics: A classical dynamical study, Chem. Phys. 81, 357. 
Nalewajski, R.F. and R.E. Wyatt, 1984, Collisional perturbation of regular and irregular intramolecular dynamics: a model quantum-mechanical study, Chem. Phys. 85, 117.

Narducci, L.M., S.S. Mitra, R.A. Shatas and C.A. Coulter, 1977, Selective multiple-photon absorption by an anharmonic molecule, Phys. Rev. A $16,247$.

Nathanson, G.M. and G.M. McClelland, 1984, Fluorescence polarization as a probe of the rotational dynamics of isolated highly excited molecules, J. Chem. Phys. $81,629$.

Nesbitt, D.J. and S.R. Leone, 1982, Infrared fluoresence studies of intramolecular vibrational relaxation in $\mathrm{C}_{1}-\mathrm{C}_{4}$ hydrocarbons following pulsed laser excitation of the first $\mathrm{CH}$ stretch overtone, Chem. Phys. Lett. 87, 123.

Neusser, H.J., U. Schubert, E. Riedle, A. Kiermeier, H. Kuhlewind and E.W. Schlag, 1988, Rotationally resolved intramolecular processes in benzene, Ber. Bunsenges. Phys. Chem. 92, 322.

Nitzan, A. and B.N.J. Persson, 1985, Vibrational dephasing by the exchange mechanism: some new results, J. Chem. Phys. 83, 5610.

Nitzan, A., J. Jortner and P. Rentzepis, 1971, Internal conversion in large molecules, Mol. Phys. $22,585$.

Nitzan, A., J. Jortner and P. Rentzepis, 1972, Intermediate level structure in highly excited electronic states of large molecules, Proc. R. Soc. London Ser. A 327, 367.

Noid, D.W. and S.K. Gray, 1988, Calculations of semiclassical transition frequencies from short-time classical trajectories, Chem. Phys. Lett. 145, 9.

Noid, D.W. and M.L. Koszykowski, 1980, Semiclassical calculation of compound state resonances, Chem. Phys. Lett. 73, 114.

Noid, D.W. and R.A. Marcus, 1975, Semiclassical calculation of bound states in a multidimensional system. Use of Poincaré's surface of section, J. Chem. Phys. 62, 2119.

Noid, D.W. and R.A. Marcus, 1977, Semiclassical calculation of bound states in a multidimensional system for nearly $1: 1$ degenerate systems, J. Chem. Phys. 67, 559.

Noid, D.W., M.L. Koszykowski and R.A. Marcus, 1977, A spectral analysis method of obtaining molecular spectra from classical trajectories, J. Chem. Phys. 67, 404.

Noid, D.W., M.L. Koszykowski and R.A. Marcus, 1979, Semiclassical calculation of bound states in multidimensional systems with Fermi resonance, J. Chem. Phys. 71, 2864.

Noid, D.W., M.L. Koszykowski and R.A. Marcus, 1980, Calculations related to quantum stochasticity, an example of overlapping avoided crossings, Chem. Phys. Lett. 73, 269.

Noid, D.W., M.L. Koszykowski and R.A. Marcus, 1981a, Quasiperiodic and stochastic behavior in molecules, Annu. Rev. Phys. Chem. $32,267$.

Noid, D.W., M.L. Koszykowski and R.A. Marcus, 1981b, Intramolecular dynamics in the quasiperiodic and stochastic regimes, in: Quantum Mechanics in Mathematics, Chemistry, and Physics, eds K.E. Gustafson and W.P. Reinhardt (Plenum, New York) p. 133.

Noid, D.W., M.L. Koszykowski and R.A. Marcus, 1983, Comparison of quantal calculation and semiclassical behavior at an isolated avoided crossing, J. Chem. Phys. 78, 4018.

NoorBatcha, I., L.M. Raff, D.L. Thompson and R. Viswanathan, 1986, Dynamics of unimolecular dissociation of silylene, J. Chem. Phys. 84, 4341.

Nordholm, K.S.J. and R. Zwanzig, 1975, A systematic derivation of exact generalized Brownian motion theory, J. Stat. Phys. $13,347$.

O'Connor, P.W. and E.J. Heller, 1988, Quantum localization for a strongly classically chaotic system, Phys. Rev. Lett. 61, 2288.

Ohta, N., O. Sekuguchi and H. Baba, 1987, Rotational effect on intramolecular vibrational redistribution in $\mathrm{S}_{1}$ of p-difluoro benzene vapor, J. Phys. Chem. 91, 2076.

Okajima, S., H. Saigusa and E.C. Lim, 1982, Quantum beats in the fluorescence of jet-cooled diazabenzenes, J. Chem. Phys. 76, 2096.

Oref, 'I. and B.S. Rabinovitch, 1979, Do highly excited reactive polyatomic molecules behave ergodically?, Acc. Chem. Res. $12,166$.

Orr, B.J. and I.W.M. Smith, 1987, Collision-induced vibrational energy transfer in small polyatomic molecules, J. Stat. Chem. 91, 6106.

Oxtoby, D.W. and S.A. Rice, 1976, Nonlinear resonance and stochasticity in intramolecular energy exchange, J. Chem. Phys. 65 , 1676.

Ozorio de Almeida, A.M., 1984a, Semiclassical matrix elements, Rev. Bras. Fis. 14, 62.

Ozorio de Almeida, A.M., 1984b, Tunneling and the semiclassical spectrum for an isolated nonlinear resonance, J. Phys. Chem. 86, 6139.

Page, R.H., Y.R. Shen and Y.T. Lee, 1987, Highly resolved spectra of local modes of benzene, Phys. Rev. Lett. 59, 1263.

Page, R.H., Y.R. Shen and Y.T. Lee, 1988, Local modes of benzene and benzene dimer, studied by infrared-ultraviolet double resonance in a supersonic beam, J. Chem. Phys. 88, 4621 .

Parmenter, C.S., 1982, Vibrational energy flow within excited electronic states of large molecules, J. Phys. Chem. 86, 1735.

Parmenter, C.S., 1983, Vibrational redistribution within excited states of polyatomic molecules, Faraday Disc. Chem. Soc. $75,7$.

Parris, P.E. and P. Phillips, 1988, The role of dephasing in some recent theories of quantum localization, J. Chem. Phys. 88, 3561.

Parris, P.E. and R. Silbey, 1987, Variational treatment of a harmonic oscillator coupled to a dissipative heat bath, J. Chem. Phys. $86,6381$.

Parson, R., 1988, Classical-quantum correspondence in vibrational energy relaxation of nonlinear systems, J. Chem. Phys. 89, 262.

Parson, R., 1989, Coriolis-Induced vibrational energy transfer in $\mathrm{D}_{2} \mathrm{CO}-\mathrm{D}_{2} \mathrm{CO}$ collisions: A classical perspective, J. Chem. Phys. 91, 2206.

Parson, R., 1990, Coriolis-induced vibrational energy transfer in $\mathrm{D}_{2} \mathrm{CO}-\mathrm{D}_{2} \mathrm{CO}$ collisions, II: A semiclassical sudden approximation, J. Chem. Phys. 92, 304.

Parson, R. and E.J. Heller, 1986a, Semiclassical mechanics of dissipative molecular processes: the correspondence principle and population relaxation, J. Chem. Phys. 85, 2569.

Parson, R. and E.J. Heller, 1986b, Classical and semiclassical aspects of dissipative molecular processes: radiative and nonradiative vibrational relaxation in polyatomic molecules, J. Chem. Phys. 85, 2581.

Pauli, W., 1928, in Probleme der Modernen Physik, ed. P. Debye (Hirzel, Leipzig) p. 30. 
Peierls, R., 1929, Zur kinetischen Theorie der Wärmeleitung in Kristallen, Ann. Phys. 3, 1055.

Percival, I.C., 1974, Variational principles for the invariant toroids of classical dynamics, J. Phys. A 7, 794.

Percival, I.C., 1979, Variational principles for invariant tori and cantori, in: Nonlinear Dynamics and the Beam-Beam Interaction, Proc. AIP Conf. No. 57, eds M. Month and J.C. Herrera (American Institute of Physics, New York) p. 302.

Peres, A., 1984, Stability of quantum motion in chaotic and regular systems, Phys. Rev. A 20, 1610.

Perry, J.W. and A.H. Zewail, 1984, High energy CH-overtone spectra of benzene at $1.8 \mathrm{~K}$, J. Chem. Phys. 80, 5333.

Persch, G., E. Mehdizadeh, W. Demtröder, Th. Zimmermann, H. Koppel and L.S. Cederbaum, 1988, Vibronic level density of excited $\mathrm{NO}_{2}$ states and its statistical analysis, Ber. Bunsenges. Phys. Chem. 92, 312.

Persson, B.N.J. and M. Persson, 1980, Vibrational lifetime for CO adsorbed on $\mathrm{Cu}(100)$, Solid State Commun. $36,175$.

Petrosky, T.Y., 1984, Chaos and irreversibility in a conservative nonlinear dynamical system with a few degrees of freedom, Phys. Rev. A $29,2078$.

Peyerimhoff, S., M. Lewerenz and M. Quack, 1984, Spectroscopy and dynamics of the isolated $\mathrm{CH}$-chromophore in $\mathrm{CD}_{3} \mathrm{H}$ : experiment and theory, Chem. Phys. Lett. 109, 563.

Pique, J.P., M. Lombardi, Y. Chen, R.W. Field and J.L. Kinsey, 1988, New order out of the chaotic bath of highly vibrational states of $\mathrm{C}_{2} \mathrm{H}_{2}$, Ber. Bunsenges. Phys. Chem. 92, 422.

Poincaré, H., 1890, Non-existance des integrales uniformes, Acta Math. 13, 259.

Poincaré, H., 1957, Méthodes Nouvelles de la Mécanique Celeste (English translation, Dover Publ., New York, 1957$)$ p. 233.

Pollak, E., 1985, Periodic orbits and the theory of reactive scattering, in: The Theory of Chemical Reaction Dynamics, Vol. III, ed. M. Baer (CRC Press, Boca Raton, Florida) p. 123.

Pollak, E. and M.S. Child, 1980, Classical transition state theory: a lower bound to the reaction probability, J. Chem. Phys. $72,1669$.

Pollak, E., M.S. Child and P. Pechukas, 1980, Classical transition state theory: A lower bound to the reaction probability, J. Chem. Phys. $72,1669$.

Prasad, M., 1981, Theory of intramolecular energy relaxation and vibrational overtone resonance absorption lineshapes of isolated polyatomic molecules, Physica B + C 106, 106.

Pritchard, H.O., 1984, The Quantum Theory of Unimolecular Reactions (Cambridge Univ. Press, New York).

Quack, M., 1978, Theory of unimolecular reactions induced by monochromatic infrared radiation, J. Chem. Phys. 69, 1282.

Quack, M., 1979a, Master equations for photochemistry with intense infrared light, Ber. Bunsenges. Phys. Chem. 83, 757.

Quack, M. 1979b, Quantitative comparison between detailed (state selected) relative rate data and averaged (thermal) absolute rate data for complex forming reactions, J. Phys. Chem. 83, 150.

Quack, M., 1981a, Statistical mechanics and dynamics of molecular fragmentation, Nuovo Cimento B 63, 358.

Quack, M., 1981b, On normal, local and global vibrational states, Faraday Disc. Chem. Soc. 71, 359.

Quack, M., 1982, Reaction dynamics and statistical mechanics of the preparation of highly excited states by intense infrared radiation, Adv. Chem. Phys. 50, 395.

Quack, M., 1983, Some kinetic and spectroscopic evidence on intramolecular energy relaxation processes in polyatomic molecules, in: Energy Storage and Redistribution in Molecules, ed. J. Hinze (Plenum, New York, 1983) p. 493.

Quack, M., 1985, On the densities and numbers of rovibronic states of a given symmetry species: rigid and nonrigid molecules, transition states, and scattering channels, J. Chem. Phys. 82, 3277.

Quack, M. and J. Troe, 1977, in: Gas Kinetics and Energy Transfer 2, 175, eds P.G. Ashmore and R.J. Donovan (The Chemical Society, London),

Quapp, W., 1989, An explanation of the direction of relaxation in the HCN gas microwave laser, and a new assignment of further laser lines along the path of internal vibrational energy redistribution, Appl. Phys. B 48, 257.

Quapp, W. and D. Heidrich, 1988, Interpretation of the IR spectral intensity anomaly of HCN by potential energy surface bifurcation along a normal mode, Infrared Phys. 28, 83.

Radons, G., T. Geisel and J. Rubner, 1989, Classical chaos versus quantum dynamics: KAM tori and cantori as dynamical barriers, Adv. Chem. Phys. 73, 891 .

Raff, L., 1989, Intramolecular energy transfer and mode-specific effects in unimolecular reactions of 1,2 difluoroethane, J. Chem. Phys. $90,6313$.

Raff, L.M. and D.L. Thompson, 1985, The classical trajectory approach to reactive scattering, in: Theory of Chemical Reaction Dynamics, Vol. III, ed. M. Baer (CRC Press, Boca Raton, FL) p. 1.

Raff, L., R. Viswanathan and D.L. Thompson, 1984, Unimolecular dissociation of methane: a trajectory study using Metropolis sampling, J. Chem. Phys. 80, 6141.

Rai, S.N. and K.G. Kay, 1984, Comparison of quantum, classical, and statistical behavior in dissociating triatomics, J. Chem. Phys. $80,4961$.

Rainbird, M.W., B.S. Webb and A.E.W. Knight, 1988, State-to-state vibrational relaxation in ${ }^{1} \mathrm{~B}_{2 \mathrm{u}}$ benzene- $\mathrm{d}_{6}$ induced by low energy collisions with $\mathrm{He}, \mathrm{H}_{2}, \mathrm{~N}_{2}$, and Ar, J. Chem. Phys. 88, 2416.

Ramachandran, B. and K.G. Kay, 1985, Semiclassical ergodic properties of the Hénon-Heiles system, J. Chem. Phys. 83, 6316.

Ramaswamy, R. and R.A. Marcus, 1981, Perturbative examination of avoided crossings, J. Chem. Phys. 74, 1379.

Ramaswamy, R., S. Augustin and H. Rabitz, 1978, Stochastic theory of intramolecular energy transfer, J. Chem. Phys. $69,5509$.

Ranfagni, A., D. Mugnai and R. Englman, 1984, Semiclassical treatments of non-radiative processes, Phys. Rep. $108,165$.

Rashev, S., 1985, On the role of intramolecular vibrational energy redistribution in infrared multiphoton excitation of polyatomic molecules, Phys. Lett. A $109,265$.

Rashev, S. and I. Kancheva, 1987, Intramolecular vibrational redistribution in the course of infrared multiphoton excitation, Rev. Roum. Phys. 32, 185. 
Ratner, M.A., R.B. Gerber and V. Buch, 1987, Excited vibrational states: semiciassical self-consistent-field and statistical considerations, in: Stochasticity and Intramolecular Redistribution of Energy, eds R. Lefebvre and S. Mukamel (Reidel, Dordrecht) p. 57.

Rechester, A.B. and T.H. Stix, 1979, Stochastic instability of a nonlinear oscillator, Phys. Rev. A 19, 1656.

Reddy, K.V., D.F. Heller and M.J. Berry, 1982, Highly vibrationally excited benzene: Overtone spectroscopy and intramolecular dynamics of $\mathrm{C}_{6} \mathrm{H}_{6}$, $\mathrm{C}_{6} \mathrm{D}_{6}$ and partially deuterated or substituted benzenes, J. Chem. Phys. 76, 2814.

Reinhardt, W.P., 1982, Chaotic dynamics, semiclassical quantization, and mode-mode energy transfer: the Boulder view, J. Phys. Chem. 86, 2158.

Reinhardt, W.P., 1989, Adiabatic switching: a tool for semiclassical quantization and a new probe of classically chaotic phase space, in: Lasers, Molecules, and Methods, eds J.O. Hirschfelder, R.E. Wyatt and R.D. Coalson (Wiley, New York) p. 925.

Reinhardt, W.P. and I. Dana, 1987, Semi-classical quantization, adiabatic invariants and classical chaos, Proc. R. Soc. London A $413,157$.

Reinhardt, W.P. and C. Duneczky, 1988, Is there chemically significant trapping of vibrational energy in localized and chemically identifiable moieties?, J. Chem. Soc. Faraday Trans. $284,1511$.

Reinhardt, W.P. and C. Jaffé, 1981, in: Quantum Mechanics in Mathematics, Chemistry and Physics, eds K.E. Gustavson and W.P. Reinhardt (Plenum, New York).

Reisler, H. and C. Wittig, 1986, Photo-initiated unimolecular reactions, Annu. Rev. Phys. Chem. 37, 307.

Rice, S.A., 1981, An overview of the dynamics of intramolecular transfer of vibrational energy, Adv. Chem. Phys. 47, 117.

Rice, S.A. and C. Cerjan, 1983, Very low energy collision induced vibrational relaxation: an overview, Laser Chem. 2, 137.

Rice, B.M., L.M. Raff and D.L. Thompson, 1986, Classical trajectory study of the unimolecular dissociation of ammonia, J. Chem. Phys. 85, 4392.

Riedle, E., H.J. Neusser, E.W. Schlag and S.H. Lin, 1984, Intramolecular vibrational relaxation in benzene, J. Phys. Chem. 88, 1988.

Rizzo, T.R., C.C. Hayden and F.F. Crim, 1984, State-resolved product detection in the overtone vibration initiated unimolecular decomposition of $\mathrm{HOOH}\left(6 \nu_{\mathrm{OH}}\right)$, J. Chem. Phys. 81, 4501.

Robinson, G.W., 1967, Intersystem crossing in gaseous molecules, J. Chem. Phys. 47, 1967.

Robinson, G.W. and R.P. Frosch, 1962, Theory of electronic energy relaxation in solid phase, J. Chem. Phys. $37,1962$.

Robinson, P.J. and K.A. Holbrook, 1972, Unimolecular Reactions (Wiley, New York).

Rock, A.B., C.M. van Zoeren, S.H. Kable, G.B. Edwards and A.E.W. Knight, 1988, Is there resonance enhancement of the cross section for vibrational relaxation induced by very low energy collisions? The $I_{2}-$ He system revisited, J. Chem. Phys. 89, 6777 .

Rogers, P.J., D.C. Montague, J.P. Frank S.C. Tyler and F.S. Rowland, 1982, Non-RRKM decomposition in a chemical activation system: thermal atomic fluorine plus tetrallyl tin, Chem. Phys. Lett. 89, 9.

Rogers, P.J., J.I. Selco and F.S. Rowland, 1983, Non-RRKM decomposition in a chemical activation system: reaction of fluorine atoms with tetrallyl germanium, Chem. Phys. Lett. 97, 313.

Rosenbluth, M.N., R.Z. Sagdeev, J.B. Taylor and G.M. Zaslavskii, 1966, Destruction of magnetic surfaces by magnetic field irregularities, Nucl. Fusion 6, 297.

Rosker, M.J., F.W. Wise and C.L. Tang, 1986, Femtosecond relaxation dynamics of large molecules, Phys. Rev. Lett. 57, 321.

Sagdeev, R.Z., D.A. Usikov and G.M. Zaslavsky, 1988, Nonlinear Physics - From the Pendulum to Turbulence and Chaos (Harwood, New York).

Sahm, D.K. and T. Uzer, 1989a, Intramolecular energy transfer induced by potential and Coriolis couplings, J. Chem. Phys. $90,3159$.

Sahm, D.K. and T. Uzer, 1989b, The geometry of vibration-induced rotational mixing in molecules, Chem. Phys. Lett. $163,5$.

Sahm, D.K., S.W. McWhorter and T. Uzer, 1989, Generalizing the Chirikov paradigm: a geometrical view, J. Chem. Phys. 91, 219.

Sazonov, V.N. and A.A. Stuchebrukhov, 1981, On statistics of the ensemble of oscillators under excitation. II. Parametric excitation of linear quantum oscillators, Chem. Phys. 56, 391.

Sazonov, V.N. and A.A. Stuchebryukhov, 1983, Excitation of a quantum linear oscillator in a thermostat with a nonmonochromatically varied frequency by an external force, Sov. Phys. - JETP 57, 1.

Scheck, I. and J. Jortner, 1979, Random coupling model for multiphoton photofragmentation of large molecules, J. Chem. Phys. $70,3016$.

Scheck, I. and R.E. Wyatt, 1987, Effect of diagonal and off-diagonal disorder in the multiphoton excitation of an active mode coupled to a bath: use of the irreducible Recursive Residue Generation Method, in: Stochasticity and Intramolecular Redistribution of Energy, eds R. Lefebvre and S. Mukamel (Reidel, Dordrecht) p. 95.

Scherer, N.F and A. H. Zewail, 1987, Picosecond photofragment spectroscopy II. The overtone-initiated unimolecular reaction $\mathrm{H}_{2} \mathrm{O}_{2}\left(\nu_{\mathrm{OH}}=\right.$ 5) $\rightarrow 2 \mathrm{OH}$, J. Chem. Phys. 87, 97.

Scherer, N.F., F.E. Doany, A.H. Zewail and J.W. Perry, 1986, Direct picosecond time resolution of unimolecular reactions initiated by local mode excitation, J. Chem. Phys. 84, 1932.

Schewe, P.F. and J. Gollub, 1985, Chaos: A Glossary (American Institute of Physics, New York).

Segall, J. and R.N. Zare, 1988, Overtone-induced isomerization of allyl isocyanide, J. Chem. Phys. 89, 5704.

Shapiro, M., J. Ronkin and P. Brumer, 1988, The conservation of the correlation length of quantum and classical chaotic states, Ber. Bunsenges. Phys. Chem. 92, 212.

Shenker, S.J. and L.P. Kadanoff, 1982, Critical behavior of a KAM surface. I. Empirical results, J. Stat. Phys. $27,631$.

Shi, S. and W.H. Miller, 1985, A semiclassical model for intramolecular vibrational relaxation of local mode overtones in polyatomic molecules, Theor. Chim. Acta $68,1$.

Shin, H.K., 1989, Intramolecular dynamics of energy flow between van der Waals bonds in a collisionally excited Ar-N=N-Ar, Chem. Phys. Lett. 156,536 . 
Shirts, R.B., 1986, Decoupling of the local mode stretching vibrations of water through rotational excitation. I. Quantum mechanics, J. Chem. Phys. 85, 4949.

Shirts, R.B., 1987a, The identification of normal mode behavior in the resonance picture of local mode dynamics: normal mode splittings, stability of periodic orbits and energy transfer rates for coupled Morse oscillators, Chem. Phys. 114, 187.

Shirts, R.B., 1987b, Rotational decoupling of vibrational modes due to rotational excitation: Application to a model of the HDO stretching motions, Int. J. Quantum Chem. 31, 119.

Shirts, R.B. and W.P. Reinhardt, 1982, Approximate constants of motion for classically chaotic vibrational dynamics: vague tori, semiclassical quantization, and classical intramolecular energy flow, J. Chem. Phys. 77, 5204.

Shuryak, E.V., 1976, Nonlinear resonance in quantum systems, Sov. Phys. - JETP 44, 1070.

Sibert III, E.L., 1988, Theoretical studies of vibrationally excited polyatomic molecules using canonical Van Vleck perturbation theory, J. Chem. Phys. 88, 4378.

Sibert III, E.L., 1989, Rotationally induced vibrational mixing in formaldehyde, J. Chem. Phys. 90, 2672.

Sibert III, E.L., W.P. Reinhardt and J.T. Hynes, 1982a, Classical dynamics of energy transfer between bonds in ABA triatomics, J. Chem. Phys. 77, 3583.

Sibert III, E.L., J.T. Hynes and W.P. Reinhardt, 1982b, Quantum mechanics of local mode ABA triatomic molecules, J. Chem. Phys. $77,3595$.

Sibert III, E.L., J.T. Hynes and W.P. Reinhardt, 1983, Fermi resonance from a curvilinear perspective, J. Phys. Chem. 87, 2032.

Sibert III, E.L., J.T. Hynes and W.P. Reinhardt, 1984a, Classical dynamics of highly excited CH and CD overtones in benzene and perdeuterobenzene, J. Chem. Phys. 81, 1135.

Sibert III, E.L., W.P. Reinhardt and J.T. Hynes, 1984b, Intramolecular vibrational relaxation and spectra of CH and CD overtones in benzene and perdeuterobenzene, J. Chem. Phys. 81, 1115.

Siebrand, W., W.L. Meerts and D.W. Pratt, 1989, Analysis and deconvolution of some $J^{\prime} \neq 0$ rovibronic transitions in the high resolution $S_{1} \leftarrow S_{0}$ fluorescence excitation spectrum of pyrazine, J. Chem. Phys. 90, 1313.

Simons, J.P., 1984, Photodissociation: a critical survey, J. Phys. Chem. 88, 1287.

Sinha, A., R.L. Van der Wal, L.J. Butler and F.F. Crim, 1987, Two-color vibrationally mediated photodissociation of nitric acid, J. Phys. Chem. 91, 4645.

Skodje, R.T., 1989, Uniform adiabatic invariance analysis of chemical reaction dynamics, J. Chem. Phys. 90, 6193.

Skodje, R.T. and J.R. Cary, 1988, An analysis of the adiabatic switching method: foundations and applications, Comput. Phys. Rep. 8, 221.

Skodje, R.T and M.J. Davis, 1988, A phase-space analysis of the collinear I + HI reaction, J. Chem. Phys. 88, 2429.

Skodje, R.T., D. Schwenke, D.G. Truhlar and B.C. Garrett, 1984a, Reaction-path Hamiltonian model of partial widths for vibrationally elastic and inelastic decay of adiabatically trapped reactive resonances, J. Phys. Chem. 88, 628.

Skodje, R.T., D. Schwenke, D.G. Truhlar and B.C. Garrett, 1984b, Calculation of partial widths and isotope effects for reactive resonances by a reaction-path Hamiltonian model: test against accurate quantal results for a twin saddle-point system, J. Chem. Phys. 80, 3569.

Skodje, R.T., H.W. Rohrs and J. VanBuskirk, 1989, Flux analysis, the correspondence principle, and the structure of quantum phase space, Phys. Rev. A 40, 2894.

Slater, N.B., 1959, Theory of Unimolecular Reactions (Cornell Univ. Press, Ithaca, NY).

Sloane, C.S. and W.L. Hase, 1977, On the dynamics of state-selected unimolecular reactions: chloroacetylene dissociation and predissociation, J. Chem. Phys. 66, 1523.

Smalley, R.E., 1982, Vibrational randomization measurements with supersonic beams, J. Phys. Chem. 86, 3504.

Smalley, R.E., D.H. Levy and L. Wharton, 1976, The fluorescence excitation spectrum of the HeI, van der Waals complex, J. Chem. Phys. 64, 3266.

Smith, D.D., S.A. Rice and W. Struve, 1983, Intramolecular dephasing. Picosecond evolution of wavepacket states in a molecule with intermediate-case level structure, Faraday Disc. Chem. Soc. 75, 173.

Smith, R.S., R.B. Shirts and C.W. Patterson, 1987, Intramolecular energy transfer in the HNC/HCN isomerization reaction: quasiclassical state specific isomerization rates controlled by localized potential features, J. Chem. Phys. 86, 4452.

Sorbie, K.S. and N.C. Handy, 1976, Semiclassical eigenvalues for non-separable bound systems from classical trajectories: the degenerate case, Mol. Phys. 32, 1327.

Spears Jr, L.G. and J.S. Hutchinson, 1988a, Classical dynamics of transdiimide: intramolecular vibrational relaxation involving an active torsion, J. Chem. Phys. 88, 240.

Spears Jr, L.G. and J.S. Hutchinson, 1988b, Classical dynamics of an internal Coriolis-induced isomerization of trans-diimide, J. Chem. Phys. 88, 250.

Stannard, P.R. and W.M. Gelbart, 1981, Intramolecular vibrational energy redistribution, J. Phys. Chem. 85, 3592.

Stechel, E.B. and E.J. Heller, 1984, Quantum ergodicity and spectral chaos, Annu. Rev. Phys. Chem. 35, 563.

Stephenson, J.C., D.S. King, M.F. Goodman and J. Stone, 1979, Experiment and theory for $\mathrm{CO}_{2}$ laser-induced $\mathrm{CF}_{2} \mathrm{HCl}$ decomposition rate dependence on pressure and intensity, J. Chem. Phys. 70, 4496.

Stewart, G.M. and J.D. McDonald, 1983, Intramolecular vibrational relaxation from CH stretch fundamentals, J. Chem. Phys. $78,3907$.

Stone, J., E. Thiele and M.F. Goodman, 1981, The restricted quantum exchange theory of intramolecular $T_{1}$ and $T_{2}$ relaxation rates, J. Chem. Phys. 75, 1712. 
Straub, J.E. and B.J. Berne, 1986, Energy diffusion in many-dimensional Markovian systems: the consequences of competition between inter- and intramolecular vibrational energy transfer, J. Chem. Phys. 85, 2999.

Stuchebryukhov, A.A., 1986, On the theory of intramolecular vibrational relaxation of polyatomic molecules, Sov. Phys. - JETP $64,1195$.

Stuchebrukhov, A.A., 1988, On the theory of intramolecular vibrational relaxation in highly excited polyatomic molecules: quasi-harmonic versus random-phase approximation, Chem. Phys. Lett. 145, 387.

Stuchebrukhov, A.A. and I.E. Khromov, 1987, IR absorption spectroscopy of highly vibrationally excited polyatomic molecules, Chem. Phys. Lett. $134,251$.

Stuchebrukhov, A.A., M.V. Kuzmin, V.N. Bagratashvili and V.S. Letokhov, 1986, Threshold energy dependence of intramolecular vibrational relaxation in polyatomic molecules, Chem. Phys. 107, 429.

Stuchebrukhov, A., S. Ionov and V. Letokhov, 1989, IR spectra of highly vibrationally excited large polyatomic molecules and intramolecular relaxation, J. Phys. Chem. 93, 5357.

Sumpter, B.G. and D.L. Thompson, 1985, Intramolecular vibrational energy flow in model four-atom systems, J. Chem. Phys. 82, 4557.

Sumpter, B.G. and D.L. Thompson, 1987a, Studies of the intramolecular dynamics of model polyatomic molecules, J. Chem. Phys. 86, 2805.

Sumpter, B.G. and D.L. Thompson, 1987b, Intramolecular vibrational relaxation from CH stretching modes in dimethylnitramine, J. Chem. Phys. 86,3301 .

Sumpter, B.G. and D.L. Thompson, 1987c, Intramolecular dynamics of the overtone induced isomerization of methyl isocyanide, J. Chem. Phys. $87,5809$.

Sumpter, B.G. and D.L. Thompson, 1988, Influence of rotation on the intramolecular dynamics of hydrogen peroxide, Chem. Phys. Lett. $153,243$.

Sumpter, B.G., C.C. Martens and G.S. Ezra, 1988, Interaction of molecular rotation with large-amplitude internal motions: a rigid twister model of hydrogen peroxide, J. Phys. Chem. 92, 7193.

Sundberg, R.L. and E.J. Heller, 1984, Predissociation rates as a probe of intramolecular dynamics, J. Chem. Phys. $80,3680$.

Swamy, K.N. and W.L. Hase, 1985, The heavy-atom effect in intramolecular energy transfer, J. Chem. Phys. 82, 123.

Swamy, K.N. and W.L. Hase, 1986, Quantum and semiclassical transitional frequencies for a Hamiltonian with stretch-bend potential energy coupling, J. Chem. Phys. 84, 361.

Swamy, K.N., W.L. Hase, B.C. Garrett, C.W. McCurdy and J.F. McNutt, 1986, Mode specificity in the model unimolecular reaction $\mathrm{H}-\mathrm{C}-\mathrm{C} \rightarrow \mathrm{H}+\mathrm{C}=\mathrm{C}, \mathrm{J}$. Phys. Chem. 90, 3517.

Swimm, R.T. and J.B. Delos, 1979, Semiclassical calculations of vibrational energy levels for nonseparable systems using the Birkhoff-Gustavson normal form, J. Chem. Phys. 71, 1706.

Swofford, R.L., M.E. Long and A.C. Albrecht, 1976, C-H vibrational states of benzene, napthalene, and anthracene in the visible region by thermal lensing spectroscopy and the local mode model, J. Chem. Phys. 65, 179.

Tabor, M., 1981, The onset of chaos in dynamical systems, Adv. Chem. Phys. 46, 73.

Tabor, M., 1989, Chaos and Integrability in Nonlinear Dynamics (Wiley, New York).

Tannor, D.J., S.A. Rice and P.M. Weber, 1985, Picosecond CARS as a probe of ground electronic state intramolecular vibrational distribution, J. Chem. Phys. 83, 6158.

Taylor, H.S. and T.P. Grozdanov, 1987, Semiclassical quantization by using the method of adiabatic switching of the perturbation , in: Stochasticity and Intramolecular Redistribution of Energy, eds R. Lefebvre and S. Mukamel (Reidel, Dordrecht) p. 81.

Taylor, R.D. and P. Brumer, 1983, Pulsed laser preparation and quantum superposition state evolution in regular and irregular systems, Faraday Disc. Chem. Soc. 75, 117.

Tennyson, J. and S.C. Farantos, 1984, Vibrational chaos in KCN: a comparison of quantum and classical calculations, Chem. Phys. Lett. 109 , 160.

Tennyson, J.L., M.A. Lieberman and A.J. Lichtenberg, 1980, Diffusion in near-integrable Hamiltonian systems with three degrees of freedom, in: Nonlinear Dynamics and the Beam-Beam Interaction, eds M. Month and J.C. Herrera, AIP Conf. Proc. 57 (Amer. Inst. Physics, New York) p. 272 .

Ter Haar, D., 1954, Elements of Statistical Mechanics (Reinhart and Company, New York) p. 358.

Tersigni, S.H. and S.A. Rice, 1988, How robust are bottlenecks to unimolecular fragmentation?, Ber. Bunsenges. Phys. Chem. $92,227$.

Thiele, E. and D.J. Wilson, 1961, Anharmonicity in unimolecular reactions, J. Chem. Phys. 35, 1256.

Thiele, E., M.F. Goodman and J. Stone, 1980a, Can lasers be used to break chemical bonds selectively? Opt. Eng. 19, 10.

Thiele, E., M.F. Goodman and J. Stone, 1980b, Restricted intramolecular vibrational relaxation in polyatomics and low selective effects, Chem. Phys. Lett. 69, 18

Ticich, T.M., T.R. Rizzo, H.-R. Dübal and F.F. Crim, 1986, Unimolecular reactions near threshold: the overtone vibration initiated decomposition of $\mathrm{HOOH}\left(5 v_{\mathrm{OH}}\right)$, J. Chem. Phys. 84, 1508.

Ticich, T.M., M.D. Likar, H.-R. Dübal, L.J. Butler and F.F. Crim, 1987, Vibrationally mediated photodissociation of hydrogen peroxide, J. Chem. Phys. 87,5820 .

Tomer, J.L., K.W. Holtzclaw, D.W. Pratt and L.H. Spangler, 1988, Phosphorescence excitation spectroscopy in supersonic jets. The lowest triplet state of pyrazine, J. Chem. Phys. 88, 1528.

Tric, C., 1976, Intramolecular vibrational redistribution following optical excitation, Chem. Phys. 14, 189.

Tromp, J.W. and W.H. Miller, 1986, New approach to quantum mechanical transition state theory, J. Phys. Chem. 90, 3470.

True, N.S., 1983, Effects of rapid intramolecular vibrational redistribution on molecular spectra at microwave frequencies, Chem. Phys. Lett. 101, 326. 
Truhlar, D.L., W.L. Hase and J.T. Hynes, 1983, Current status of transition-state theory, J. Phys. Chem. 87, 2664.

Tully, J.C., 1985, Summary abstract: the dynamics of energy flow at surfaces, J. Vac. Sci. Technol. A 3, 1664.

Uzer, T., 1984, Bend-stretch interactions in the presence of Fermi resonances: a semiclassical view, Chem. Phys. Lett. $110,356$.

Uzer, T., 1988, Dissociation dynamics of polyatomic molecules, Adv. At. Mol. Phys. 25, 417.

Uzer, T. and J.T. Hynes, 1985, A model study of overtone-induced isomerization: the role of nonlinear resonances, Chem. Phys. Lett. 113, 483.

Uzer, T. and J.T. Hynes, 1986, Intramolecular energy transfer in simple model organometallics, J. Phys. Chem. $90,3524$.

Uzer, T. and J.T. Hynes, 1987, Theoretical studies of overtone-induced chemical reactions, in: Stochasticity and Intramolecular Redistribution of Energy, eds R. Lefebvre and S. Mukamel (Reidel, Dordrecht) p. 273.

Uzer, T. and J.T. Hynes, 1989, Dissociation of remote bonds by overtone excitation: a model study of heavy-atom blocking, Chem. Phys. $139,163$.

Uzer, T. and R.A. Marcus, 1984, Quantization with operators appropriate to shapes of trajectories and classical perturbation theory, J. Chem. Phys. 81, 5013; erratum 84 (1985) 4724.

Uzer, T., D.W. Noid and R.A. Marcus, 1983, Uniform semiclassical theory of avoided crossings, J. Chem. Phys. 79, 4412.

Uzer, T., G.A. Natanson and J.T. Hynes, 1985a, Coriolis-induced intramolecular vibrational energy flow between anharmonic normal modes, Chem. Phys. Lett. 122, 12.

Uzer, T., J.T. Hynes and W.P. Reinhardt, 1985b, Overtone-induced dissociation of hydrogen peroxide: a classical trajectory study, Chem. Phys. Lett. 117,600 .

Uzer, T., W.P. Reinhardt and J.T. Hynes, 1986, Classical dynamics of energy flow and overtone-induced dissociation in $\mathrm{H}_{2} \mathrm{O}_{2}$ and $\mathrm{HDO}_{2}$, J. Chem. Phys. 85, 5791.

Uzer, T., B.D. MacDonald, Y. Guan and D.L. Thompson, 1988, Theoretical studies of mode-specificity in the dissociation of overtone-induced hydrogen peroxide, Chem. Phys. Lett. 152, 405.

Van der Meer, B.J., H.Th. Jonkman, G.M. ter Horst and J. Kommandeur, 1982a, Intermediate level structure, quantum beats, and nuclear spin effects in the electronic relaxation of the ${ }^{1} B_{3 u}\left(n \pi^{*}\right)$ state of pyrazine, J. Chem. Phys. 76, 2099.

Van der Meer, B.J., H.Th. Jonkman, J. Kommandeur, W.L. Meerts and W.A. Majewski, 1982b, Spectrum of the molecular eigenstates of pyrazine, Chem. Phys. Lett. 92, 565.

Van Hove, L., 1962, in: Fundamental Problems in Statistical Mechanics, ed. E.D.G. Cohen (North-Holland, Amsterdam) p. 157.

Van Roosmalen, O.S., F. Iachello, R.D. Levine and A.E.L. Dieperink, 1983, Algebraic approach to molecular rotation-vibration spectra. II. Triatomic molecules, J. Chem. Phys. 79, 2515.

Van Roosmalen, O.S., I. Benjamin and R.D. Levine, 1984, A unified algebraic model description for interacting vibrational modes in ABA molecules, J. Chem. Phys. 81, 5986.

Van Smaalen, S. and T.F. George, 1987, Pure dephasing of a vibrational adbond, J. Chem. Phys. 87, 7307.

Vivaldi, F., G. Casati and I. Guarneri, 1983, Origin of long-time tails in strongly chaotic systems, Phys. Rev. Lett. 51, 727.

Villalonga, E. and D.A. Micha, 1983, Vibrational energy transfer in atom-polyatomic collisions: the role of intramolecular dynamics in $\mathrm{Li}^{+}+\mathrm{CO}_{2}$ and $\mathrm{Li}^{+}+\mathrm{N}_{2} \mathrm{O}, \mathrm{J}$. Chem. Phys. 78, 3942.

Viswanathan, R., L.M. Raff and D.L. Thompson, 1984, Monte Carlo random walk calculations of unimolecular dissociation of methane, J. Chem. Phys. 81,3118 .

Viswanathan, R., L.M. Raff and D.L. Thompson, 1985, Monte Carlo transition-state study of angular momentum effects on the unimolecular dissociation of $\mathrm{CH}_{4}$ on the Duchovic-Hase-Schlegel ab initio surface, J. Chem. Phys. 82, 3083.

Volotikin, A.I., O.M. Braun and V.M. Yakovlev, 1986, Shift and broadening of adsorbate vibrational bonds, Surf. Sci. 172, 31.

Von Puttkamer, K., H.-R. Dübal and M. Quack, 1983, Time-dependent processes in polyatomic molecules during and after intense infrared irradiation, Faraday Disc. Chem. Soc. 75, 197.

Voth, G.A., 1986, On the relationship of classical resonances to the quantum mechanics of coupled oscillator systems, J. Phys. Chem. 90 , 3624.

Voth, G.A., 1987, Quasidissipative intramolecular dynamics: an adiabatically reduced coupled equations approach, J. Chem. Phys. 87, 5272.

Voth, G.A., 1988, An effective Golden Rule decay rate expression for quasidissipative IVR processes, J. Chem. Phys. 88, 5547.

Voth, G.A. and R.A. Marcus, 1985, Semiclassical theory of Fermi resonance between stretching and bending modes in polyatomic molecules, J. Chem. Phys. 82, 4064.

Voth, G.A. and R.A. Marcus, 1986, Adiabatically reduced coupled equations for intramolecular dynamics calculations, J. Chem. Phys. 84, 2254.

Voth, G.A., R.A. Marcus and A.H. Zewail, 1984, The highly excited C-H stretching states of $\mathrm{CHD}_{3}, \mathrm{CHT}_{3}$, and $\mathrm{CH}_{3} \mathrm{D}$, J. Chem. Phys. 81, 5495 .

Waite, B.A. and W.H. Miller, 1980, Model studies of mode specificity in unimolecular reaction dynamics, J. Chem. Phys. 73, 3713.

Waite, B.A. and W.H. Miller, 1981, Mode specificity in unimolecular reaction dynamics: the Hénon-Heiles potential energy surface, J. Chem. Phys. 74, 3910.

Waite, B.A. and W.H. Miller, 1982, A semiclassical multichannel branching model for describing state-specific unimolecular decomposition and other dynamical processes in polyatomic molecular systems, J. Chem. Phys. 76, 2412.

Walker, G.H. and J. Ford, 1969, Amplitude instability and ergodic behavior for conservative nonlinear systems, Phys. Rev, $188,416$.

Wardlaw, D.M. and R.A. Marcus, 1987, On the statistical theory of unimolecular processes, Adv. Chem. Phys. 70, 231.

Wardlaw, D.M., D.W. Noid and R.A. Marcus, 1984, Semiclassical and quantum vibrational intensities, J. Phys. Chem. $88,536$.

Watanabe, H., S. Tsuchiya and S. Koda, 1983, Rotation-dependent intramolecular processes of $\mathrm{SO}_{2}\left(\mathrm{~A}^{\prime} \mathrm{A}_{2}\right)$ in a supersonic jet, Faraday Disc. Chem. Soc. 75, 365

Weitz, E. and G. Flynn, 1981, Vibrational energy flow in the ground electronic states of polyatomic molecules, Adv. Chem. Phys. 47 (Part 2), 185. 
Whittaker, E.T., 1944, Analytical Dynamics (Dover Publ., New York) p. 432.

Wolf, R.J. and W.L. Hase, 1980a, Trajectory studies of model $\mathrm{H}-\mathrm{C}-\mathrm{C} \rightarrow \mathrm{H}+\mathrm{C}=\mathrm{C}$ dissociation. I. Random vibrational excitation, J. Chem. Phys. $72,316$.

Wolf, R.J. and W.L. Hase, 1980b, Quasiperiodic trajectories for a multidimensional anharmonic classical Hamiltonian excited above the unimolecular threshold, J. Chem. Phys. 73, 3779.

Wolf, R.J., D.S. Bhatia and W.L. Hase, 1986, Effect of bond stretch excitation on the attenuation of bending forces, Chem. Phys. Lett. $132,493$.

Wong, J.S., W.H. Green Jr, C.-K. Cheng and C.B. Moore, 1987, Coupling of CH stretching and bending vibrations in trihalomethanes, J. Chem. Phys. 86, 5994.

Wozny, C.E. and S.K. Gray, 1988, Intramolecular and fragmentation dynamics of van der Waals complexes, Ber. Bunsenges. Phys. Chem. 92, 236.

Wrigley, S.P. and B.S. Rabinovitch, 1983, Intramolecular vibrational relaxation in the 3-methyl-3-hexyl radical, Chem. Phys. Lett. 98, 386.

Wrigley, S.P., D.A. Oswald and B.S. Rabinovitch, 1984, On the question on heavy-atom blocking of intramolecular vibrational energy transfer, Chem. Phys. Lett. 104, 521.

Xiao, L. and M.E. Kellman, 1989, Unified semiclassical dynamics for molecular resonance spectra, J. Chem. Phys. 90, 6086.

Xie, B., 1988, Molecular spectra in chaotic regime expressed by the Wigner function. J. Chem. Phys. 89, 1351.

Zabusky, N.J. and M.D. Kruskal, 1965, Interaction of "solitons" in a collisionless plasma and the recurrence of initial states, Phys. Rev. Lett. 15, 240.

Zangwill, A., 1988, Physics at Surfaces (Cambridge Univ. Press, New York).

Zaslavsky, G.M., 1981, Stochasticity in Quantum Systems, Phys. Rep. 80, 157.

Zaslavsky, G.M., 1985, Chaos in Dynamical Systems (Harwood, New York).

Zaslavskii, G.M. and B.V. Chirikov, 1972, Stochastic instability of nonlinear oscillations, Sov. Phys. - Usp. 14, 549.

Zaslavskii, G.M. and N.N. Filonenko, 1968, Stochastic instability of trapped particles and conditions of applicability of the quasi-linear approximation, Sov. Phys. - JETP 25, 851.

Zewail, A.H., 1985, IVR: its coherent and incoherent dynamics, Ber. Bunsenges. Phys. Chem. 89, 264.

Zwanzig, R., 1960, Ensemble method in the theory of irreversibility, J. Chem. Phys. 33, 1338. 